Supporting Information for -

\title{
H-X Bond Activation via Hydrogen Transfer in Ruthenium N-Heterocyclic Carbene Complexes: Density Functional and Synthetic Studies
}

Sarah L. Chatwin, ${ }^{\dagger}$ Matthew G. Davidson, ${ }^{\dagger}$ Cheryl Doherty, ${ }^{\dagger}$ Steven M. Donald ${ }^{\dagger}$ Rodolphe F. R. Jazzar, ${ }^{\dagger}$ Stuart A. Macgregor, ${ }^{*}$ Garry McIntyre, ${ }^{\S}$ Mary F. Mahon ${ }^{\dagger}$ and Michael K. Whittlesey* ${ }^{\dagger}$

${ }^{\dagger}$ Department of Chemistry, University of Bath, Claverton Down, Bath BA2 7AY, UK, and

${ }^{\ddagger}$ School of Engineering and Physical Sciences, Heriot-Watt University, Edinburgh, EH14 4AS, UK, and ${ }^{\S}$ Institut Laue Langevin, BP156.38042 Grenoble, Cedex 9, France

\section{Contents:}

Computed Cartesian coordinates $(\AA ̊)$, energies and enthalpies (a.u.) for all stationary points including unique imaginary eigenvalues for all transition states. Enthalpies include a correction for zero-point energies and are adjusted to $298.15 \mathrm{~K}$.

Species are numbered according to the numbering scheme in the main text. 


\begin{tabular}{|lcll|}
\hline \multicolumn{4}{|l|}{ trans-2'-SH } \\
\multicolumn{3}{|l|}{ Energy $=-673.497862$} \\
Enthalpy $(0 \mathrm{~K})=-673.314439$ \\
\multicolumn{4}{|l}{ Enthalpy $(298.15 \mathrm{~K})=-673.297185$} \\
\multicolumn{4}{|l}{ Free Energy $=-673.360887$} \\
\hline Ru & 0.024146 & -0.012430 & 0.308078 \\
$\mathrm{H}$ & -1.676778 & 0.011524 & 0.445309 \\
$\mathrm{C}$ & -0.017821 & -0.027392 & -1.525894 \\
$\mathrm{O}$ & -0.043679 & -0.039871 & -2.709256 \\
$\mathrm{H}$ & 1.729864 & 0.005253 & 0.359046 \\
$\mathrm{C}$ & 0.027765 & 2.039231 & 0.336186 \\
$\mathrm{C}$ & 0.019227 & -2.072462 & 0.312927 \\
$\mathrm{~N}$ & -1.039567 & 2.908040 & 0.361910 \\
$\mathrm{C}$ & -0.656812 & 4.246285 & 0.318934 \\
$\mathrm{C}$ & 0.713404 & 4.245098 & 0.273784 \\
$\mathrm{~N}$ & 1.095922 & 2.906224 & 0.291520 \\
$\mathrm{~N}$ & 1.083842 & -2.941082 & 0.259572 \\
$\mathrm{C}$ & 0.696920 & -4.278200 & 0.238276 \\
$\mathrm{C}$ & -0.673359 & -4.274670 & 0.284857 \\
$\mathrm{~N}$ & -1.051024 & -2.935568 & 0.332092 \\
$\mathrm{H}$ & -1.986953 & 2.536621 & 0.360402 \\
$\mathrm{H}$ & 2.040825 & 2.533565 & 0.230519 \\
$\mathrm{H}$ & 2.031361 & -2.573007 & 0.216436 \\
$\mathrm{H}$ & -1.997370 & -2.562434 & 0.350660 \\
$\mathrm{H}$ & -1.365651 & 5.069833 & 0.325757 \\
$\mathrm{H}$ & 1.422551 & 5.067434 & 0.233689 \\
$\mathrm{H}$ & 1.403382 & -5.102600 & 0.195390 \\
$\mathrm{H}$ & -1.385359 & -5.095394 & 0.290429 \\
$\mathrm{~S}$ & 0.086132 & -0.150128 & 2.761578 \\
$\mathrm{H}$ & -0.873458 & 0.684120 & 3.266824 \\
$\mathrm{H}$ & 1.076756 & 0.677295 & 3.215824 \\
\hline
\end{tabular}




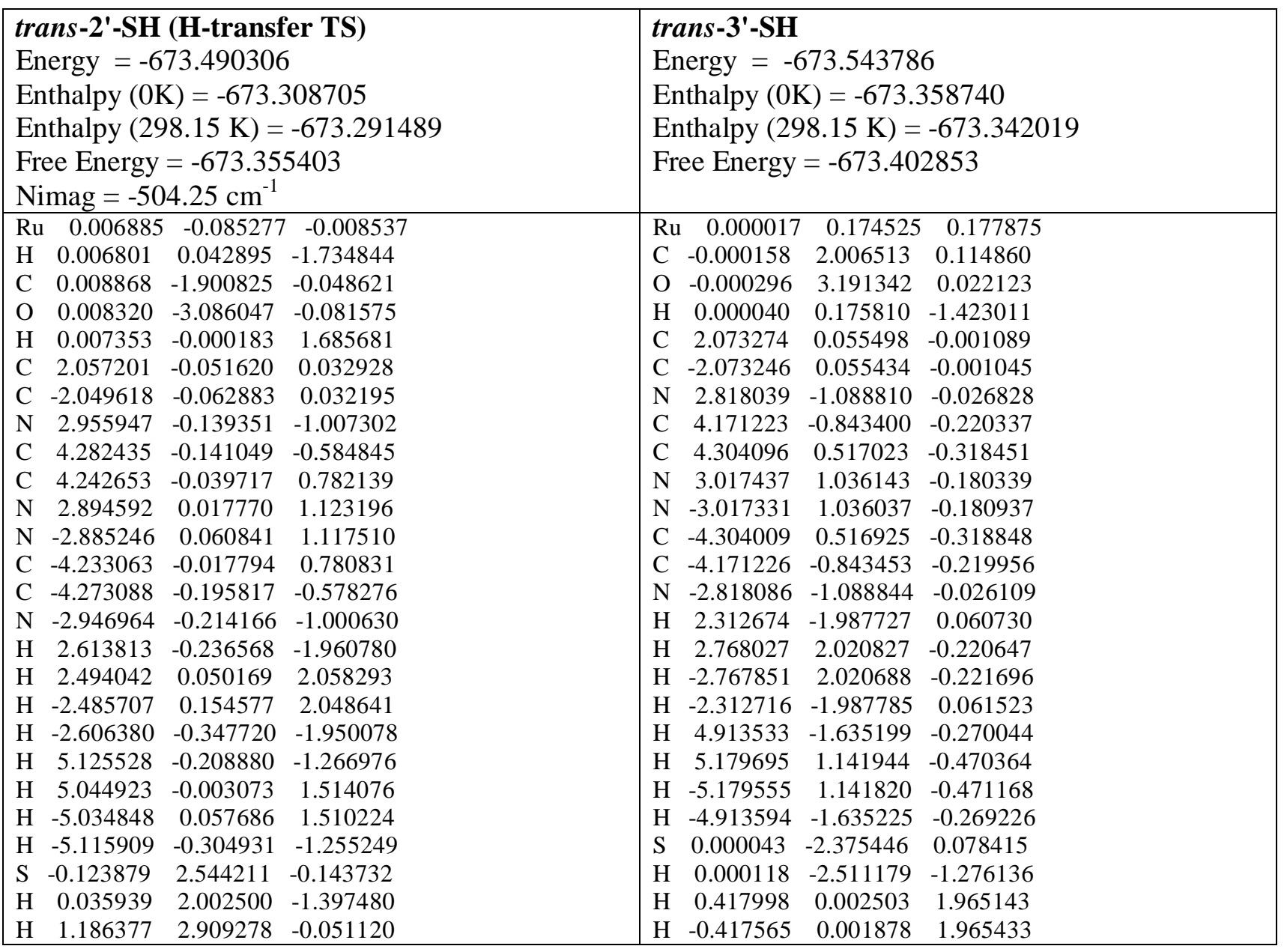




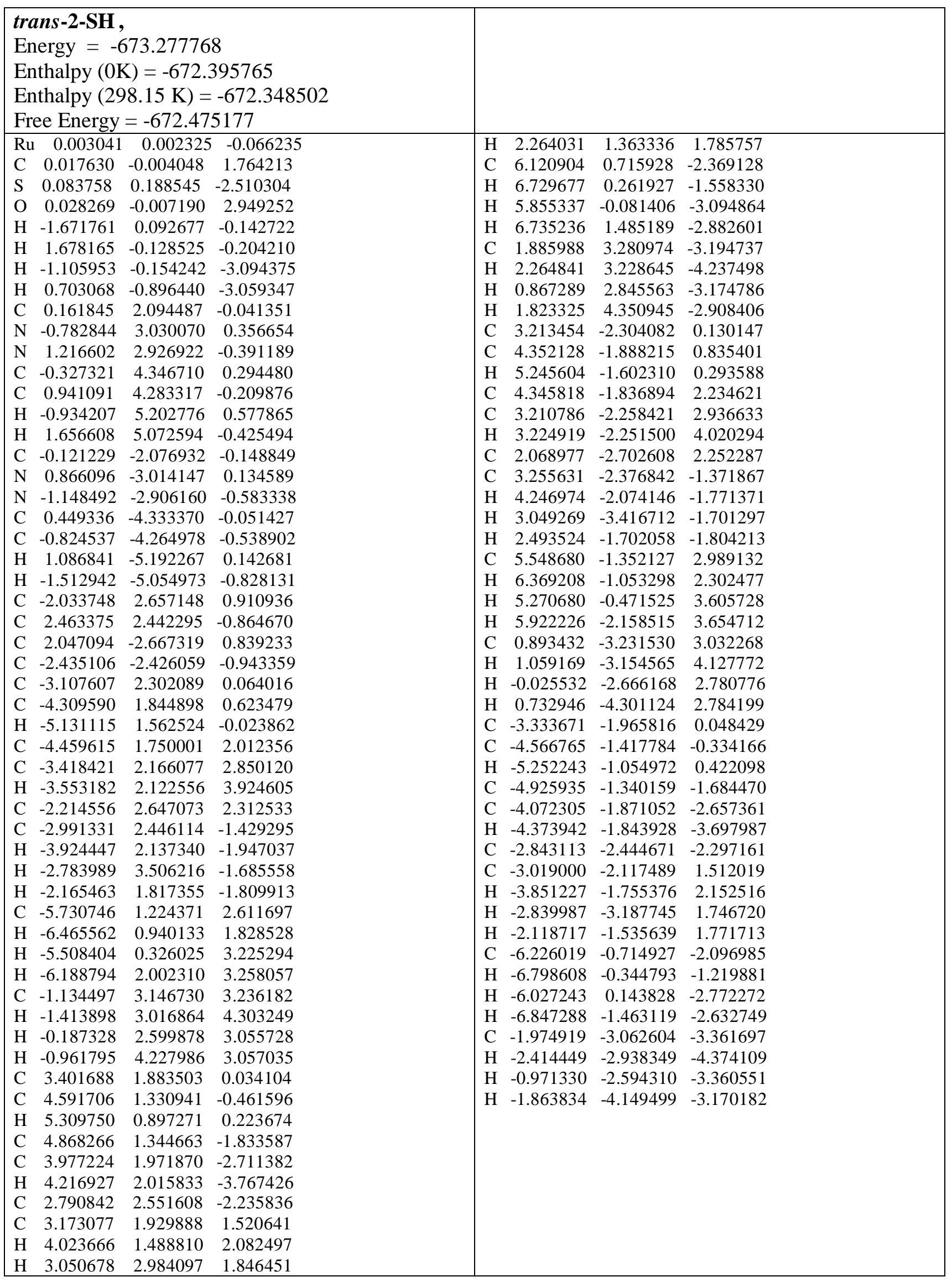




\begin{tabular}{|c|c|c|c|c|c|c|}
\hline \multicolumn{3}{|c|}{$\begin{array}{l}\text { trans-2-SH (H-transfer TS) } \\
\text { Energy }=-673.268757 \\
\text { Enthalpy }(0 \mathrm{~K})=-672.388558 \\
\text { Enthalpy }(298.15 \mathrm{~K})=-672.341389 \\
\text { Free Energy }=-672.467959 \\
\text { Nimag }=-522.48 \mathrm{~cm}^{-1}\end{array}$} & \multirow{2}{*}{\multicolumn{4}{|c|}{$\begin{array}{llll}\mathrm{H} & 2130459 & 1374650 & 1901065\end{array}$}} \\
\hline \multicolumn{3}{|c|}{\begin{tabular}{|cccc}
$\mathrm{Ru}$ & 0.001384 & 0.010481 & -0.096921
\end{tabular}} & & & & \\
\hline & -0.072509 & $-0.058081 \quad 1.712964$ & & 6.307657 & 0.721766 & -1.936262 \\
\hline & 0.081600 & $0.266018-2.717095$ & & 6.853343 & 0.276976 & -1.076912 \\
\hline & -0.094497 & $-0.102268 \quad 2.898838$ & $\mathrm{H}$ & 6.105892 & -0.081475 & -2.675858 \\
\hline & -1.689710 & $0.134990-0.361574$ & & 6.955850 & 1.492453 & -2.403904 \\
\hline & 1.663349 & $-0.109644 \quad-0.079243$ & & 2.150411 & 3.277205 & -3.106797 \\
\hline & -1.146670 & $-0.142152-2.252443$ & & 2.611078 & 3.220635 & -4.115872 \\
\hline & 0.476424 & $-0.964317-3.141185$ & & 1.130354 & 2.850621 & -3.167929 \\
\hline & 0.183697 & $2.097310-0.042412$ & $\mathrm{H}$ & 2.073182 & 4.348067 & -2.827774 \\
\hline & -0.775401 & $3.039621 \quad 0.301393$ & & 3.171253 & -2.304017 & -0.052810 \\
\hline & 1.252043 & $2.923382-0.366784$ & & 4.350354 & -1.940377 & 0.613484 \\
\hline & -0.314884 & $4.354228 \quad 0.242652$ & & 5.217812 & -1.632610 & 0.042240 \\
\hline & 0.972367 & $4.282326-0.210002$ & & 4.415885 & -1.961450 & 2.011602 \\
\hline & -0.931040 & $5.214152 \quad 0.492201$ & & 3.311462 & -2.403306 & 2.748474 \\
\hline & 1.696408 & $5.068496-0.407855$ & & 3.379323 & -2.447443 & 3.829242 \\
\hline & -0.146992 & $-2.070008-0.232023$ & & 2.131362 & -2.802360 & 2.102131 \\
\hline & 0.828053 & $-3.025604 \quad 0.030947$ & & 3.132584 & -2.281872 & -1.555980 \\
\hline & -1.200737 & $-2.883421 \quad-0.646928$ & $\mathrm{H}$ & 4.107668 & -1.972720 & -1.989087 \\
\hline & 0.381563 & $-4.336960-0.138842$ & $\mathrm{H}$ & 2.885088 & -3.293821 & -1.939645 \\
\hline & -0.902976 & $-4.247091-0.593141$ & & 2.365278 & -1.563452 & -1.899418 \\
\hline & 1.008298 & $-5.207247 \quad 0.039316$ & & 5.661420 & -1.527770 & 2.727013 \\
\hline & -1.611238 & $-5.025459-0.865615$ & & 6.451730 & -1.207358 & 2.015073 \\
\hline & -2.026693 & $2.672579 \quad 0.856792$ & & 5.426874 & -0.673571 & 3.396582 \\
\hline & 2.531295 & $2.433311 \quad-0.737959$ & & 6.055367 & -2.369185 & 3.334969 \\
\hline & 2.039647 & $-2.700726 \quad 0.694447$ & & 0.985842 & -3.342176 & 2.917972 \\
\hline & -2.508602 & $-2.394852-0.909805$ & & 1.205179 & -3.310059 & 4.006275 \\
\hline C & -3.117058 & $2.376183 \quad 0.010162$ & & 0.067899 & -2.750703 & 2.732972 \\
\hline & -4.319918 & $1.915908 \quad 0.565595$ & & 0.793541 & -4.398797 & 2.639628 \\
\hline & -5.154253 & $1.675690-0.081986$ & & -3.352699 & -1.987196 & 0.150273 \\
\hline C & -4.455530 & $1.768997 \quad 1.951895$ & & -4.599787 & -1.413277 & -0.138261 \\
\hline & -3.396003 & $2.130124 \quad 2.792367$ & & -5.242170 & -1.087078 & 0.670238 \\
\hline & -3.519411 & 2.048990 & & -5.033376 & -1.274128 & -1.461467 \\
\hline C & -2.189898 & $2.609426 \quad 2.258761$ & & -4.240773 & -1.768747 & -2.502292 \\
\hline C & -3.015981 & $2.589345-1.476046$ & & -4.602601 & -1.700516 & -3.521641 \\
\hline $\mathrm{H}$ & -3.955658 & $2.307622-1.997269$ & & -2.996474 & -2.360874 & -2.236608 \\
\hline & -2.808785 & $3.660082-1.683572$ & & -2.977949 & -2.244842 & 1.583670 \\
\hline $\mathrm{H}$ & -2.193636 & $1.980308 \quad-1.895546$ & & -3.770928 & -1.908157 & 2.284991 \\
\hline C & -5.731807 & $1.250900 \quad 2.546921$ & & -2.817019 & -3.332534 & 1.737126 \\
\hline $\mathrm{H}$ & -6.482914 & 1.015708 & & -2.052418 & -1.707097 & 1.839545 \\
\hline $\mathrm{H}$ & -5.524045 & 0.324152 & & -6.352217 & -0.629771 & -1.772797 \\
\hline $\mathrm{H}$ & -6.162843 & 2.013680 & $\mathrm{H}$ & -6.873011 & -0.296781 & -0.849737 \\
\hline C & -1.094710 & 3.068225 & $\mathrm{H}$ & -6.188349 & 0.256756 & -2.421052 \\
\hline $\mathrm{H}$ & -1.365204 & 2.909480 & & -7.005798 & -1.353314 & -2.303942 \\
\hline $\mathrm{H}$ & -0.155086 & $2.517862 \quad 2.979961$ & & -2.203501 & -2.954206 & -3.370216 \\
\hline $\mathrm{H}$ & -0.911217 & $4.152346 \quad 3.036258$ & $\mathrm{H}$ & -2.711501 & -2.810797 & -4.347467 \\
\hline C & 3.397776 & $1.883037 \quad 0.235820$ & & -1.205229 & -2.482653 & -3.428528 \\
\hline C & 4.626639 & $1.335424-0.160504$ & & -2.076938 & -4.044362 & -3.207602 \\
\hline $\mathrm{H}$ & 5.289330 & $0.907444 \quad 0.581926$ & & & & \\
\hline $\mathrm{C}$ & 5.012253 & $1.344682-1.505848$ & & & & \\
\hline C & 4.191979 & $1.964477 \quad-2.454690$ & & & & \\
\hline $\mathrm{H}$ & 4.516743 & $2.007621 \quad-3.487791$ & & & & \\
\hline $\mathrm{C}$ & 2.970055 & $2.542953 \quad-2.077813$ & & & & \\
\hline C & 3.055664 & $1.941119 \quad 1.700276$ & & & & \\
\hline $\mathrm{H}$ & 3.861658 & $1.507015 \quad 2.329505$ & & & & \\
\hline $\mathrm{H}$ & 2.906335 & $2.997687 \quad 2.006700$ & & & & \\
\hline
\end{tabular}




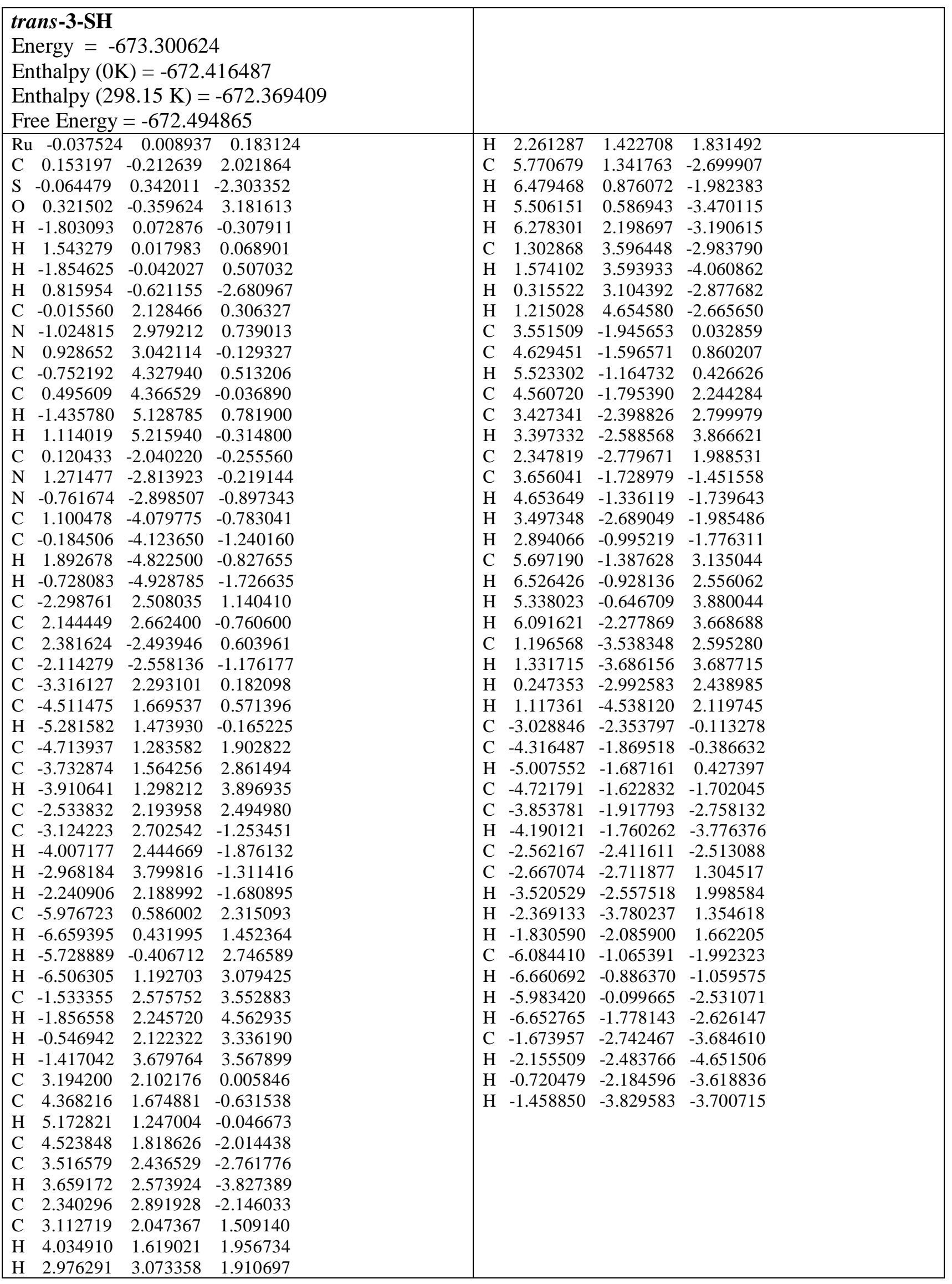




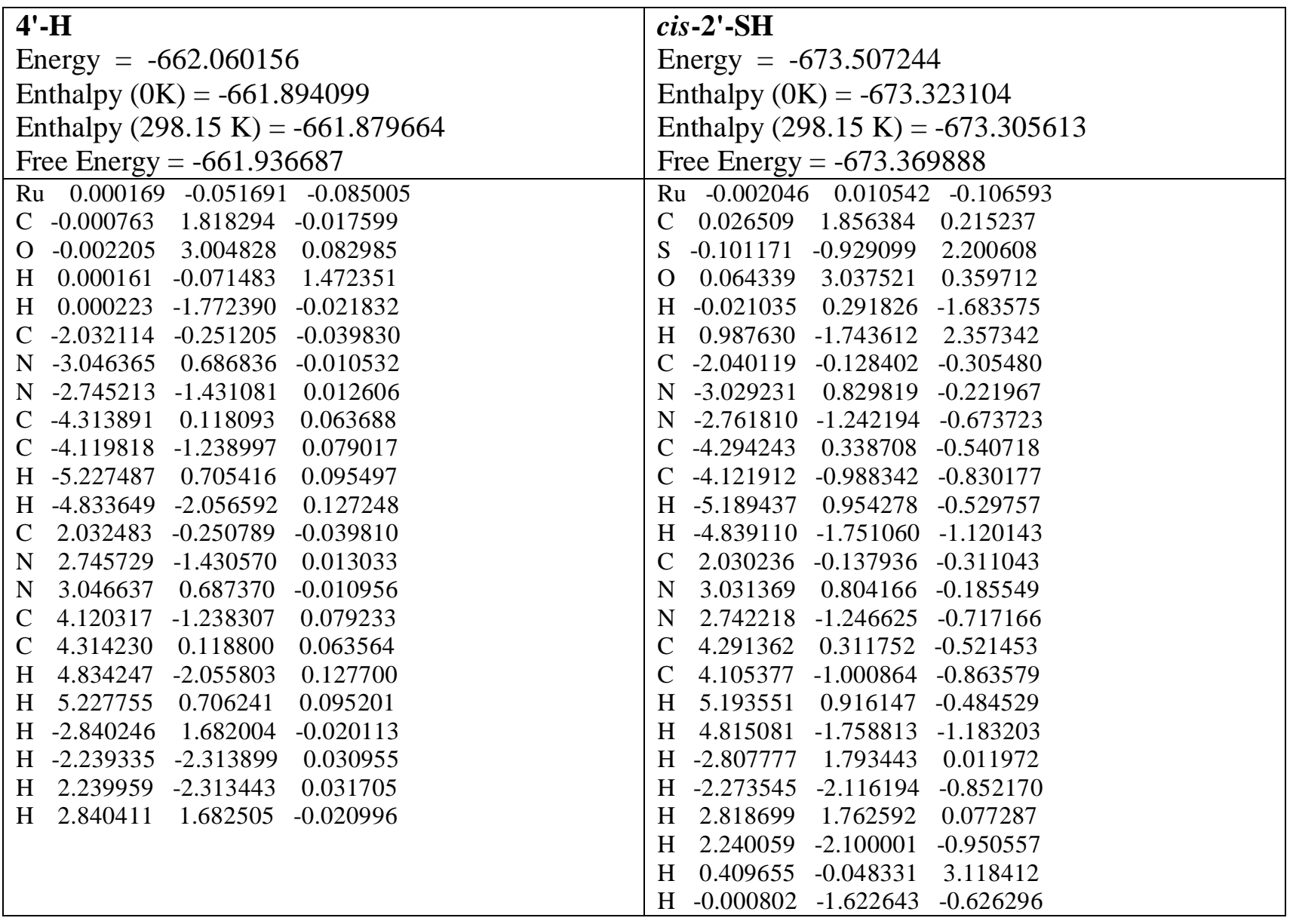




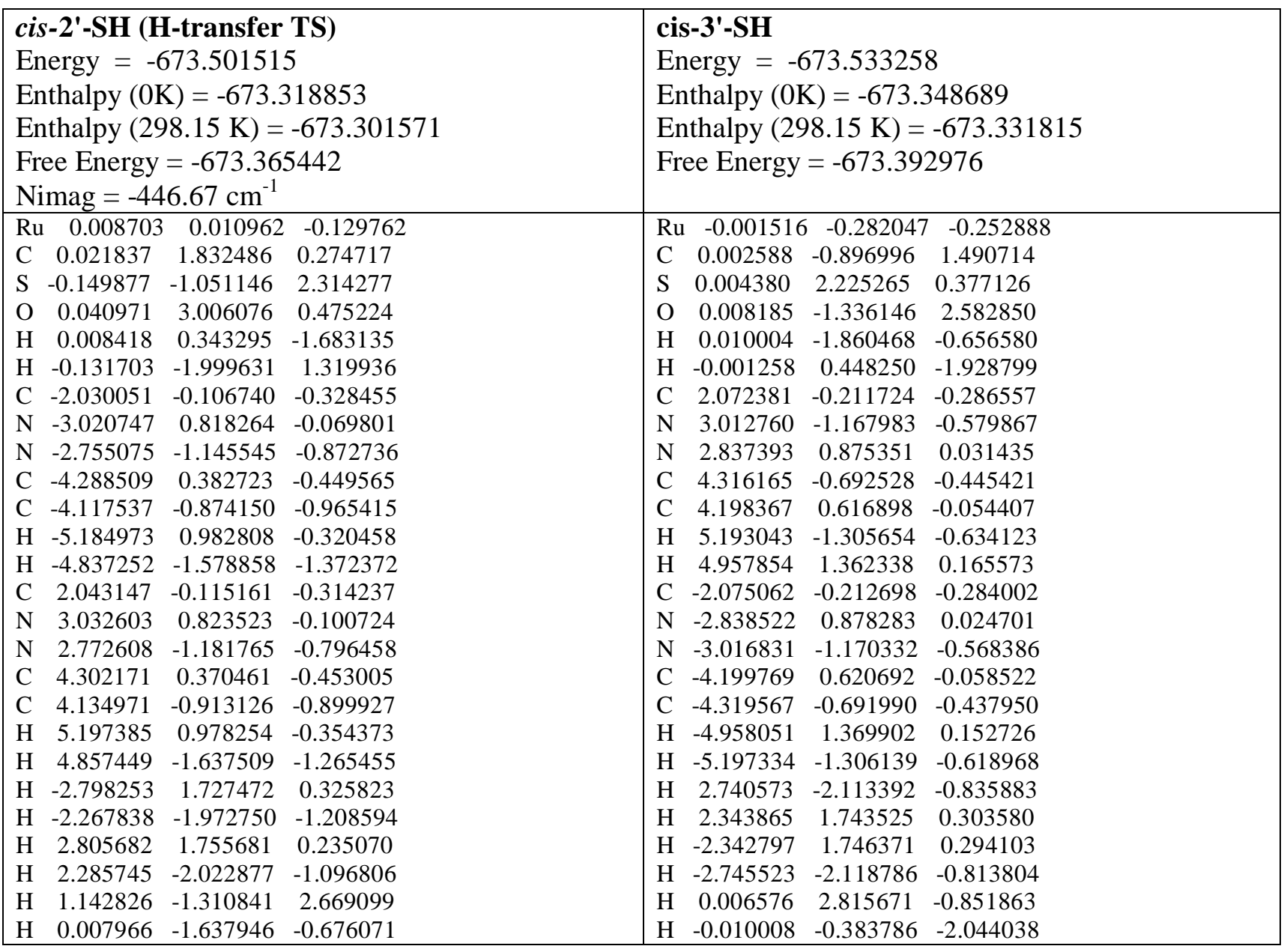




\begin{tabular}{|c|c|c|c|}
\hline \multicolumn{4}{|c|}{ 4'-SH } \\
\hline \multicolumn{4}{|c|}{ Energy $=-672.349526$} \\
\hline \multicolumn{4}{|c|}{ Enthalpy $(0 \mathrm{~K})=-672.181073$} \\
\hline \multicolumn{4}{|c|}{ Enthalpy $(298.15 \mathrm{~K})=-672.164841$} \\
\hline \multicolumn{4}{|c|}{ Free Energy $=-672.226023$} \\
\hline & 0.00000 & \begin{tabular}{|c|c|}
5 & 0.16771
\end{tabular} & -0.026996 \\
\hline & -0.000065 & 1.992898 & -0.056774 \\
\hline & -0.000160 & 3.184077 & -0.042000 \\
\hline & -0.000214 & 0.279733 & 1.523847 \\
\hline & -2.063832 & 0.054000 & -0.018163 \\
\hline & 2.063798 & 0.054064 & -0.017995 \\
\hline & -2.819886 & -1.087726 & -0.087610 \\
\hline & -4.182454 & -0.834640 & -0.052497 \\
\hline & -4.318310 & 0.527602 & 0.039373 \\
\hline & -3.024455 & 1.039498 & 0.056314 \\
\hline & 3.024387 & 1.039452 & 0.058379 \\
\hline & 4.318261 & 0.527645 & 0.040492 \\
\hline & 4.182475 & -0.834423 & -0.053991 \\
\hline & 2.819911 & -1.087500 & -0.089656 \\
\hline & -2.316115 & -1.990246 & -0.129023 \\
\hline & -2.776712 & 2.022989 & 0.127066 \\
\hline & 2.776599 & 2.022800 & 0.130935 \\
\hline $\mathrm{H}$ & 2.316219 & -1.989980 & -0.132569 \\
\hline & -4.931706 & -1.620352 & -0.097932 \\
\hline & -5.203813 & 1.155306 & 0.089598 \\
\hline & 5.203738 & 1.155289 & 0.091928 \\
\hline & 4.931763 & -1.620011 & -0.100942 \\
\hline & -0.000021 & -2.373709 & -0.010331 \\
\hline & 0.002707 & -2.536999 & 1.341566 \\
\hline
\end{tabular}




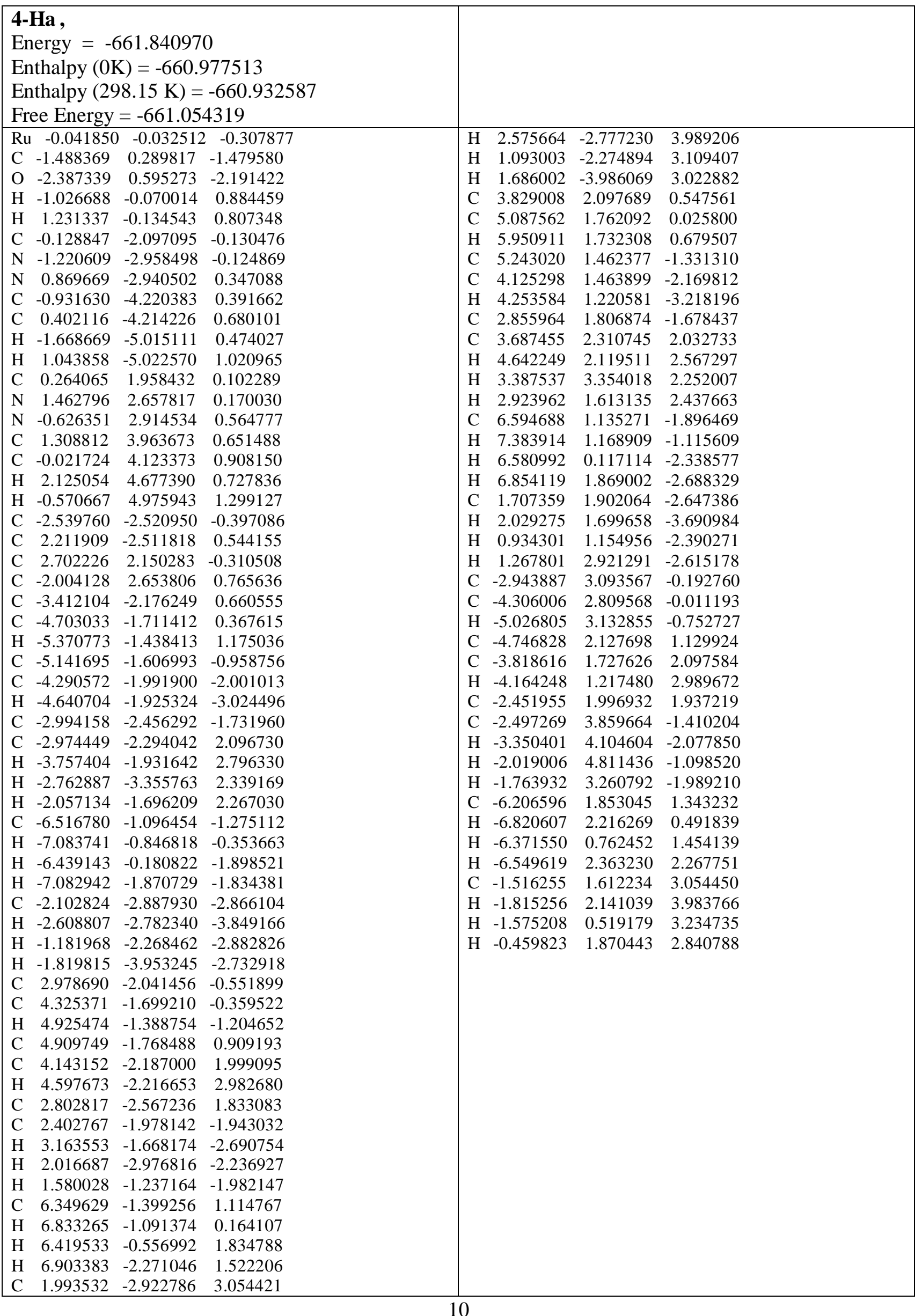




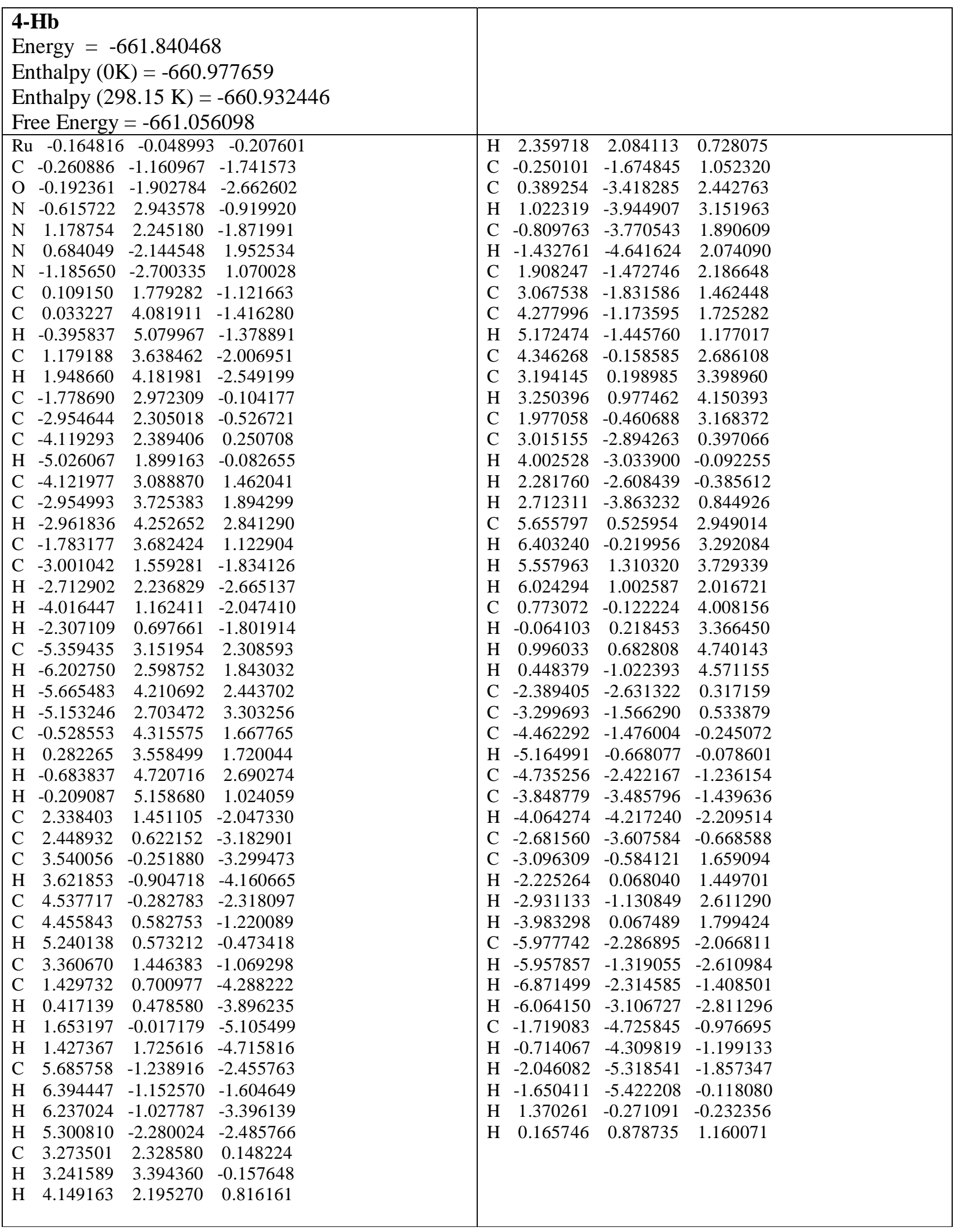




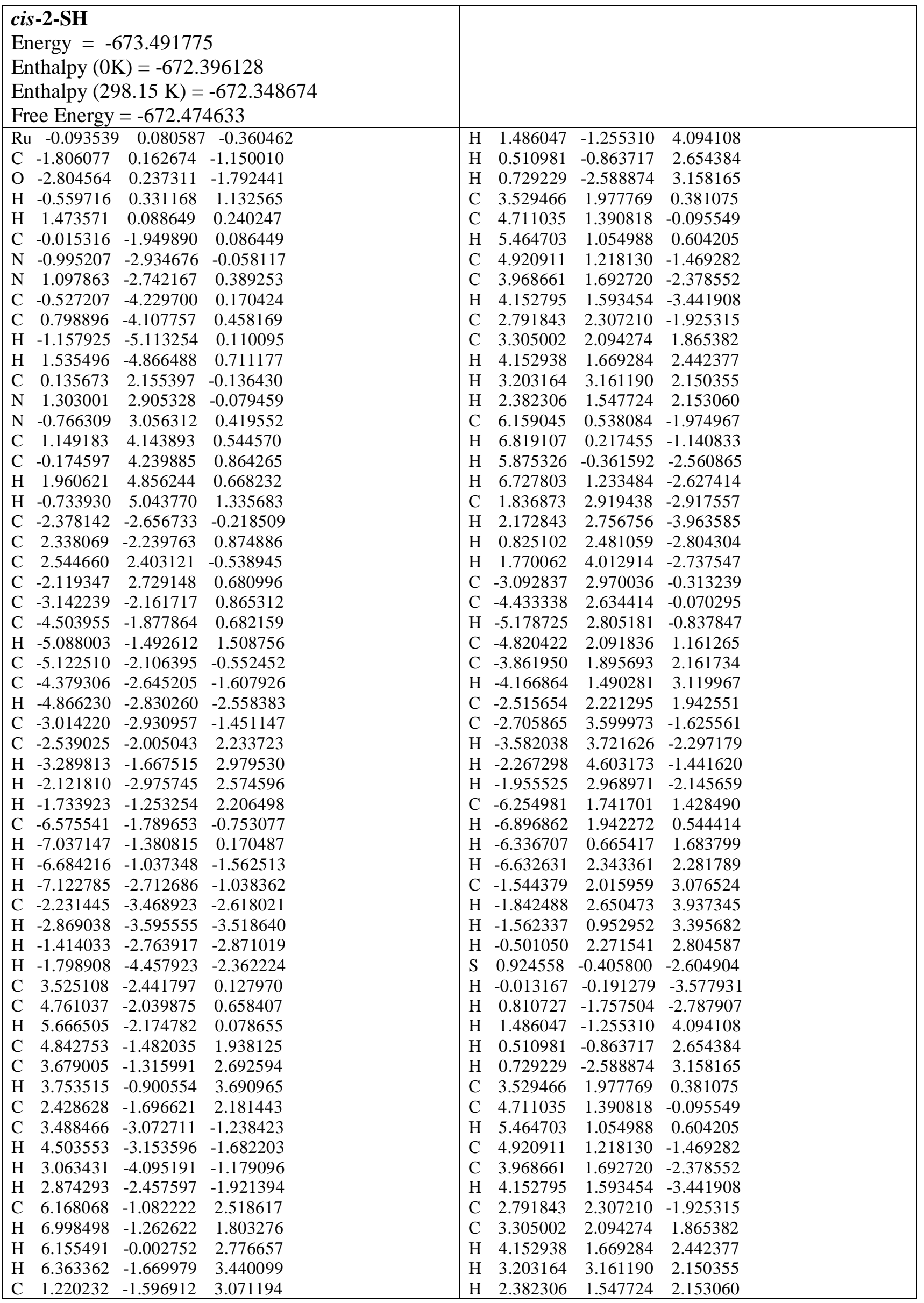




\begin{tabular}{|c|c|c|c|c|c|c|c|}
\hline \multicolumn{4}{|c|}{$\begin{array}{l}\text { cis-2-SH (H-transfer TS) } \\
\text { Energy = -673.272324 } \\
\text { Enthalpy }(0 \mathrm{~K})=-672.390582 \\
\text { Enthalpy }(298.15 \mathrm{~K})=-672.343414 \\
\text { Free Energy }=-672.468716 \\
\text { Nimag }=-434.29 \mathrm{~cm}^{-1}\end{array}$} & \multirow{2}{*}{\multicolumn{4}{|c|}{$\begin{array}{llll}\mathrm{H} & 6.571081 & -2.159172 & 2.986035\end{array}$}} \\
\hline & -0.09236 & 0.068328 & -0.269889 & & & & \\
\hline & -1.794905 & 0.167259 & -1.054821 & & 1.441110 & -1.840201 & 2.852085 \\
\hline & -2.796226 & 0.270922 & -1.686623 & $\mathrm{H}$ & 1.762489 & -1.556931 & 3.876824 \\
\hline & -0.613350 & 0.119272 & 1.208722 & $\mathrm{H}$ & 0.722447 & -1.077555 & 2.509588 \\
\hline $\mathrm{H}$ & 1.519378 & 0.029268 & 0.259929 & $\mathrm{H}$ & 0.937160 & -2.827843 & 2.909991 \\
\hline & 0.041307 & -2.010538 & -0.067969 & $\mathrm{C}$ & 3.159071 & 2.017670 & 0.752324 \\
\hline & -0.941142 & -2.988655 & -0.231924 & $\mathrm{C}$ & 4.462918 & 1.548155 & 0.540706 \\
\hline $\mathrm{N}$ & 1.170414 & -2.824896 & 0.113706 & $\mathrm{H}$ & 5.023644 & 1.137204 & 1.368297 \\
\hline & -0.464518 & -4.295580 & -0.139093 & $\mathrm{C}$ & 5.052498 & 1.617967 & -0.727224 \\
\hline & 0.874126 & -4.192810 & 0.086360 & $\mathrm{C}$ & 4.354130 & 2.217287 & -1.781170 \\
\hline $\mathrm{H}$ & -1.100833 & -5.171990 & -0.234073 & $\mathrm{H}$ & 4.828039 & 2.299947 & -2.752325 \\
\hline $\mathrm{H}$ & 1.620166 & -4.968447 & 0.241906 & $\mathrm{C}$ & 3.059408 & 2.721629 & -1.586466 \\
\hline $\mathrm{C}$ & 0.020229 & $2.139256-$ & -0.009093 & C & 2.572689 & 1.978434 & 2.140112 \\
\hline $\mathrm{N}$ & 1.099637 & 3.006531 & -0.146085 & $\mathrm{H}$ & 3.287768 & 1.553770 & 2.876349 \\
\hline $\mathrm{N}$ & -0.987923 & 3.047230 & 0.311748 & $\mathrm{H}$ & 2.312430 & 3.007831 & 2.464111 \\
\hline $\mathrm{C}$ & 0.778484 & 4.348229 & 0.069614 & $\mathrm{H}$ & 1.658926 & 1.352787 & 2.158400 \\
\hline & -0.553929 & 4.373132 & 0.358716 & C & 6.431918 & 1.076704 & -0.962757 \\
\hline $\mathrm{H}$ & 1.505784 & 5.153688 & 0.010215 & $\mathrm{H}$ & 6.865901 & 0.644873 & -0.036475 \\
\hline $\mathrm{H}$ & -1.207567 & 5.206332 & 0.603864 & $\mathrm{H}$ & 6.391423 & 0.279546 & -1.734697 \\
\hline C & -2.327195 & -2.708074 & -0.334159 & $\mathrm{H}$ & 7.098211 & 1.891846 & -1.315352 \\
\hline C & 2.439754 & -2.370399 & 0.569150 & C & 2.351908 & 3.415190 & -2.720996 \\
\hline C & 2.432189 & 2.563365 & -0.330505 & $\mathrm{H}$ & 2.961992 & 3.411518 & -3.649111 \\
\hline C & -2.308559 & 2.668101 & 0.668460 & $\mathrm{H}$ & 1.387452 & 2.912292 & -2.937081 \\
\hline $\mathrm{C}$ & -3.072431 & -2.348301 & 0.813111 & $\mathrm{H}$ & 2.151670 & 4.471477 & -2.446670 \\
\hline C & -4.439631 & -2.058191 & 0.687574 & C & -3.377933 & 2.934124 & -0.219101 \\
\hline $\mathrm{H}$ & -5.011532 & -1.777324 & 1.563258 & C & -4.674092 & 2.504652 & 0.105691 \\
\hline C & -5.079447 & -2.151597 & -0.554686 & $\mathrm{H}$ & -5.489951 & 2.685378 & -0.584012 \\
\hline C & -4.351734 & -2.559341 & -1.678087 & C & -4.925986 & 1.843606 & 1.313627 \\
\hline $\mathrm{H}$ & -4.854772 & -2.640899 & -2.634594 & $\mathrm{C}$ & -3.877854 & 1.627015 & 2.213631 \\
\hline C & -2.982215 & -2.848372 & -1.578562 & $\mathrm{H}$ & -4.082625 & 1.147009 & 3.164066 \\
\hline C & -2.441242 & -2.347028 & 2.179517 & C & -2.575732 & 2.054477 & 1.918083 \\
\hline $\mathrm{H}$ & -3.175157 & -2.088620 & 2.972191 & C & -3.135366 & 3.624566 & -1.535898 \\
\hline $\mathrm{H}$ & -2.030483 & -3.354068 & 2.402390 & $\mathrm{H}$ & -4.065982 & 3.702183 & -2.137206 \\
\hline $\mathrm{H}$ & -1.623725 & -1.607158 & 2.224629 & $\mathrm{H}$ & -2.757134 & 4.652323 & -1.360029 \\
\hline C & -6.539051 & -1.832031 & -0.693133 & $\mathrm{H}$ & -2.387489 & 3.056900 & -2.128415 \\
\hline $\mathrm{H}$ & -6.989624 & -1.542014 & 0.279267 & C & -6.313405 & 1.395898 & 1.669041 \\
\hline $\mathrm{H}$ & -6.669610 & -0.990430 & -1.405628 & $\mathrm{H}$ & -7.037998 & 1.624078 & 0.858663 \\
\hline $\mathrm{H}$ & -7.081092 & -2.721613 & -1.077422 & $\mathrm{H}$ & -6.319691 & 0.301454 & 1.847245 \\
\hline $\mathrm{C}$ & -2.215043 & -3.249643 & -2.809767 & $\mathrm{H}$ & -6.645433 & 1.912155 & 2.594219 \\
\hline $\mathrm{H}$ & -2.865728 & -3.275433 & -3.709528 & C & -1.533014 & 1.927495 & 2.998598 \\
\hline $\mathrm{H}$ & -1.397591 & -2.521594 & -2.994238 & $\mathrm{H}$ & -1.862907 & 2.493902 & 3.894799 \\
\hline $\mathrm{H}$ & -1.780034 & -4.260937 & -2.671281 & $\mathrm{H}$ & -1.410035 & 0.861803 & 3.284019 \\
\hline C & 3.584695 & -2.553532 & -0.245050 & $\mathrm{H}$ & -0.547984 & 2.330932 & 2.693879 \\
\hline C & 4.855363 & -2.224832 & 0.252087 & $\mathrm{~S}$ & 1.018343 & 0.034729 & -2.707666 \\
\hline $\mathrm{H}$ & 5.729496 & -2.355104 & -0.374826 & $\mathrm{H}$ & 0.886428 & -1.273510 & -3.068330 \\
\hline C & 5.013384 & -1.767161 & 1.564219 & $\mathrm{H}$ & 1.924403 & -0.235195 & -1.713796 \\
\hline C & 3.888194 & -1.602464 & 2.376695 & & & & \\
\hline $\mathrm{H}$ & 4.020556 & -1.259452 & 3.396285 & & & & \\
\hline C & 2.604158 & -1.911341 & 1.900592 & & & & \\
\hline C & 3.465145 & -3.097985 & -1.644536 & & & & \\
\hline $\mathrm{H}$ & 4.449913 & -3.136566 & -2.156858 & & & & \\
\hline $\mathrm{H}$ & 3.057066 & -4.128548 & -1.620788 & & & & \\
\hline $\mathrm{H}$ & 2.793931 & -2.455575 & -2.245481 & & & & \\
\hline C & 6.379983 & -1.489393 & 2.121403 & & & & \\
\hline $\mathrm{H}$ & 7.173557 & -1.660829 & 1.363364 & & & & \\
\hline & 6.444142 & -0.436267 & 2.464420 & & & & \\
\hline
\end{tabular}




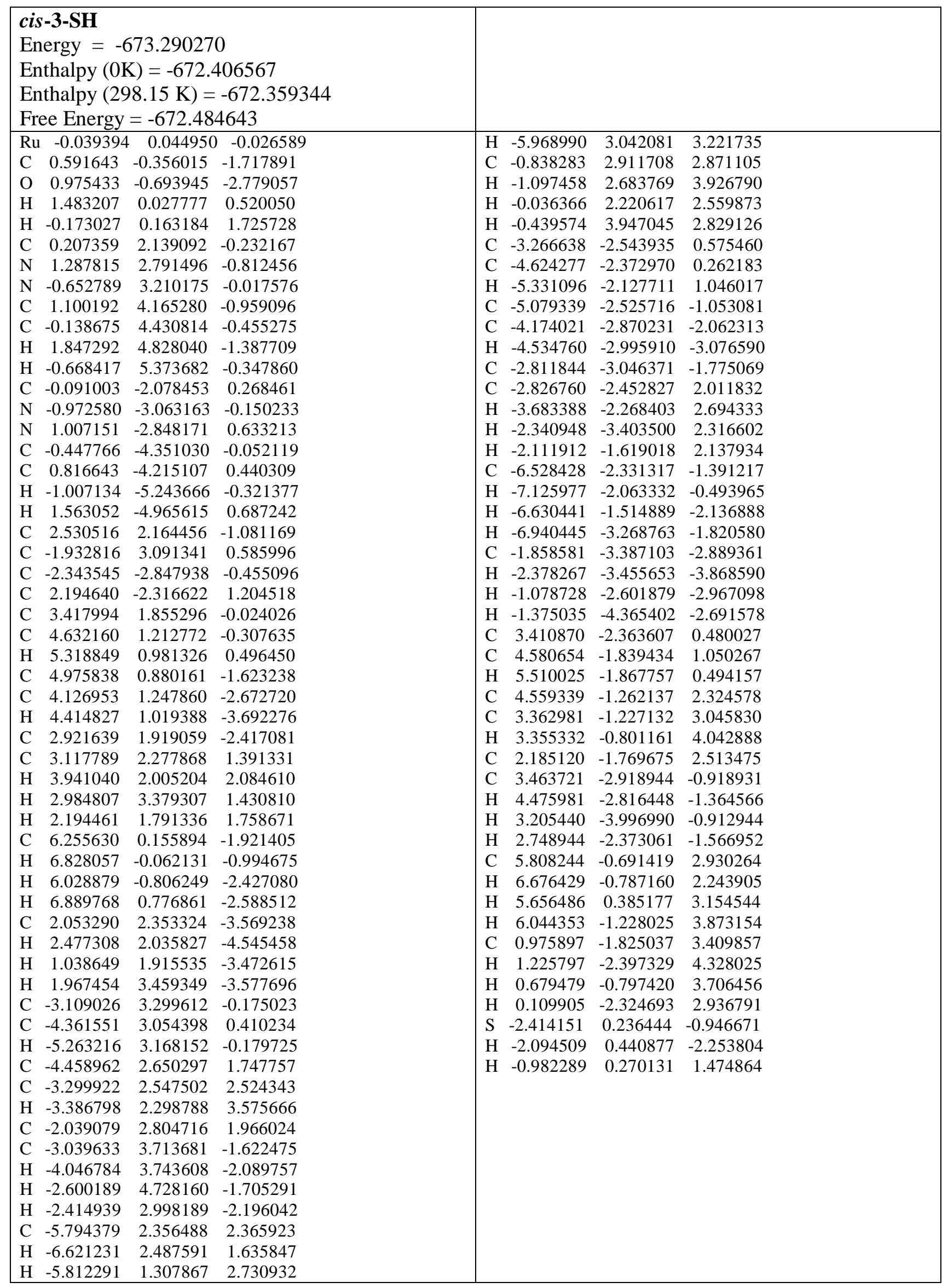




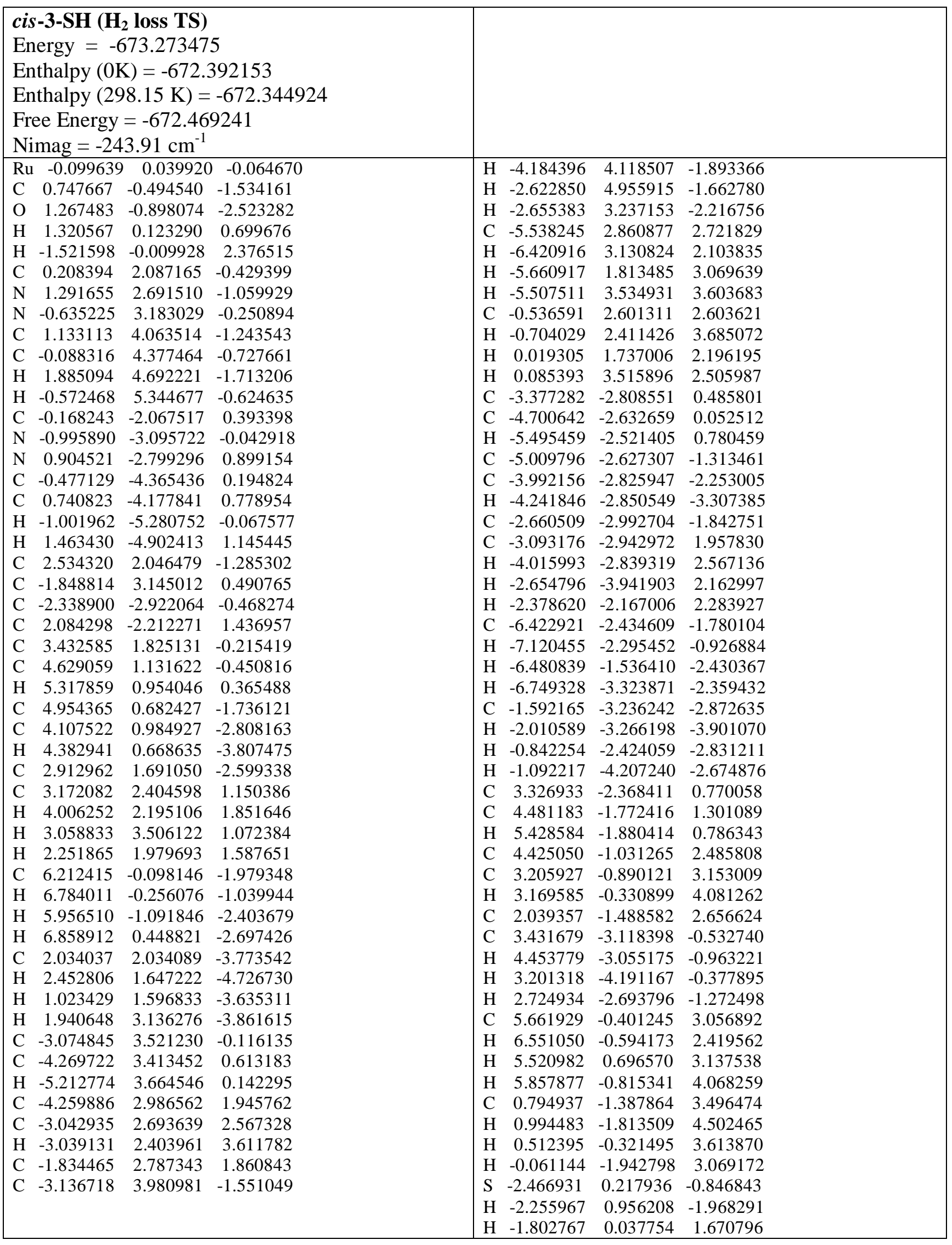




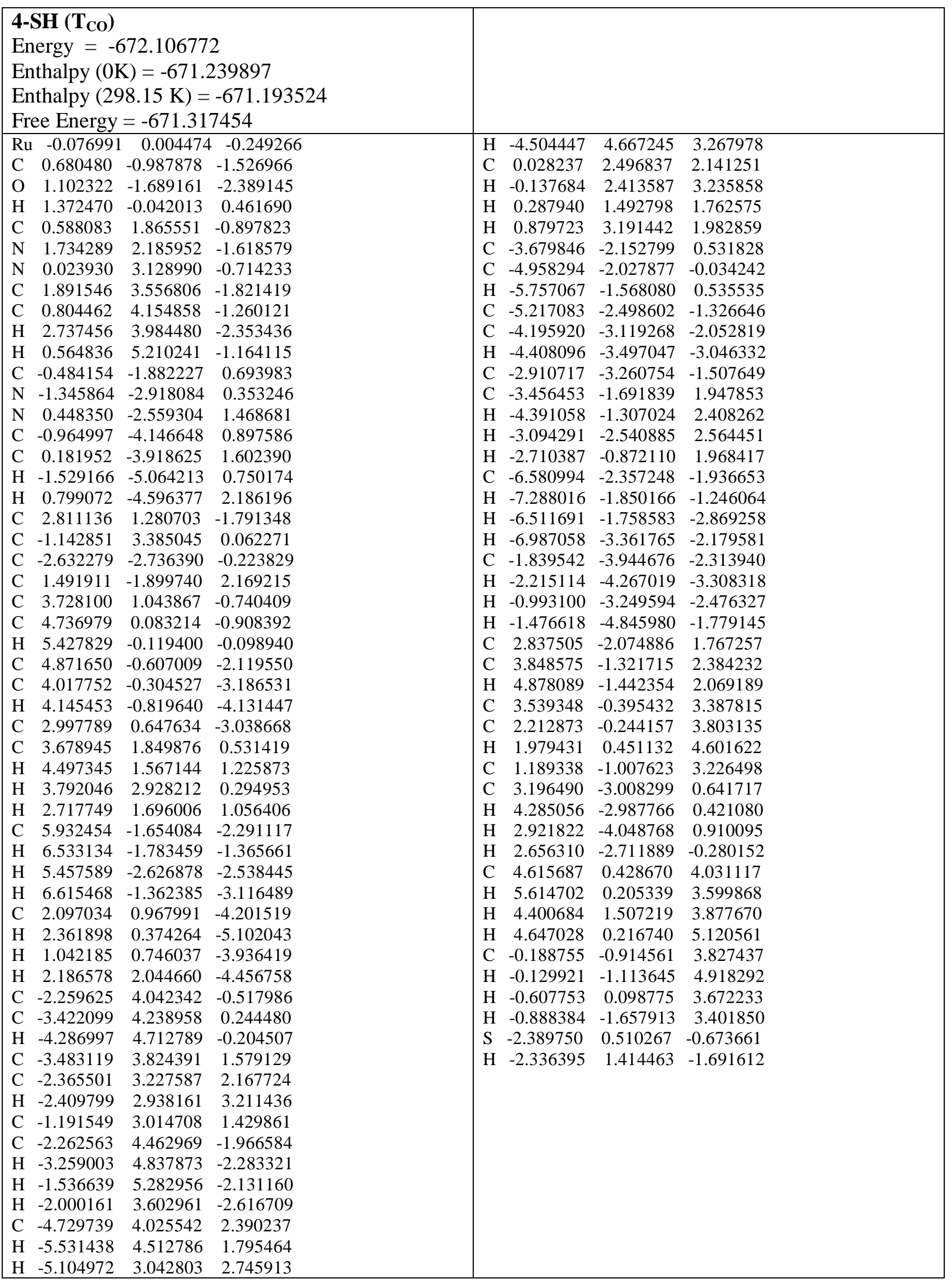




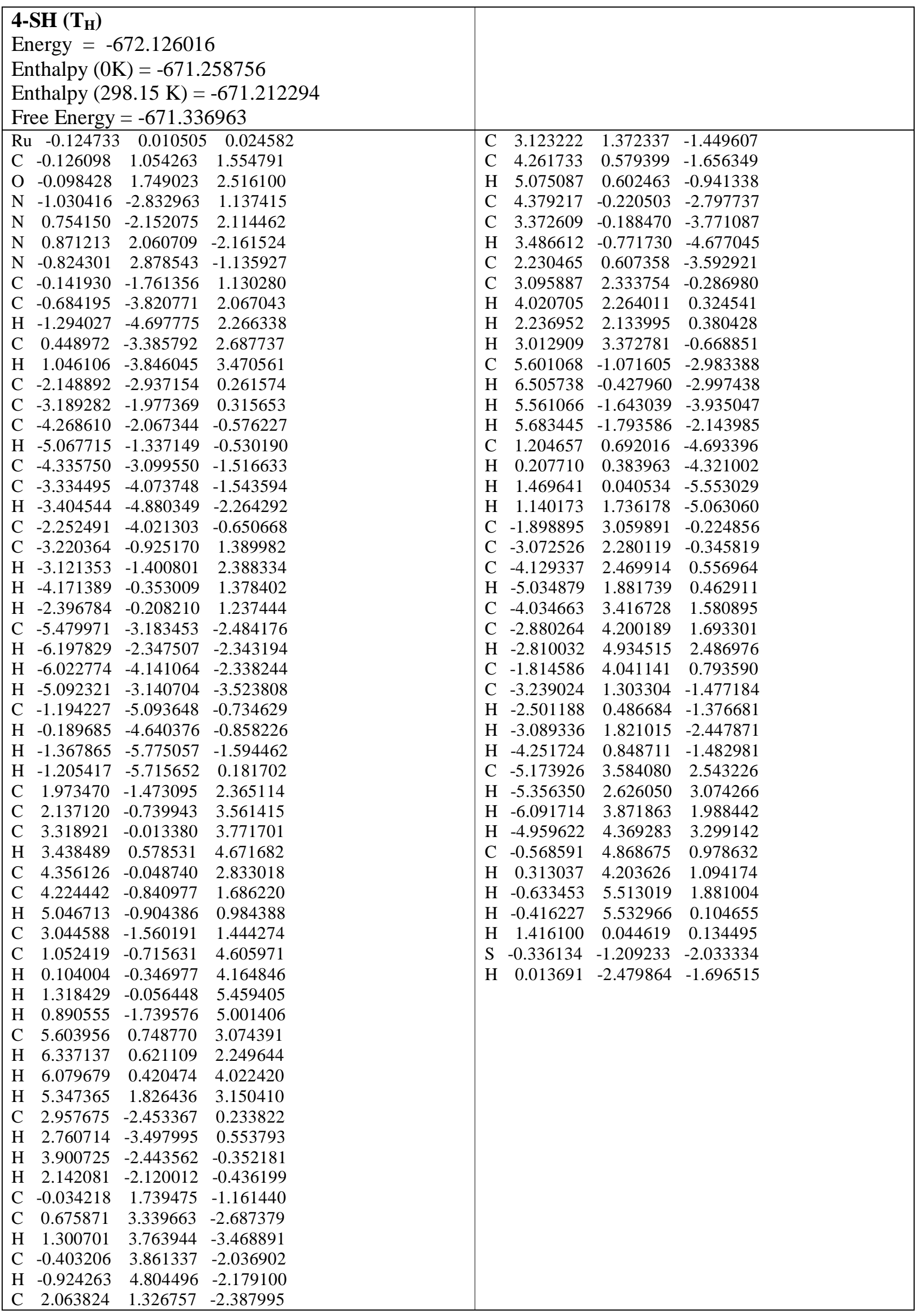




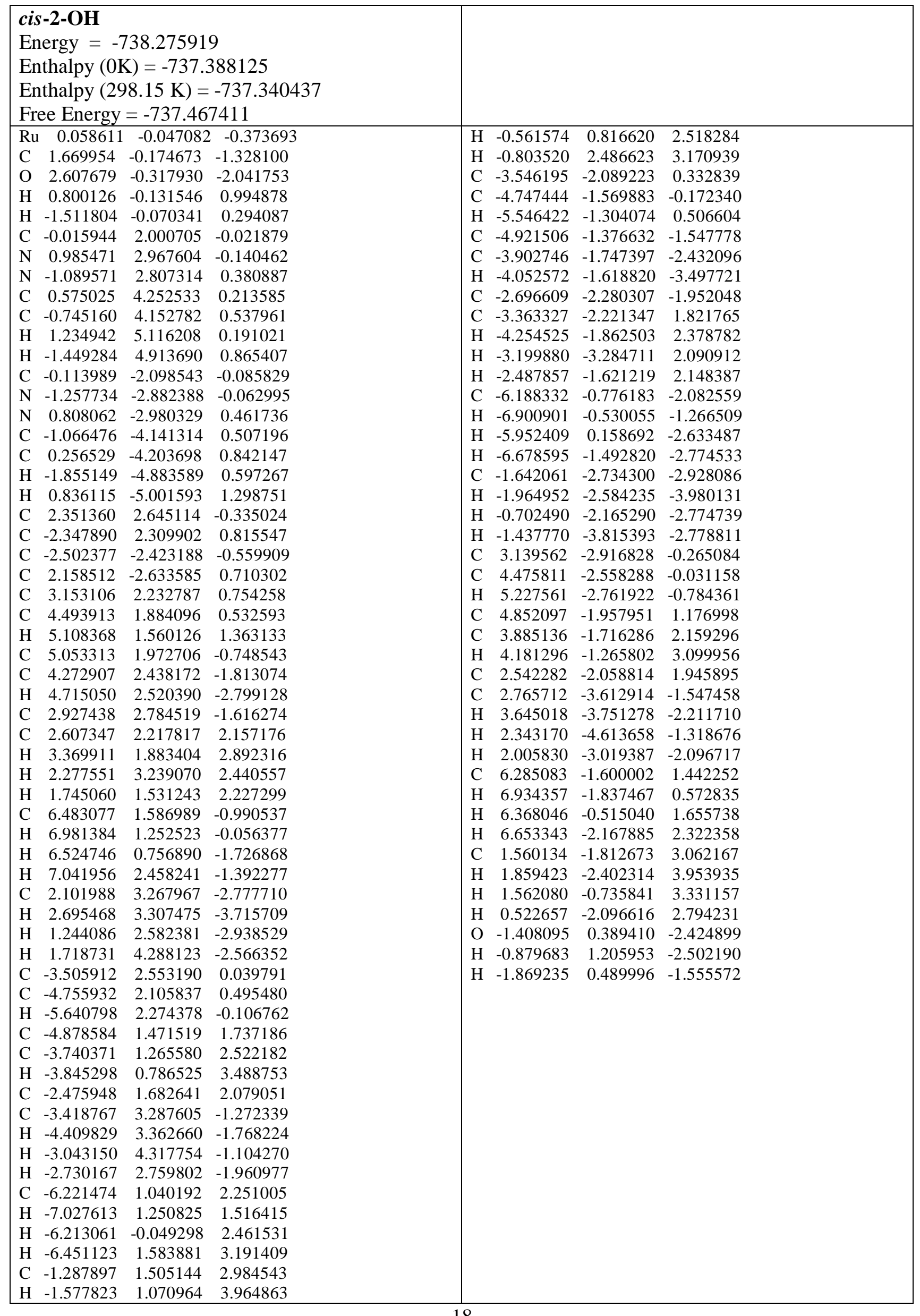




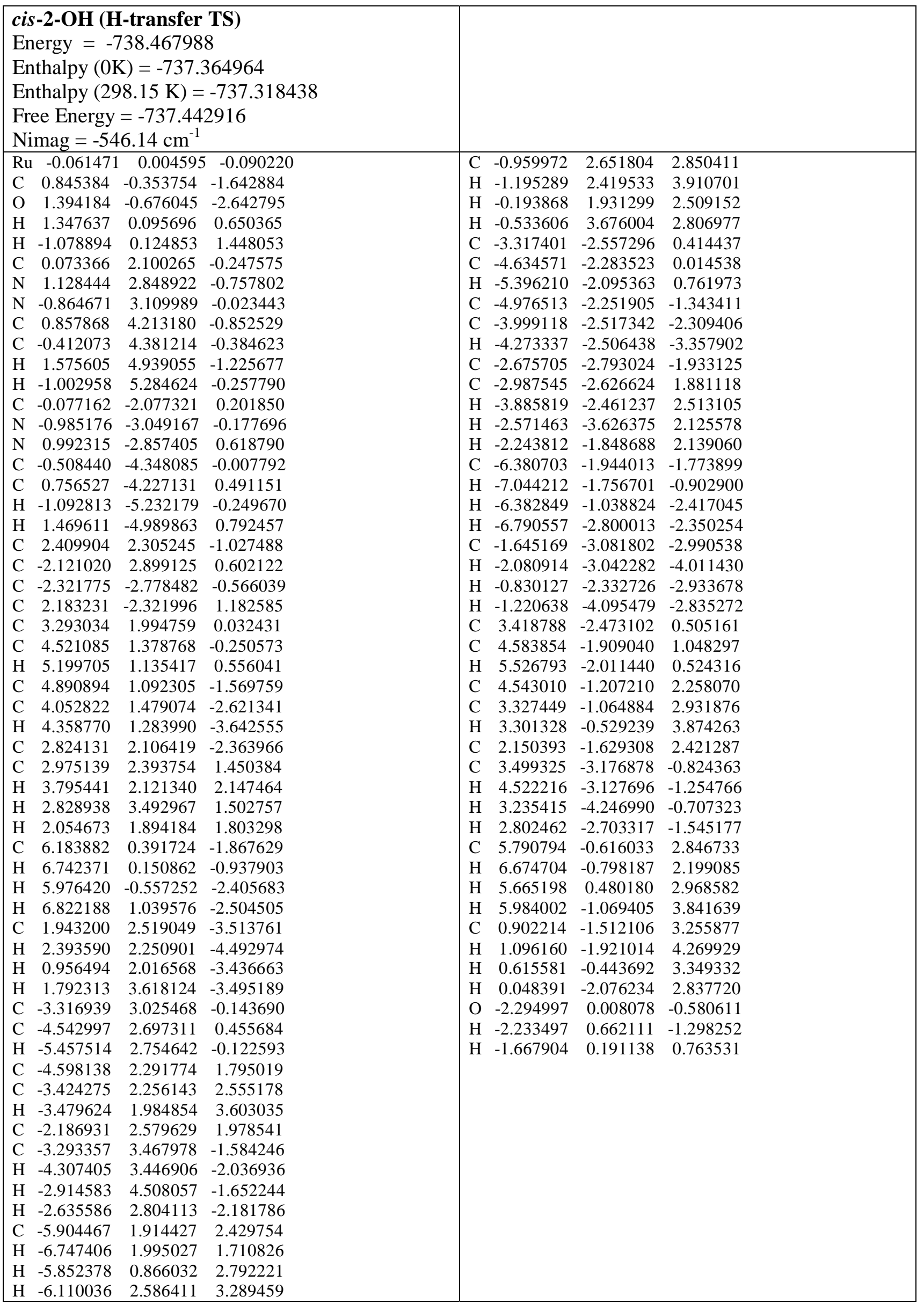




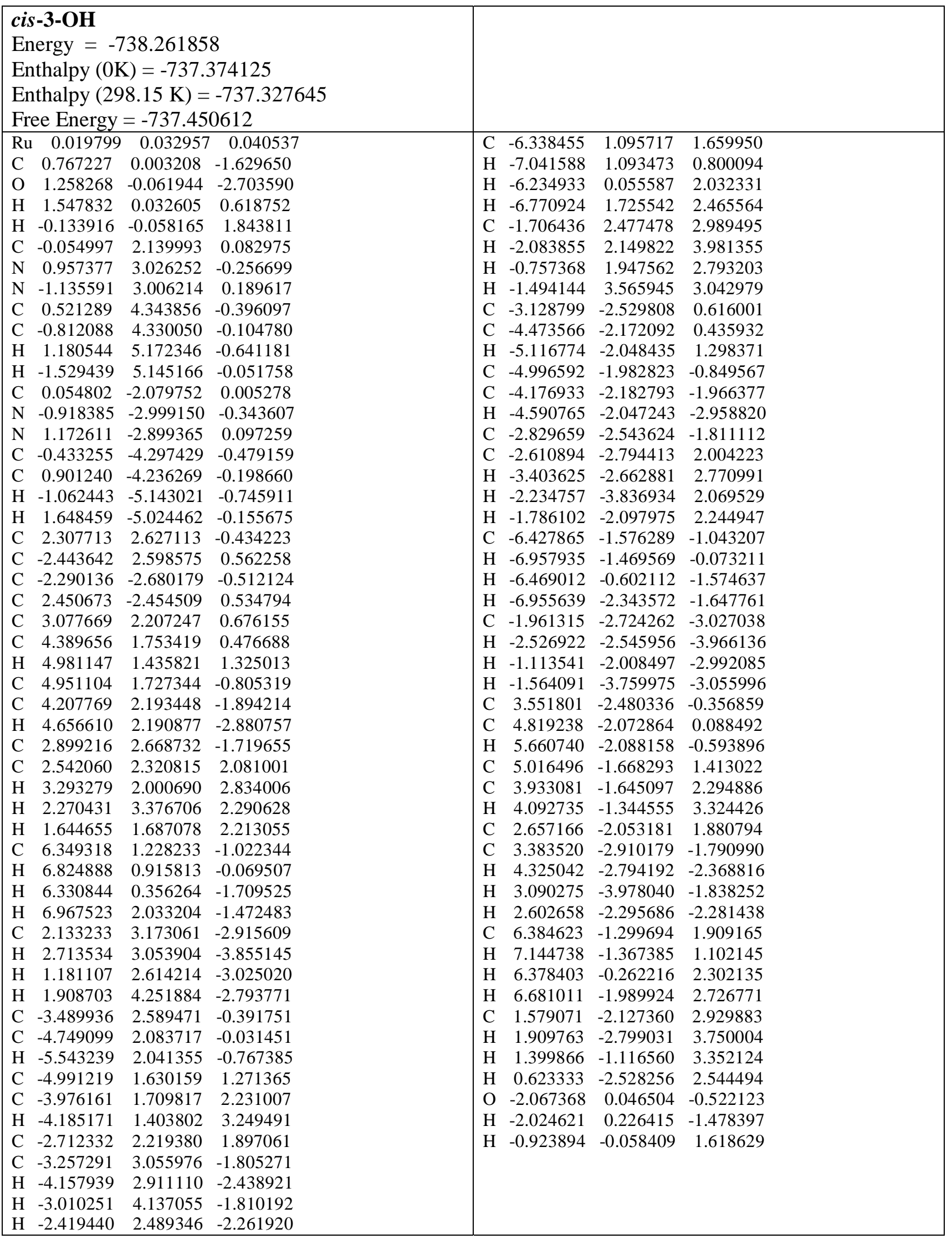




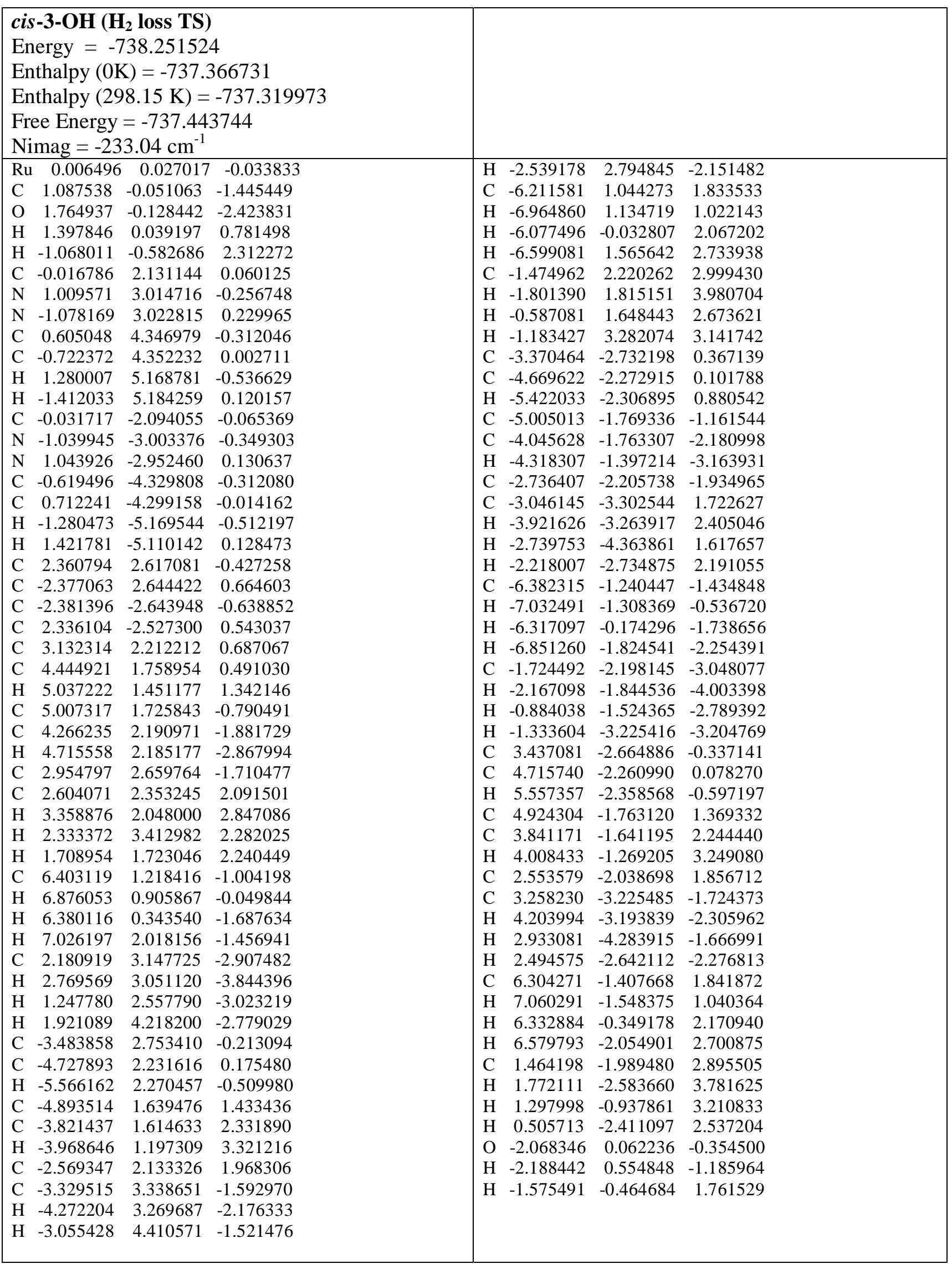




\begin{tabular}{|c|c|c|c|c|c|c|c|}
\hline \multicolumn{4}{|c|}{$\begin{array}{l}\text { 4-OH }\left(\mathbf{T}_{\mathbf{C o}}\right) \\
\text { Energy }=-737.088779 \\
\text { Enthalpy }(0 \mathrm{~K})=-736.218291 \\
\text { Enthalpy }(298.15 \mathrm{~K})=-736.172348 \\
\text { Free Energy }=-736.29529\end{array}$} & \multirow{2}{*}{\multicolumn{4}{|c|}{$\begin{array}{llll}\mathrm{H} & 6.243985 & 1.659911 & -3.140177\end{array}$}} \\
\hline & -0.029432 & $2 \quad 0.052327$ & 0.332474 & & & & \\
\hline & -1.446534 & -0.269366 & 1.393157 & $\mathrm{C}$ & 1.115575 & 1.158060 & -2.725235 \\
\hline & -2.304618 & -0.500450 & 2.188017 & $\mathrm{H}$ & 1.441815 & 0.555650 & -3.599556 \\
\hline & -1.202750 & 0.019904 & -0.756741 & $\mathrm{H}$ & 0.503643 & 0.501625 & -2.085306 \\
\hline & -0.165171 & 2.099435 & 0.089447 & $\mathrm{H}$ & 0.499535 & 2.003242 & -3.097818 \\
\hline & -1.233521 & 2.972646 & 0.272744 & $\mathrm{C}$ & 3.834970 & -2.150861 & -0.190400 \\
\hline $\mathrm{N}$ & 0.797137 & 2.966803 & -0.441790 & $\mathrm{C}$ & 5.014650 & -1.745522 & 0.452068 \\
\hline & -0.970275 & 4.270379 & -0.167732 & $\mathrm{H}$ & 5.905249 & -1.547715 & -0.132234 \\
\hline & 0.321238 & 4.273216 & -0.599216 & $\mathrm{C}$ & 5.053268 & -1.584792 & 1.841960 \\
\hline & -1.699275 & 5.074876 & -0.113169 & $\mathrm{C}$ & 3.905527 & -1.839253 & 2.599866 \\
\hline $\mathrm{H}$ & 0.911951 & 5.076158 & -1.032814 & $\mathrm{H}$ & 3.946589 & -1.722806 & 3.676667 \\
\hline $\mathrm{C}$ & 0.291051 & -1.999096 & -0.061423 & $\mathrm{C}$ & 2.709412 & -2.235674 & 1.981998 \\
\hline & 1.456251 & -2.754548 & -0.074744 & $\mathrm{C}$ & 3.813607 & -2.254018 & -1.693192 \\
\hline $\mathrm{N}$ & -0.582902 & -2.855651 & -0.713600 & $\mathrm{H}$ & 4.779294 & -1.938314 & -2.141600 \\
\hline & 1.300213 & -3.992237 & -0.700530 & $\mathrm{H}$ & 3.626611 & -3.301778 & -2.002260 \\
\hline $\mathrm{C}$ & -0.004345 & -4.061750 & -1.096951 & $\mathrm{H}$ & 3.013073 & -1.602928 & -2.102599 \\
\hline $\mathrm{H}$ & 2.096675 & -4.727890 & -0.780742 & $\mathrm{C}$ & 6.312008 & -1.140846 & 2.527926 \\
\hline $\mathrm{H}$ & -0.548393 & -4.847528 & -1.614358 & $\mathrm{H}$ & 7.137272 & -0.975853 & 1.802859 \\
\hline & -2.552913 & 2.517060 & 0.512855 & $\mathrm{H}$ & 6.125466 & -0.189203 & 3.068847 \\
\hline $\mathrm{C}$ & 2.103517 & 2.584320 & -0.861134 & $\mathrm{H}$ & 6.633382 & -1.915343 & 3.255774 \\
\hline C & 2.664859 & -2.380732 & 0.573283 & $\mathrm{C}$ & 1.504010 & -2.533695 & 2.834129 \\
\hline $\mathrm{C}$ & -1.965686 & -2.587853 & -0.867596 & $\mathrm{H}$ & 1.733789 & -2.443738 & 3.917053 \\
\hline $\mathrm{C}$ & -3.385914 & 2.131966 & -0.563168 & & 0.689234 & -1.818858 & 2.604131 \\
\hline $\mathrm{C}$ & -4.675138 & 1.646393 & -0.298157 & $\mathrm{H}$ & 1.153354 & -3.569294 & 2.640253 \\
\hline $\mathrm{H}$ & -5.313776 & 1.345800 & -1.118898 & $\mathrm{C}$ & -2.875198 & -2.999607 & 0.132463 \\
\hline & -5.152326 & 1.564043 & 1.016456 & $\mathrm{C}$ & -4.244449 & -2.734609 & -0.023039 \\
\hline $\mathrm{C}$ & -4.342878 & 1.994092 & 2.074267 & $\mathrm{H}$ & -4.942671 & -3.039927 & 0.747155 \\
\hline $\mathrm{H}$ & -4.722412 & 1.943293 & 3.088127 & $\mathrm{C}$ & -4.722938 & -2.106578 & -1.179898 \\
\hline $\mathrm{C}$ & -3.048456 & 2.478838 & 1.833270 & $\mathrm{C}$ & -3.824227 & -1.731910 & -2.184919 \\
\hline $\mathrm{C}$ & -2.922180 & 2.262697 & -1.990654 & $\mathrm{H}$ & -4.197616 & -1.255224 & -3.084251 \\
\hline $\mathrm{H}$ & -3.686985 & 1.893945 & -2.706788 & $\mathrm{C}$ & -2.450407 & -1.975767 & -2.047574 \\
\hline $\mathrm{H}$ & -2.720162 & 3.328975 & -2.221969 & $\mathrm{C}$ & -2.393689 & -3.744158 & 1.350550 \\
\hline $\mathrm{H}$ & -1.994764 & 1.680009 & -2.151653 & $\mathrm{H}$ & -3.228504 & -3.983089 & 2.043012 \\
\hline $\mathrm{C}$ & -6.525371 & 1.031053 & 1.303161 & $\mathrm{H}$ & -1.918145 & -4.698167 & 1.041191 \\
\hline $\mathrm{H}$ & -7.057185 & 0.745080 & 0.371435 & $\mathrm{H}$ & -1.649405 & -3.135573 & 1.903383 \\
\hline $\mathrm{H}$ & -6.447770 & 0.133624 & 1.952477 & $\mathrm{C}$ & -6.191794 & -1.864331 & -1.369537 \\
\hline $\mathrm{H}$ & -7.125601 & 1.806441 & 1.824036 & $\mathrm{H}$ & -6.779393 & -2.199278 & -0.488415 \\
\hline $\mathrm{C}$ & -2.192543 & 2.935971 & 2.983506 & $\mathrm{H}$ & -6.378400 & -0.782660 & -1.524934 \\
\hline $\mathrm{H}$ & -2.722118 & 2.836594 & 3.954745 & $\mathrm{H}$ & -6.547656 & -2.420449 & -2.262157 \\
\hline $\mathrm{H}$ & -1.266043 & 2.325807 & 3.028722 & $\mathrm{C}$ & -1.538986 & -1.575784 & -3.178244 \\
\hline $\mathrm{H}$ & -1.918313 & 4.002732 & 2.843817 & $\mathrm{H}$ & -1.831162 & -2.122058 & -4.099496 \\
\hline $\mathrm{C}$ & 3.241750 & 3.124189 & -0.209533 & $\mathrm{H}$ & -1.632796 & -0.485850 & -3.364904 \\
\hline $\mathrm{C}$ & 4.526838 & 2.723514 & -0.607063 & $\mathrm{H}$ & -0.472851 & -1.797819 & -2.970658 \\
\hline $\mathrm{H}$ & 5.396411 & 3.114245 & -0.092002 & $\mathrm{O}$ & 1.956396 & 0.246856 & 0.730519 \\
\hline $\mathrm{C}$ & 4.700850 & 1.816378 & -1.657343 & $\mathrm{H}$ & 2.073820 & 1.035159 & 1.291032 \\
\hline $\mathrm{C}$ & 3.582195 & 1.312067 & -2.325361 & & & & \\
\hline $\mathrm{H}$ & 3.727462 & 0.627251 & -3.152760 & & & & \\
\hline $\mathrm{C}$ & 2.284657 & 1.681568 & -1.938691 & & & & \\
\hline C & 3.098674 & 4.054354 & 0.967871 & & & & \\
\hline $\mathrm{H}$ & 4.082983 & 4.299937 & 1.420209 & & & & \\
\hline $\mathrm{H}$ & 2.631449 & 5.007745 & 0.650585 & & & & \\
\hline $\mathrm{H}$ & 2.471495 & 3.577925 & 1.750501 & & & & \\
\hline $\mathrm{C}$ & 6.074480 & 1.382775 & -2.078458 & & & & \\
\hline $\mathrm{H}$ & 6.862932 & 1.861653 & -1.459756 & & & & \\
\hline $\mathrm{H}$ & 6.167112 & 0.281823 & -1.972486 & & & & \\
\hline
\end{tabular}




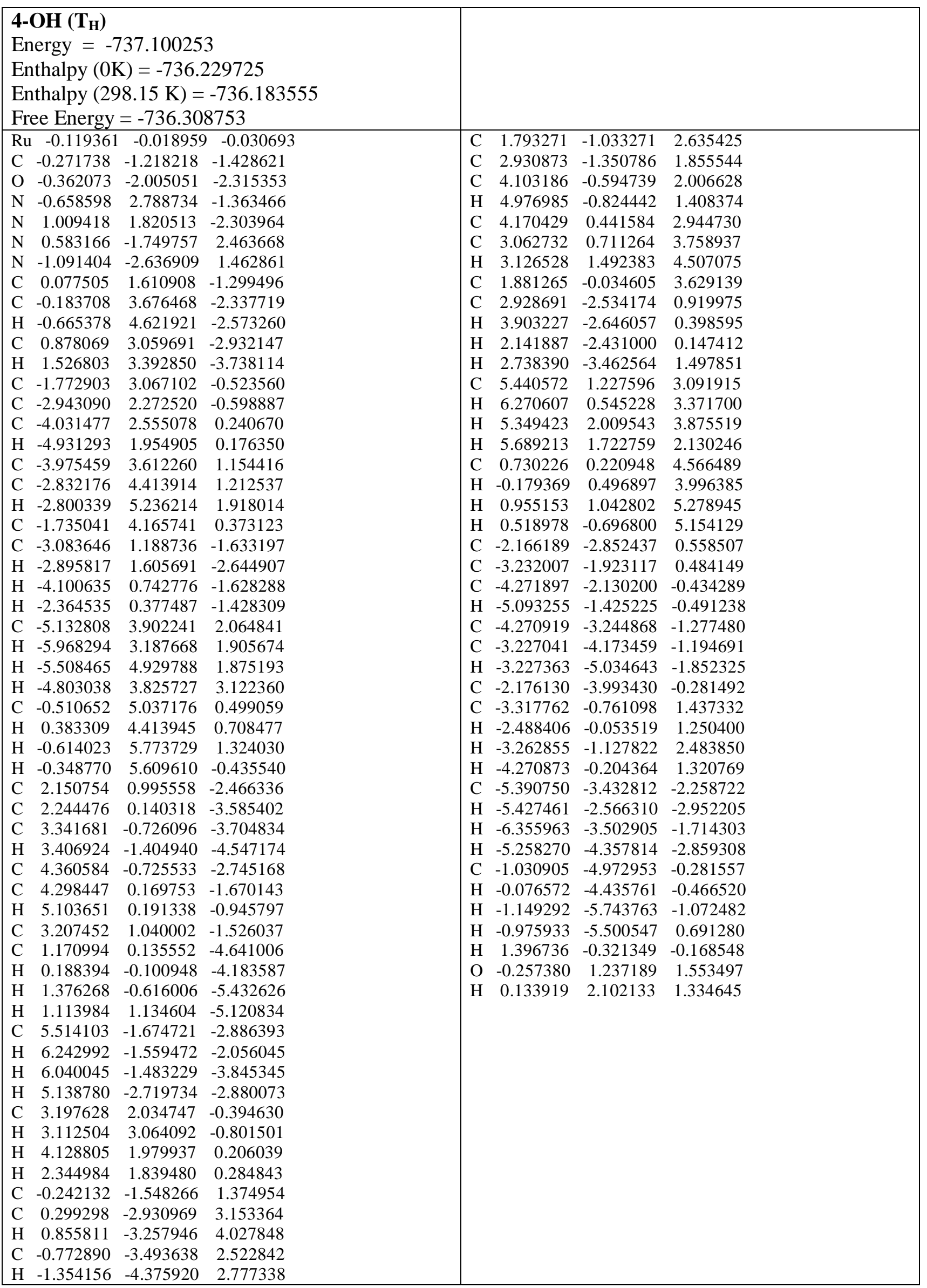




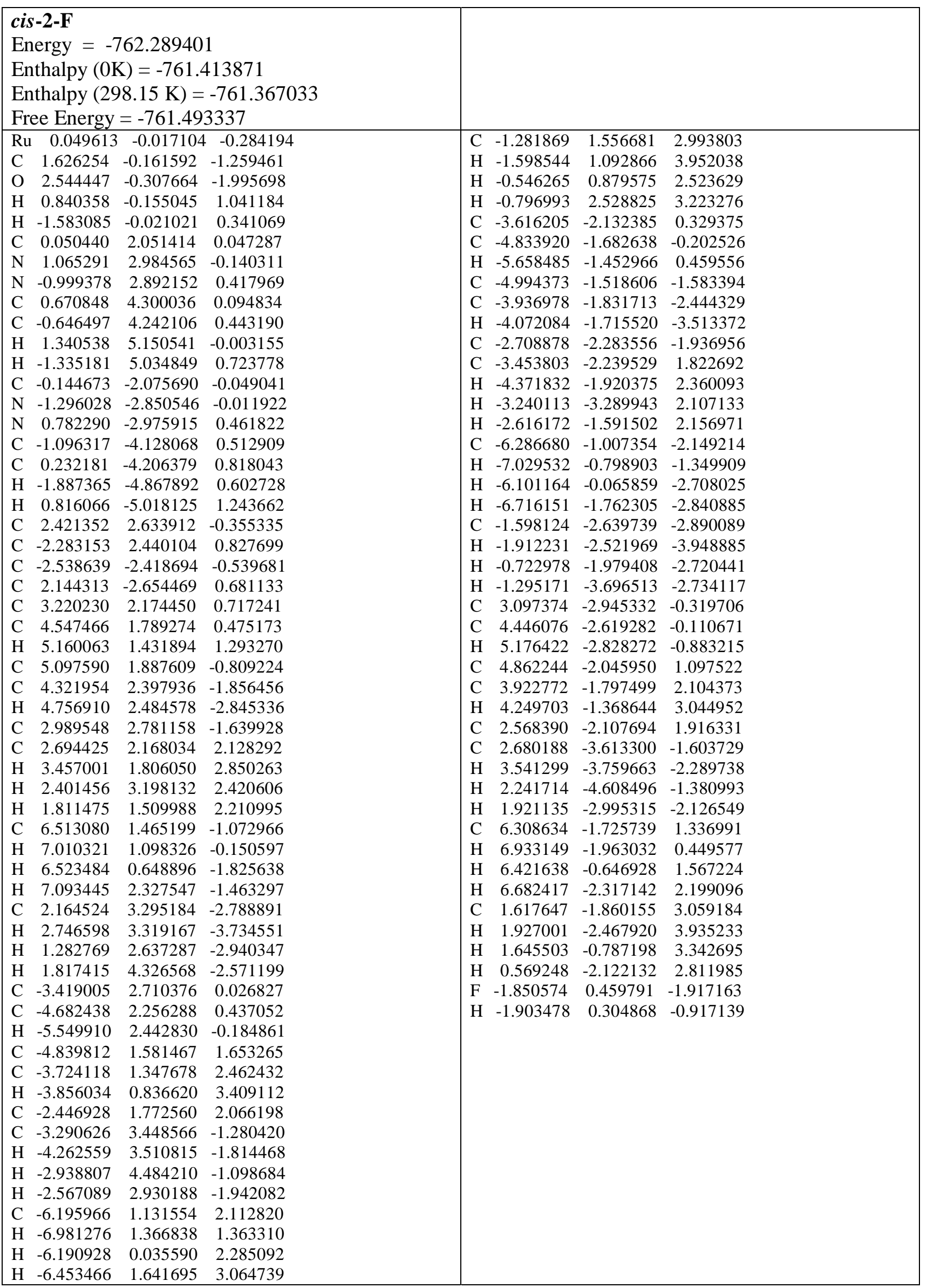




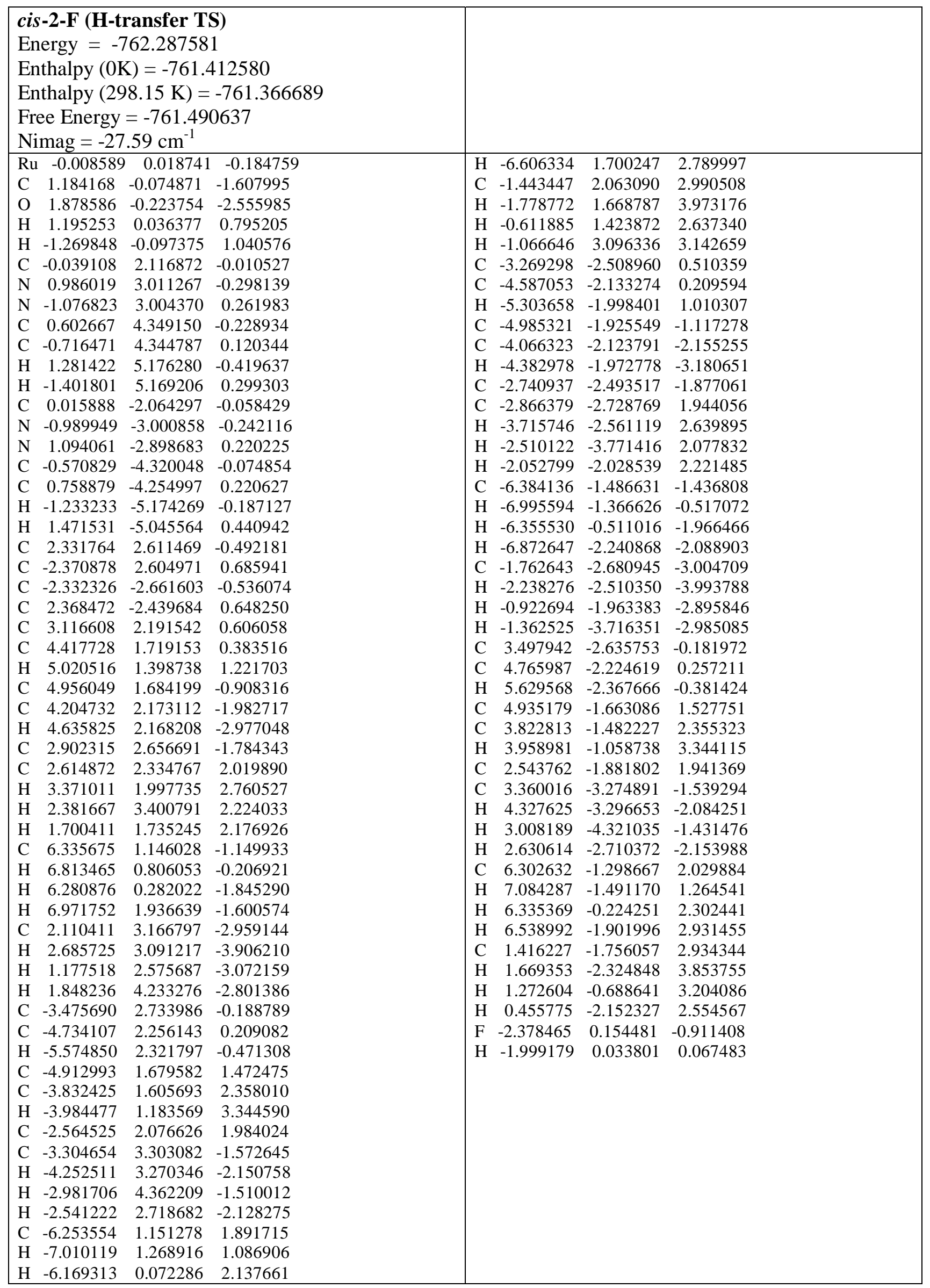




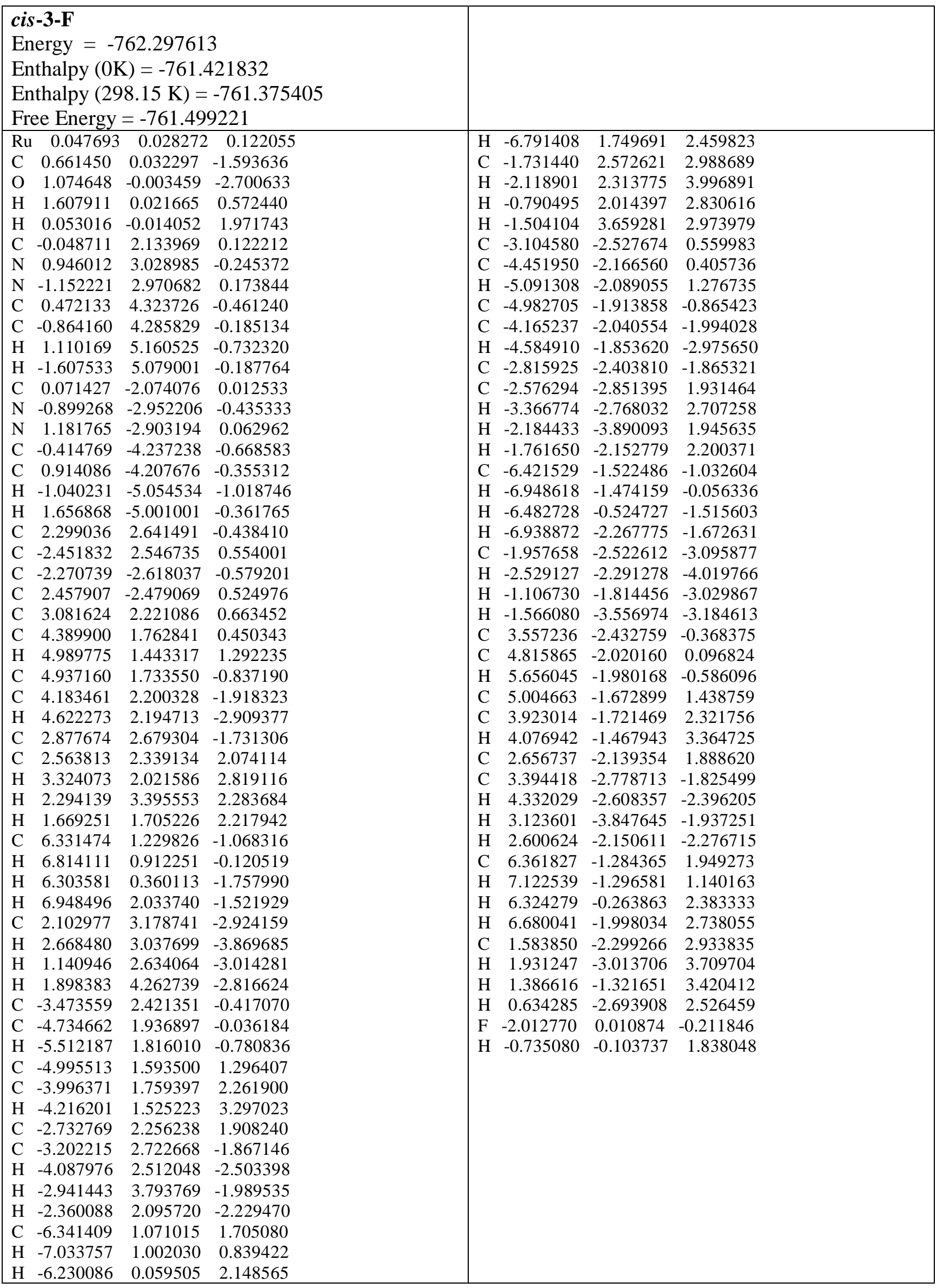




\begin{tabular}{|c|c|c|c|c|c|c|c|}
\hline \multicolumn{4}{|c|}{$\begin{array}{l}\text { cis-3-F }\left(\mathbf{H}_{2} \text { loss } \mathbf{T S}\right) \\
\text { Energy }=-762.294583 \\
\text { Enthalpy }(0 \mathrm{~K})=-761.422309 \\
\text { Enthalpy }(298.15 \mathrm{~K})=-761.375451 \\
\text { Free Energy }=-761.500611 \\
\text { Nimag }=-220.49 \mathrm{~cm}^{-1}\end{array}$} & \multirow{2}{*}{\multicolumn{4}{|c|}{$\begin{array}{llll}\mathrm{H} & 6.807583 & 0.495902 & -1.240930\end{array}$}} \\
\hline & -0.046827 & $\begin{array}{ll}7 & 0.017179\end{array}$ & -0.130282 & & & & \\
\hline & -0.807787 & -0.017823 & 1.492849 & $\mathrm{H}$ & 7.287286 & 2.215753 & -0.948293 \\
\hline & -1.317637 & -0.055980 & 2.567691 & $\mathrm{C}$ & 2.568633 & 2.996521 & -2.916499 \\
\hline & -1.581114 & 0.096606 & -0.566434 & $\mathrm{H}$ & 3.256598 & 2.936275 & -3.786438 \\
\hline & 0.064063 & 0.215411 & -2.342020 & $\mathrm{H}$ & 1.697893 & 2.337590 & -3.117433 \\
\hline & 0.090011 & 2.111012 & -0.148928 & $\mathrm{H}$ & 2.220212 & 4.046029 & -2.828832 \\
\hline & -0.933572 & 3.037692 & -0.032866 & $\mathrm{C}$ & 3.225778 & -2.643839 & -0.751509 \\
\hline $\mathrm{N}$ & 1.172923 & 2.944212 & -0.388349 & $\mathrm{C}$ & 4.554658 & -2.334405 & -0.421559 \\
\hline & -0.508886 & 4.359529 & -0.146916 & $\mathrm{H}$ & 5.306146 & -2.286220 & -1.200602 \\
\hline & 0.833449 & 4.298540 & -0.387112 & $\mathrm{C}$ & 4.922309 & -2.099329 & 0.909803 \\
\hline & -1.180643 & 5.210851 & -0.075926 & $\mathrm{C}$ & 3.954638 & -2.169991 & 1.917700 \\
\hline $\mathrm{H}$ & 1.558477 & 5.093582 & -0.543210 & $\mathrm{H}$ & 4.249341 & -2.003274 & 2.947301 \\
\hline & -0.109325 & -2.074581 & -0.205889 & $\mathrm{C}$ & 2.620034 & -2.473578 & 1.610530 \\
\hline & 0.903545 & -3.008670 & -0.062902 & $\mathrm{C}$ & 2.851967 & -2.906293 & -2.185426 \\
\hline $\mathrm{N}$ & -1.216224 & -2.899082 & -0.329978 & $\mathrm{H}$ & 3.729186 & -2.820585 & -2.861269 \\
\hline C & 0.443541 & -4.324230 & -0.062657 & $\mathrm{H}$ & 2.437609 & -3.931526 & -2.282853 \\
\hline & -0.908475 & -4.257193 & -0.238433 & $\mathrm{H}$ & 2.086856 & -2.172696 & -2.514804 \\
\hline $\mathrm{H}$ & 1.100247 & -5.182940 & 0.052082 & $\mathrm{C}$ & 6.346547 & -1.800090 & 1.273543 \\
\hline $\mathrm{H}$ & -1.649585 & -5.046717 & -0.331985 & $\mathrm{H}$ & 6.998794 & -1.757987 & 0.375490 \\
\hline C & -2.257885 & 2.677076 & 0.316582 & $\mathrm{H}$ & 6.400340 & -0.823368 & 1.797131 \\
\hline C & 2.526077 & 2.516409 & -0.425041 & $\mathrm{H}$ & 6.731125 & -2.591098 & 1.951107 \\
\hline C & 2.244809 & -2.683756 & 0.264316 & & 1.617944 & -2.616086 & 2.723713 \\
\hline C & -2.534062 & -2.422168 & -0.560391 & & 2.079103 & -2.438383 & 3.718391 \\
\hline $\mathrm{C}$ & -3.167379 & 2.271062 & -0.683805 & & 0.799465 & -1.884834 & 2.591482 \\
\hline C & -4.458065 & 1.861223 & -0.319506 & & 1.193475 & -3.641742 & 2.717936 \\
\hline $\mathrm{H}$ & -5.156666 & 1.551063 & -1.084927 & & -3.514566 & -2.544383 & 0.453666 \\
\hline C & -4.855681 & 1.861520 & 1.023812 & C & -4.830361 & -2.123338 & 0.205066 \\
\hline C & -3.967482 & 2.311097 & 2.007460 & $\mathrm{H}$ & -5.580609 & -2.213416 & 0.981653 \\
\hline $\mathrm{H}$ & -4.288019 & 2.330967 & 3.042571 & $\mathrm{C}$ & -5.194440 & -1.617820 & -1.047962 \\
\hline C & -2.677187 & 2.741771 & 1.664172 & C & -4.227082 & -1.495697 & -2.049551 \\
\hline C & -2.779061 & 2.309422 & -2.138199 & $\mathrm{H}$ & -4.514003 & -1.110373 & -3.021606 \\
\hline $\mathrm{H}$ & -3.606172 & 1.963112 & -2.793800 & $\mathrm{C}$ & -2.903637 & -1.902455 & -1.827046 \\
\hline $\mathrm{H}$ & -2.518555 & 3.349628 & -2.425614 & $\mathrm{C}$ & -3.164033 & -3.102369 & 1.808452 \\
\hline $\mathrm{H}$ & -1.901905 & 1.657809 & -2.319922 & $\mathrm{H}$ & -4.028009 & -3.063708 & 2.505409 \\
\hline C & -6.228384 & 1.402976 & 1.419469 & $\mathrm{H}$ & -2.852975 & -4.162503 & 1.714556 \\
\hline $\mathrm{H}$ & -6.829564 & 1.100505 & 0.537041 & $\mathrm{H}$ & -2.334251 & -2.518612 & 2.255779 \\
\hline $\mathrm{H}$ & -6.146836 & 0.532818 & 2.104445 & $\mathrm{C}$ & -6.619261 & -1.247993 & -1.342985 \\
\hline $\mathrm{H}$ & -6.762064 & 2.226614 & 1.938695 & $\mathrm{H}$ & -7.270723 & -1.387369 & -0.454182 \\
\hline C & -1.751590 & 3.253482 & 2.736860 & $\mathrm{H}$ & -6.679053 & -0.187182 & -1.660530 \\
\hline $\mathrm{H}$ & -2.220637 & 3.202826 & 3.742327 & $\mathrm{H}$ & -7.005392 & -1.887503 & -2.164335 \\
\hline $\mathrm{H}$ & -0.820183 & 2.650850 & 2.758291 & $\mathrm{C}$ & -1.948785 & -1.836861 & -2.989809 \\
\hline $\mathrm{H}$ & -1.490912 & 4.312544 & 2.532545 & $\mathrm{H}$ & -2.339708 & -2.459213 & -3.822136 \\
\hline C & 3.181193 & 2.098963 & 0.759171 & $\mathrm{H}$ & -1.866646 & -0.788076 & -3.342828 \\
\hline C & 4.532736 & 1.722002 & 0.706827 & $\mathrm{H}$ & -0.934726 & -2.207101 & -2.743875 \\
\hline $\mathrm{H}$ & 5.037393 & 1.401092 & 1.610160 & F & 1.991380 & -0.106162 & -0.482148 \\
\hline C & 5.238912 & 1.759261 & -0.502379 & $\mathrm{H}$ & 0.779389 & 0.008111 & -2.155185 \\
\hline C & 4.590984 & 2.177866 & -1.669055 & & & & \\
\hline $\mathrm{H}$ & 5.142932 & 2.202908 & -2.601511 & & & & \\
\hline C & 3.244666 & 2.569846 & -1.640609 & & & & \\
\hline C & 2.456493 & 2.072908 & 2.078295 & & & & \\
\hline $\mathrm{H}$ & 3.126956 & 1.768571 & 2.909870 & & & & \\
\hline $\mathrm{H}$ & 2.055428 & 3.082560 & 2.307481 & & & & \\
\hline $\mathrm{H}$ & 1.621109 & 1.346226 & 2.035598 & & & & \\
\hline C & 6.685737 & 1.366156 & -0.562062 & & & & \\
\hline $\mathrm{H}$ & 7.078121 & 1.092404 & 0.439289 & & & & \\
\hline
\end{tabular}




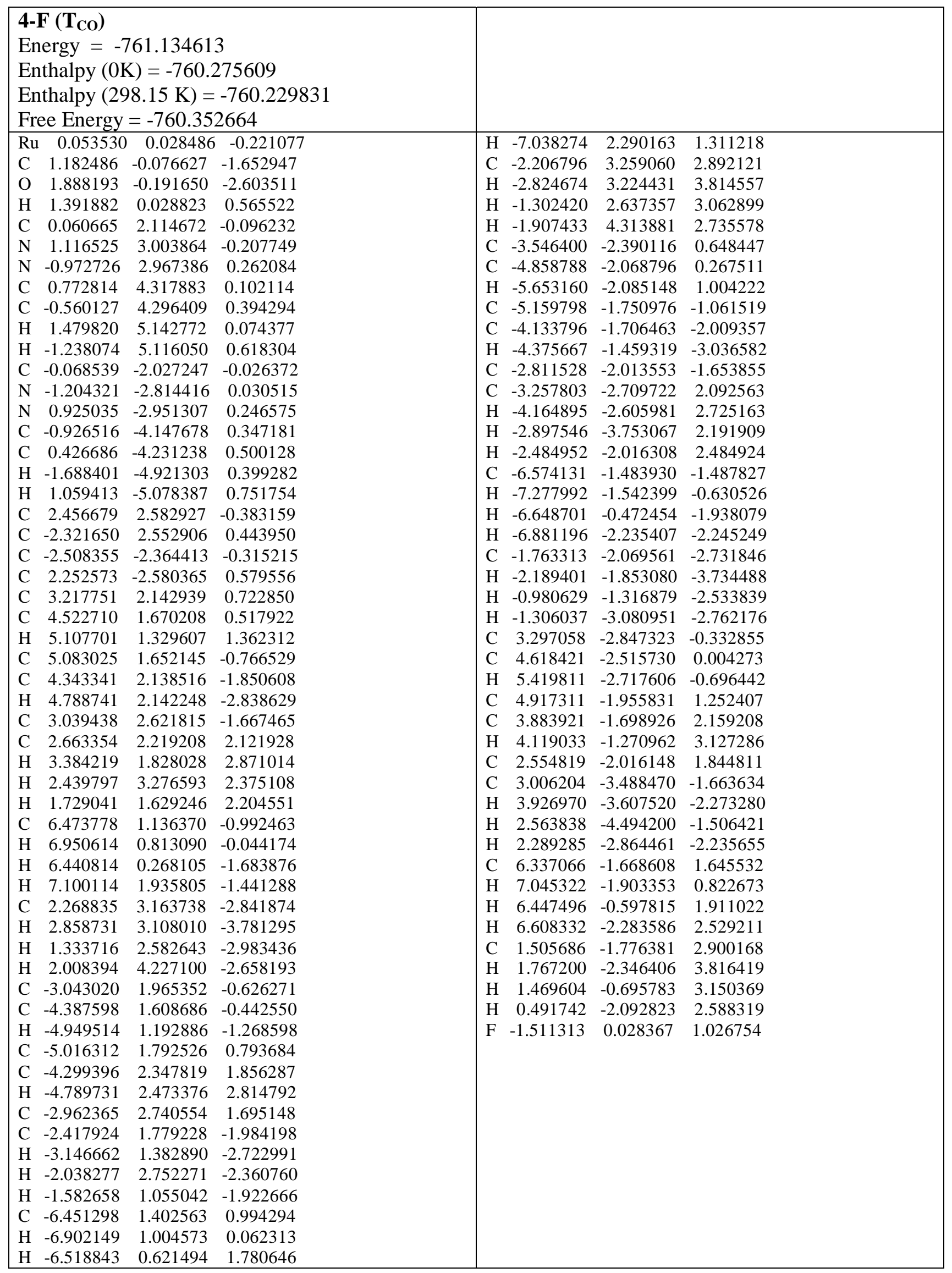




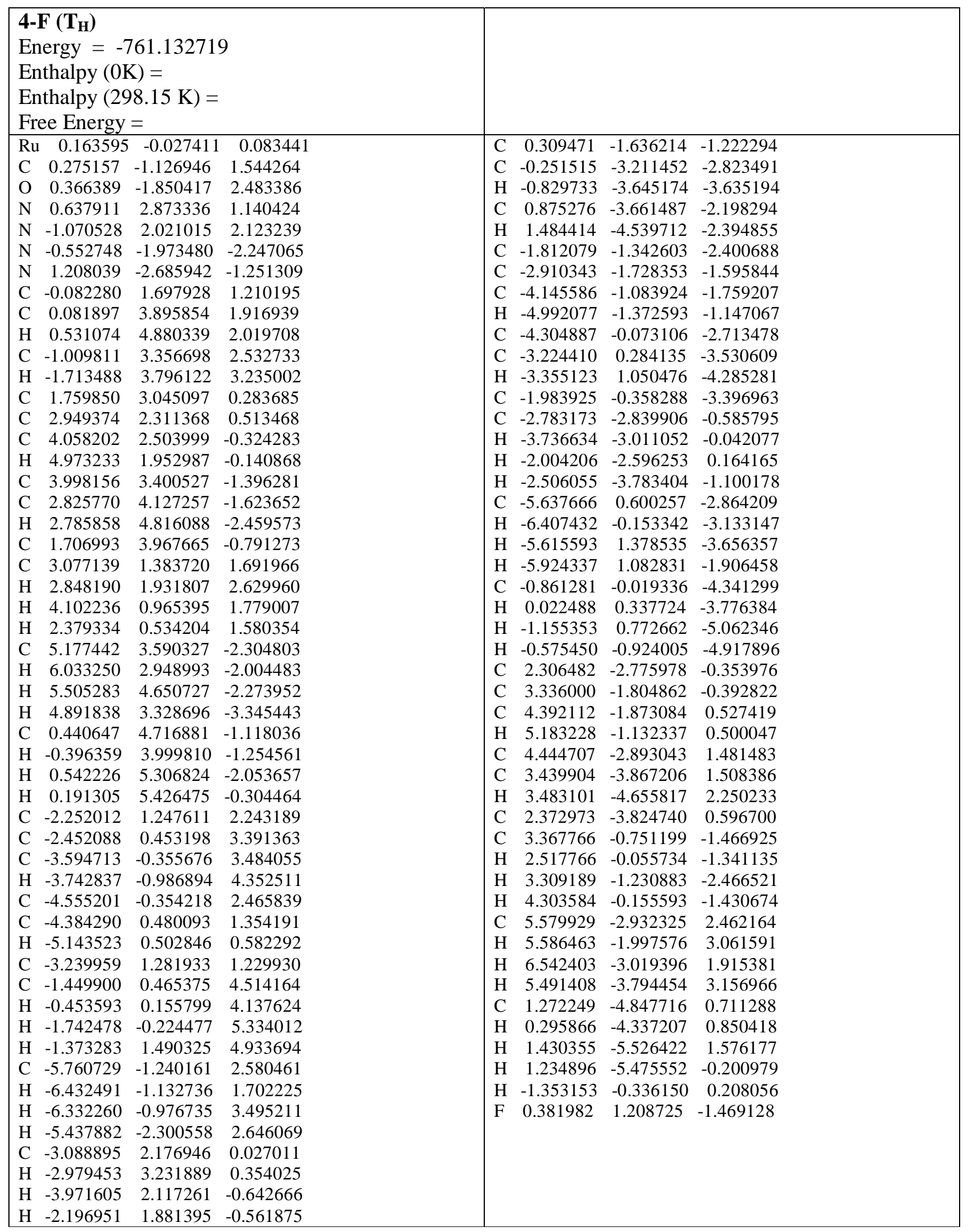




\begin{tabular}{|c|c|c|c|c|c|c|}
\hline & $\begin{array}{l}-\mathbf{- 2}-\mathbf{N H}_{2} \\
\text { ergy = - } \\
\text { thalpy }(0 \\
\text { thalpy }(2 \\
\text { ee Energy }\end{array}$ & $\begin{array}{l}718.416932 \\
K)=-717.514277 \\
8.15 \mathrm{~K})=-717.467115 \\
=-717.592293\end{array}$ & & & & \\
\hline & -0.08990 & $0.065396-0.331575$ & $\mathrm{H}$ & 6.496219 & -0.488703 & 2.355576 \\
\hline & -1.793999 & $0.158711-1.109828$ & & 6.623789 & -2.232207 & 2.803698 \\
\hline & -2.787567 & $0.259983 \quad-1.762564$ & & 1.491271 & -1.869701 & 2.786504 \\
\hline & -0.583498 & $0.140242 \quad 1.171823$ & $\mathrm{H}$ & 1.831199 & -1.610851 & 3.811701 \\
\hline & 1.515640 & $0.037637 \quad 0.185718$ & $\mathrm{H}$ & 0.772298 & -1.094279 & 2.470289 \\
\hline & 0.039268 & $-1.999280-0.116752$ & $\mathrm{H}$ & 0.981477 & -2.855003 & 2.831009 \\
\hline & -0.943569 & $\begin{array}{ll}-2.984979 & -0.253478\end{array}$ & $\mathrm{C}$ & 3.215411 & 2.002508 & 0.693161 \\
\hline & 1.175552 & $-2.808523 \quad 0.038266$ & $\mathrm{C}$ & 4.525601 & 1.540492 & 0.502527 \\
\hline & -0.457131 & $-4.289627 \quad-0.166743$ & $\mathrm{H}$ & 5.091329 & 1.173540 & 1.347833 \\
\hline & 0.886244 & $-4.178507 \quad 0.026992$ & $\mathrm{C}$ & 5.114086 & 1.559592 & -0.768144 \\
\hline & -1.090503 & $-5.170227-0.242000$ & $\mathrm{C}$ & 4.403155 & 2.089055 & -1.850496 \\
\hline & 1.642023 & $-4.948629 \quad 0.162705$ & $\mathrm{H}$ & 4.873479 & 2.128157 & -2.826134 \\
\hline & 0.061327 & $2.123719-0.107425$ & $\mathrm{C}$ & 3.102912 & 2.586150 & -1.677726 \\
\hline & 1.160959 & $2.969749 \quad-0.241676$ & $\mathrm{C}$ & 2.621531 & 1.999649 & 2.077770 \\
\hline & -0.925161 & $3.055766 \quad 0.220704$ & & 3.337166 & 1.606557 & 2.830847 \\
\hline & 0.875035 & $4.315224 \quad 0.006865$ & $\mathrm{H}$ & 2.345799 & 3.034771 & 2.368860 \\
\hline & -0.456633 & $4.368668 \quad 0.296345$ & & 1.716181 & 1.361546 & 2.107235 \\
\hline & 1.621940 & $5.103519-0.041951$ & & 6.505336 & 1.038739 & -0.978469 \\
\hline & -1.090375 & $5.214030 \quad 0.552489$ & $\mathrm{H}$ & 6.951684 & 0.669558 & -0.031586 \\
\hline & -2.332179 & $-2.707955 \quad-0.329600$ & $\mathrm{H}$ & 6.482558 & 0.200461 & -1.706279 \\
\hline $\mathrm{C}$ & 2.446170 & $-2.342026 \quad 0.473549$ & $\mathrm{H}$ & 7.151444 & 1.849136 & -1.376644 \\
\hline & 2.483964 & $2.494844 \quad-0.412020$ & & 2.383692 & 3.213692 & -2.841997 \\
\hline & -2.247348 & $2.698938 \quad 0.591804$ & $\mathrm{H}$ & 2.992740 & 3.176857 & -3.770071 \\
\hline $\mathrm{C}$ & -3.053095 & $-2.329194 \quad 0.827151$ & $\mathrm{H}$ & 1.428899 & 2.683901 & -3.031378 \\
\hline $\mathrm{C}$ & -4.421056 & -2.033180 & & 2.164382 & 4.277827 & -2.615562 \\
\hline $\mathrm{H}$ & -4.974256 & $-1.736578 \quad 1.607120$ & & -3.315863 & 2.956204 & -0.298078 \\
\hline $\mathrm{C}$ & -5.085725 & $-2.140404-0.503500$ & $\mathrm{C}$ & -4.614972 & 2.545075 & 0.038144 \\
\hline $\mathrm{C}$ & -4.382404 & $-2.567421-1.635136$ & $\mathrm{H}$ & -5.430717 & 2.718743 & -0.653486 \\
\hline $\mathrm{H}$ & -4.904808 & $\begin{array}{ll}-2.659698 & -2.580230\end{array}$ & $\mathrm{C}$ & -4.869394 & 1.911224 & 1.260261 \\
\hline $\mathrm{C}$ & -3.012663 & $-2.862301 \quad-1.558727$ & & -3.821265 & 1.706746 & 2.163342 \\
\hline $\mathrm{C}$ & -2.396434 & $-2.316821 \quad 2.181353$ & $\mathrm{H}$ & -4.027572 & 1.248259 & 3.124009 \\
\hline $\mathrm{H}$ & -3.114279 & -2.047301 & & -2.516181 & 2.115957 & 1.855365 \\
\hline $\mathrm{H}$ & -1.985816 & $-3.323394 \quad 2.406663$ & $\mathrm{C}$ & -3.069080 & 3.624656 & -1.625082 \\
\hline $\mathrm{H}$ & -1.575363 & $-1.580246 \quad 2.203911$ & $\mathrm{H}$ & -4.001352 & 3.708779 & -2.222928 \\
\hline $\mathrm{C}$ & -6.546419 & $-1.816149-0.617611$ & $\mathrm{H}$ & -2.672724 & 4.648196 & -1.463384 \\
\hline $\mathrm{H}$ & -6.976791 & $-1.511930 \quad 0.359566$ & $\mathrm{H}$ & -2.332581 & 3.037689 & -2.213021 \\
\hline $\mathrm{H}$ & -6.687357 & $-0.983195 \quad-1.338249$ & $\mathrm{C}$ & -6.258674 & 1.478427 & 1.626330 \\
\hline $\mathrm{H}$ & -7.099589 & $-2.708174 \quad-0.979751$ & $\mathrm{H}$ & -6.983000 & 1.693736 & 0.812200 \\
\hline $\mathrm{C}$ & -2.272628 & $-3.284551 \quad-2.799619$ & $\mathrm{H}$ & -6.269959 & 0.387767 & 1.826732 \\
\hline $\mathrm{H}$ & -2.941320 & $-3.318213 \quad-3.685829$ & $\mathrm{H}$ & -6.586838 & 2.015166 & 2.541173 \\
\hline $\mathrm{H}$ & -1.454520 & $-2.564428 \quad-3.010467$ & $\mathrm{C}$ & -1.469293 & 1.996794 & 2.932542 \\
\hline $\mathrm{H}$ & -1.841337 & $-4.296760 \quad-2.656530$ & $\mathrm{H}$ & -1.786392 & 2.585477 & 3.818935 \\
\hline C & 3.568608 & $-2.476382 \quad-0.377656$ & $\mathrm{H}$ & -1.358850 & 0.935312 & 3.238293 \\
\hline $\mathrm{C}$ & 4.851284 & $-2.172755 \quad 0.103971$ & $\mathrm{H}$ & -0.480652 & 2.380021 & 2.613634 \\
\hline $\mathrm{H}$ & 5.709854 & $-2.274000 \quad-0.549174$ & $\mathrm{~N}$ & 0.801208 & -0.090753 & -2.467084 \\
\hline C & 5.039900 & $\begin{array}{ll}-1.774191 & 1.431789\end{array}$ & $\mathrm{H}$ & 0.646173 & -1.038959 & -2.819628 \\
\hline C & 3.932787 & $-1.636864 \quad 2.274203$ & $\mathrm{H}$ & 1.808064 & 0.086330 & -2.479445 \\
\hline $\mathrm{H}$ & 4.087530 & $-1.330898 \quad 3.302380$ & $\mathrm{H}$ & 0.349459 & 0.553591 & -3.121590 \\
\hline $\mathrm{C}$ & 2.638337 & $-1.926750 \quad 1.814945$ & & & & \\
\hline C & 3.406905 & $-2.938416 \quad-1.801504$ & & & & \\
\hline $\mathrm{H}$ & 4.377619 & $-2.956643 \quad-2.341068$ & & & & \\
\hline $\mathrm{H}$ & 2.985440 & $\begin{array}{ll}-3.964097 & -1.824474\end{array}$ & & & & \\
\hline $\mathrm{H}$ & 2.726419 & $\begin{array}{ll}-2.252680 & -2.343662\end{array}$ & & & & \\
\hline $\mathrm{C}$ & 6.419143 & $-1.526620 \quad 1.971252$ & & & & \\
\hline $\mathrm{H}$ & 7.195907 & $-1.671671 \quad 1.190650$ & & & & \\
\hline
\end{tabular}




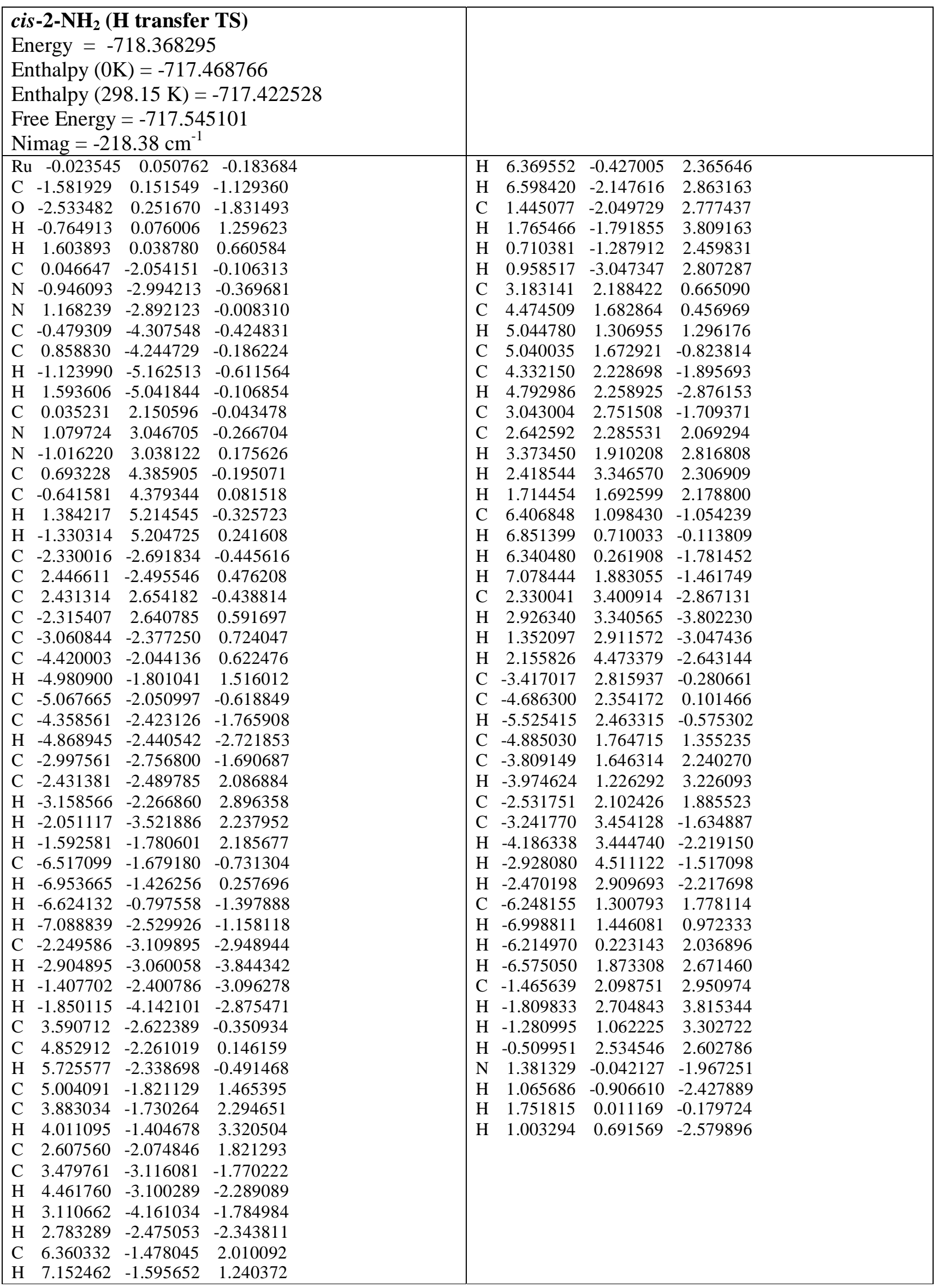




\begin{tabular}{|c|c|c|c|c|c|c|}
\hline \multicolumn{3}{|c|}{$\begin{array}{l}\text { cis-3-NH } \\
\text { Energy }=-718.370656 \\
\text { Enthalpy }(0 \mathrm{~K})=-717.470556 \\
\text { Enthalpy }(298.15 \mathrm{~K})=-717.423772 \\
\text { Free Energy }=-717.548546\end{array}$} & \multirow{2}{*}{\multicolumn{4}{|c|}{$\begin{array}{llll}\mathrm{H} & 6.047392 & -0.083179 & 2.748792\end{array}$}} \\
\hline \multicolumn{3}{|c|}{$\begin{array}{llll}\mathrm{Ru} & -0.046080 & 0.043432 & -0.023610\end{array}$} & & & & \\
\hline & -1.446910 & $0.173323-1.202804$ & \multicolumn{4}{|c|}{$\begin{array}{llll}\text { H } & 6.492009 & -1.789010 & 3.146534\end{array}$} \\
\hline & -2.319417 & $0.316215-1.988733$ & \multicolumn{4}{|c|}{$\begin{array}{llll}\text { C } & 1.389632 & -2.404173 & 2.884226\end{array}$} \\
\hline & -1.083602 & $-0.029937 \quad 1.242242$ & \multirow{2}{*}{\multicolumn{4}{|c|}{$\begin{array}{llll}\mathrm{H} & 1.655330 & -2.211142 & 3.945252 \\
\mathrm{H} & 0.535338 & -1.752230 & 2633840\end{array}$}} \\
\hline & 0.935329 & $0.110426 \quad 1.445547$ & & & & \\
\hline & 0.084673 & $-2.058613-0.051172$ & \multicolumn{4}{|c|}{$\begin{array}{llll}\mathrm{H} & 0.07590 & -1.152250 & 2.053840 \\
\mathrm{H} & 1.075939 & -3.465642 & 2.796862\end{array}$} \\
\hline & -0.863824 & $-3.005765 \quad-0.414677$ & \multicolumn{4}{|c|}{$\begin{array}{llll}\text { C } & 3.272624 & 2.177532 & 0.465875\end{array}$} \\
\hline & 1.237175 & $-2.851352 \quad 0.002228$ & \multicolumn{4}{|c|}{$\begin{array}{llll}C & 5.25024 & 2.171552 & 0.405015 \\
\mathrm{C} & 4.500161 & 1.627407 & 0.069699\end{array}$} \\
\hline & -0.340005 & $-4.288096-0.583681$ & \multicolumn{4}{|c|}{$\begin{array}{llll}\mathrm{H} & 5.192823 & 1.264414 & 0.817267\end{array}$} \\
\hline & 0.989433 & $-4.194113-0.300090$ & \multicolumn{4}{|c|}{$\begin{array}{llll}\text { C } & 4.856266 & 1.576096 & -1.283387\end{array}$} \\
\hline & -0.943363 & $-5.148211-0.861939$ & \multicolumn{4}{|c|}{$\begin{array}{llll}\text { C } & 4.006429 & 2.138917 & -2.242855\end{array}$} \\
\hline & 1.753692 & $-4.966193-0.268470$ & \multicolumn{4}{|c|}{$\begin{array}{llll}\mathrm{H} & 4.308774 & 2.139758 & -3.283576\end{array}$} \\
\hline & 0.019173 & $2.142908 \quad 0.088837$ & \multicolumn{4}{|c|}{$\begin{array}{llll}\text { C } & 2.774444 & 2.698442 & -1.868664\end{array}$} \\
\hline & 1.059804 & $3.038002-0.140005$ & \multicolumn{4}{|c|}{$\begin{array}{llll}\text { C } & 2.989821 & 2.383719 & 1.932326\end{array}$} \\
\hline & -1.035286 & $3.022670 \quad 0.307350$ & \multicolumn{4}{|c|}{ Н $\quad 3.802450 \quad 1.972652 \quad 2.568505$} \\
\hline & 0.657592 & $4.374311-0.114308$ & \multicolumn{4}{|c|}{$\begin{array}{llll}\mathrm{H} & 2.904781 & 3.471312 & 2.138153\end{array}$} \\
\hline & -0.675030 & $4.364678 \quad 0.177196$ & \multicolumn{4}{|c|}{$\begin{array}{llll}\mathrm{H} & 2.041957 & 1.901535 & 2.233121\end{array}$} \\
\hline & 1.341606 & $5.204696-0.269337$ & & 6.153228 & 0.954635 & -1.711093 \\
\hline & -1.365147 & $5.189032 \quad 0.336196$ & & 6.725300 & 0.563415 & -0.842844 \\
\hline & -2.244991 & $-2.716414-0.566871$ & $\mathrm{H}$ & 5.949732 & 0.111162 & -2.404105 \\
\hline $\mathrm{C}$ & 2.470469 & $-2.432702 \quad 0.575301$ & $\mathrm{H}$ & 6.777139 & 1.711433 & -2.231406 \\
\hline & 2.365262 & $2.631895-0.516368$ & & 1.898630 & 3.336550 & -2.916158 \\
\hline & -2.327491 & $2.605763 \quad 0.724953$ & $\mathrm{H}$ & 2.338743 & 3.242188 & -3.931521 \\
\hline & -3.050499 & $-2.455098 \quad 0.566307$ & $\mathrm{H}$ & 0.895802 & 2.862449 & -2.927225 \\
\hline & -4.393546 & -2.089975 & & 1.780893 & 4.417387 & -2.695733 \\
\hline $\mathrm{H}$ & -5.010048 & $-1.884898 \quad 1.258262$ & $\mathrm{C}$ & -3.435665 & 2.770397 & -0.141319 \\
\hline $\mathrm{C}$ & -4.954274 & $-2.012985-0.887358$ & & -4.691455 & 2.268781 & 0.235028 \\
\hline $\mathrm{C}$ & -4.177191 & $-2.343941 \quad-2.002599$ & & -5.534924 & 2.368534 & -0.437806 \\
\hline $\mathrm{H}$ & -4.622275 & $-2.299498 \quad-2.989786$ & $\mathrm{C}$ & -4.871818 & 1.651011 & 1.477917 \\
\hline $\mathrm{C}$ & -2.829226 & $-2.707433-1.855633$ & & -3.791146 & 1.544833 & 2.358947 \\
\hline $\mathrm{C}$ & -2.521253 & $-2.662413 \quad 1.960111$ & & -3.941916 & 1.098199 & 3.335345 \\
\hline & -3.303200 & -2.484327 & & -2.525538 & 2.035764 & 2.008060 \\
\hline $\mathrm{H}$ & -2.159457 & -3.706301 & $\mathrm{C}$ & -3.284038 & 3.443963 & -1.481319 \\
\hline $\mathrm{H}$ & -1.688712 & $-1.969923 \quad 2.165130$ & $\mathrm{H}$ & -4.227538 & 3.412279 & -2.066535 \\
\hline $\mathrm{C}$ & -6.382108 & $-1.591129-1.074694$ & & -3.011394 & 4.509269 & -1.339817 \\
\hline $\mathrm{H}$ & -6.873693 & $-1.366885 \quad-0.104235$ & $\mathrm{H}$ & -2.492356 & 2.944018 & -2.076099 \\
\hline $\mathrm{H}$ & -6.419961 & $-0.677500 \quad-1.704716$ & $\mathrm{C}$ & -6.220300 & 1.137703 & 1.891163 \\
\hline $\mathrm{H}$ & -6.949016 & $-2.403283-1.576482$ & $\mathrm{H}$ & -6.976838 & 1.283181 & 1.090925 \\
\hline $\mathrm{C}$ & -2.005232 & $\begin{array}{ll}-3.003709 & -3.080957\end{array}$ & $\mathrm{H}$ & -6.156573 & 0.054410 & 2.120126 \\
\hline $\mathrm{H}$ & -2.598368 & $-2.887882-4.012846$ & $\mathrm{H}$ & -6.561072 & 1.677043 & 2.799849 \\
\hline $\mathrm{H}$ & -1.142540 & $-2.306385 \quad-3.131923$ & $\mathrm{C}$ & -1.443493 & 2.007083 & 3.058650 \\
\hline $\mathrm{H}$ & -1.631606 & $-4.047240 \quad-3.043811$ & $\mathrm{H}$ & -1.781208 & 2.579655 & 3.948027 \\
\hline $\mathrm{C}$ & 3.654346 & $-2.407366 \quad-0.205322$ & $\mathrm{H}$ & -1.243508 & 0.960550 & 3.370396 \\
\hline $\mathrm{C}$ & 4.852805 & $-1.952181 \quad 0.367421$ & $\mathrm{H}$ & -0.493489 & 2.460110 & 2.714124 \\
\hline $\mathrm{H}$ & 5.752391 & $-1.901399-0.234300$ & $\mathrm{~N}$ & 1.398014 & -0.056463 & -1.715489 \\
\hline $\mathrm{C}$ & 4.907384 & $-1.579782 \quad 1.714858$ & $\mathrm{H}$ & 1.137334 & -0.951673 & -2.158173 \\
\hline $\mathrm{C}$ & 3.761835 & $-1.689163 \quad 2.506884$ & $\mathrm{H}$ & 1.524819 & 0.088990 & 0.833457 \\
\hline $\mathrm{H}$ & 3.823584 & $-1.443814 \quad 3.560812$ & $\mathrm{H}$ & 1.019963 & 0.600956 & -2.415469 \\
\hline $\mathrm{C}$ & 2.550931 & $-2.140410 \quad 1.960772$ & & & & \\
\hline $\mathrm{C}$ & 3.663767 & $-2.862025-1.642276$ & & & & \\
\hline $\mathrm{H}$ & 4.667152 & $-2.743514 \quad-2.103911$ & & & & \\
\hline $\mathrm{H}$ & 3.393211 & $\begin{array}{ll}-3.935087 & -1.703816\end{array}$ & & & & \\
\hline $\mathrm{H}$ & 2.948747 & $-2.272210 \quad-2.243004$ & & & & \\
\hline $\mathrm{C}$ & 6.190580 & $-1.100964 \quad 2.328914$ & & & & \\
\hline $\mathrm{H}$ & 7.011591 & $-1.060217 \quad 1.581779$ & & & & \\
\hline
\end{tabular}




\begin{tabular}{|c|c|c|c|c|c|c|c|}
\hline \multicolumn{4}{|c|}{$\begin{array}{l}\mathbf{4 - N H}\left(\mathbf{T}_{\mathbf{C o}}\right) \\
\text { Energy }=-717.200301 \\
\text { Enthalpy }(0 \mathrm{~K})=-716.317376 \\
\text { Enthalpy }(298.15 \mathrm{~K})=-716.271151 \\
\text { Free Energy }=-716.395759\end{array}$} & & \multirow[b]{2}{*}{-2.712478} & \multirow{2}{*}{\multicolumn{2}{|c|}{$4.762512-1.583184$}} \\
\hline \multicolumn{4}{|c|}{$\begin{array}{llll}\mathrm{Ru} & -0.080982 & -0.048722 & -0.196052\end{array}$} & & & & \\
\hline & 1.193442 & $-0.470051-$ & -1.439395 & $\mathrm{H}$ & -2.830480 & 3.021031 & -2.066631 \\
\hline $\mathrm{O}$ & 1.910487 & $-0.820501-$ & -2.323496 & & -5.689291 & 2.742403 & 2.843903 \\
\hline $\mathrm{H}$ & 1.180782 & 0.105398 & 0.746731 & $\mathrm{H}$ & -6.563118 & 3.068394 & 2.240526 \\
\hline $\mathrm{C}$ & 0.103676 & 2.053651 & -0.385753 & $\mathrm{H}$ & -5.851107 & 1.688714 & 3.154689 \\
\hline & 1.182055 & 2.764178 & -0.928892 & $\mathrm{H}$ & -5.629378 & 3.382637 & 3.749143 \\
\hline $\mathrm{N}$ & -0.775418 & 3.111646 & -0.112354 & $\mathrm{C}$ & -0.713420 & 2.220950 & 2.673671 \\
\hline & 0.981121 & 4.142951 & -0.971548 & $\mathrm{H}$ & -0.886721 & 1.956886 & 3.738519 \\
\hline & -0.259516 & 4.364363 & -0.461256 & $\mathrm{H}$ & -0.242108 & 1.349748 & 2.184896 \\
\hline & 1.720998 & 4.841055 & -1.355294 & $\mathrm{H}$ & -0.024222 & 3.090722 & 2.640589 \\
\hline $\mathrm{H}$ & -0.784668 & 5.300299 & -0.286634 & $\mathrm{C}$ & -3.512871 & -3.130963 & 0.060954 \\
\hline & -0.184619 & -2.128295 & 0.071334 & $\mathrm{C}$ & -4.827432 & -2.862443 & -0.351256 \\
\hline $\mathrm{N}$ & -1.089887 & -3.084411 & -0.388160 & $\mathrm{H}$ & -5.656961 & -3.101381 & 0.303695 \\
\hline $\mathrm{N}$ & 0.834992 & -2.939985 & 0.558731 & $\mathrm{C}$ & -5.082286 & -2.282236 & -1.598021 \\
\hline & -0.653801 & -4.394473 & -0.161099 & $\mathrm{C}$ & -4.015177 & -1.969281 & -2.443872 \\
\hline & 0.574968 & -4.302161 & 0.420930 & $\mathrm{H}$ & -4.221759 & -1.527594 & -3.411982 \\
\hline $\mathrm{H}$ & -1.220524 & -5.270027 & -0.468835 & $\mathrm{C}$ & -2.690173 & -2.217145 & -2.053435 \\
\hline $\mathrm{H}$ & 1.245346 & -5.083988 & 0.767646 & $\mathrm{C}$ & -3.283949 & -3.700362 & 1.437841 \\
\hline $\mathrm{C}$ & 2.479725 & 2.218957 & -1.109390 & $\mathrm{H}$ & -4.234830 & -3.809010 & 2.001532 \\
\hline $\mathrm{C}$ & -1.991236 & 3.007998 & 0.619196 & $\mathrm{H}$ & -2.823632 & -4.706015 & 1.368104 \\
\hline $\mathrm{C}$ & -2.425915 & -2.792879 & -0.787320 & $\mathrm{H}$ & -2.618972 & -3.026764 & 2.017514 \\
\hline $\mathrm{C}$ & 1.975948 & -2.429814 & 1.236070 & $\mathrm{C}$ & -6.487594 & -1.991657 & -2.037662 \\
\hline $\mathrm{C}$ & 3.318273 & 1.966101 & 0.002830 & $\mathrm{H}$ & -7.228568 & -2.291952 & -1.266501 \\
\hline $\mathrm{C}$ & 4.567128 & 1.358771 & -0.197968 & $\mathrm{H}$ & -6.600933 & -0.903547 & -2.228008 \\
\hline $\mathrm{H}$ & 5.203617 & 1.142301 & 0.650874 & $\mathrm{H}$ & -6.708685 & -2.548557 & -2.972503 \\
\hline $\mathrm{C}$ & 5.014051 & $1.052384-$ & -1.488635 & $\mathrm{C}$ & -1.579994 & -1.904088 & -3.014322 \\
\hline $\mathrm{C}$ & 4.227361 & 1.399301 & -2.592636 & $\mathrm{H}$ & -1.968898 & -1.595545 & -4.008016 \\
\hline $\mathrm{H}$ & 4.591547 & 1.187360 & -3.591125 & $\mathrm{H}$ & -0.988430 & -1.062606 & -2.618252 \\
\hline $\mathrm{C}$ & 2.969413 & 1.996174 & -2.416042 & $\mathrm{H}$ & -0.933617 & -2.795466 & -3.157854 \\
\hline $\mathrm{C}$ & 2.938618 & 2.425333 & 1.386238 & $\mathrm{C}$ & 3.268172 & -2.595659 & 0.677940 \\
\hline $\mathrm{H}$ & 3.738045 & 2.209110 & 2.125307 & $\mathrm{C}$ & 4.371771 & -1.978474 & 1.287863 \\
\hline $\mathrm{H}$ & 2.765769 & 3.521789 & 1.380618 & $\mathrm{H}$ & 5.356495 & -2.077664 & 0.846541 \\
\hline $\mathrm{H}$ & 2.020943 & 1.919780 & 1.730353 & $\mathrm{C}$ & 4.218330 & -1.239585 & 2.466339 \\
\hline $\mathrm{C}$ & 6.335030 & $0.373129-$ & -1.700636 & $\mathrm{C}$ & 2.953876 & -1.129444 & 3.051563 \\
\hline $\mathrm{H}$ & 6.846068 & 0.162342 & -0.736953 & $\mathrm{H}$ & 2.843484 & -0.584764 & 3.982540 \\
\hline $\mathrm{H}$ & 6.176468 & -0.590255 & -2.229615 & $\mathrm{C}$ & 1.834208 & -1.735045 & 2.464544 \\
\hline $\mathrm{H}$ & 6.994509 & 1.021529 & -2.315014 & $\mathrm{C}$ & 3.482583 & -3.395900 & -0.580754 \\
\hline $\mathrm{C}$ & 2.130766 & 2.338559 & -3.618480 & $\mathrm{H}$ & 4.536359 & -3.342326 & -0.928027 \\
\hline $\mathrm{H}$ & 2.642260 & 2.068382 & -4.566642 & $\mathrm{H}$ & 3.244522 & -4.462524 & -0.395629 \\
\hline $\mathrm{H}$ & 1.167350 & 1.788252 & -3.574715 & $\mathrm{H}$ & 2.834301 & -3.016637 & -1.394434 \\
\hline $\mathrm{H}$ & 1.926442 & 3.429202 & -3.635656 & $\mathrm{C}$ & 5.400354 & -0.590616 & 3.125283 \\
\hline C & -3.213885 & 3.400376 & 0.017016 & $\mathrm{H}$ & 6.334426 & -0.759804 & 2.548379 \\
\hline C & -4.409671 & 3.310209 & 0.746431 & $\mathrm{H}$ & 5.231496 & 0.503305 & 3.204855 \\
\hline $\mathrm{H}$ & -5.347118 & 3.589991 & 0.280535 & $\mathrm{H}$ & 5.535378 & -1.009800 & 4.144496 \\
\hline & -4.409712 & 2.847255 & 2.066617 & $\mathrm{C}$ & 0.529837 & -1.677511 & 3.216870 \\
\hline $\mathrm{C}$ & -3.203657 & 2.478287 & 2.668648 & $\mathrm{H}$ & 0.667838 & -2.123072 & 4.224528 \\
\hline $\mathrm{H}$ & -3.210827 & 2.132872 & 3.696050 & $\mathrm{H}$ & 0.212158 & -0.623646 & 3.339341 \\
\hline $\mathrm{C}$ & -1.993079 & 2.559538 & 1.963640 & $\mathrm{H}$ & -0.284864 & -2.237825 & 2.719562 \\
\hline & -3.270477 & $3.817686-$ & -1.430176 & $\mathrm{~N}$ & -2.062262 & 0.014647 & 0.197010 \\
\hline & -4.314579 & 3.988898 & -1.768325 & & -2.771525 & 0.686556 & -0.100296 \\
\hline & & & & $\mathrm{H}$ & -2.529996 & -0.660797 & 0.806083 \\
\hline
\end{tabular}




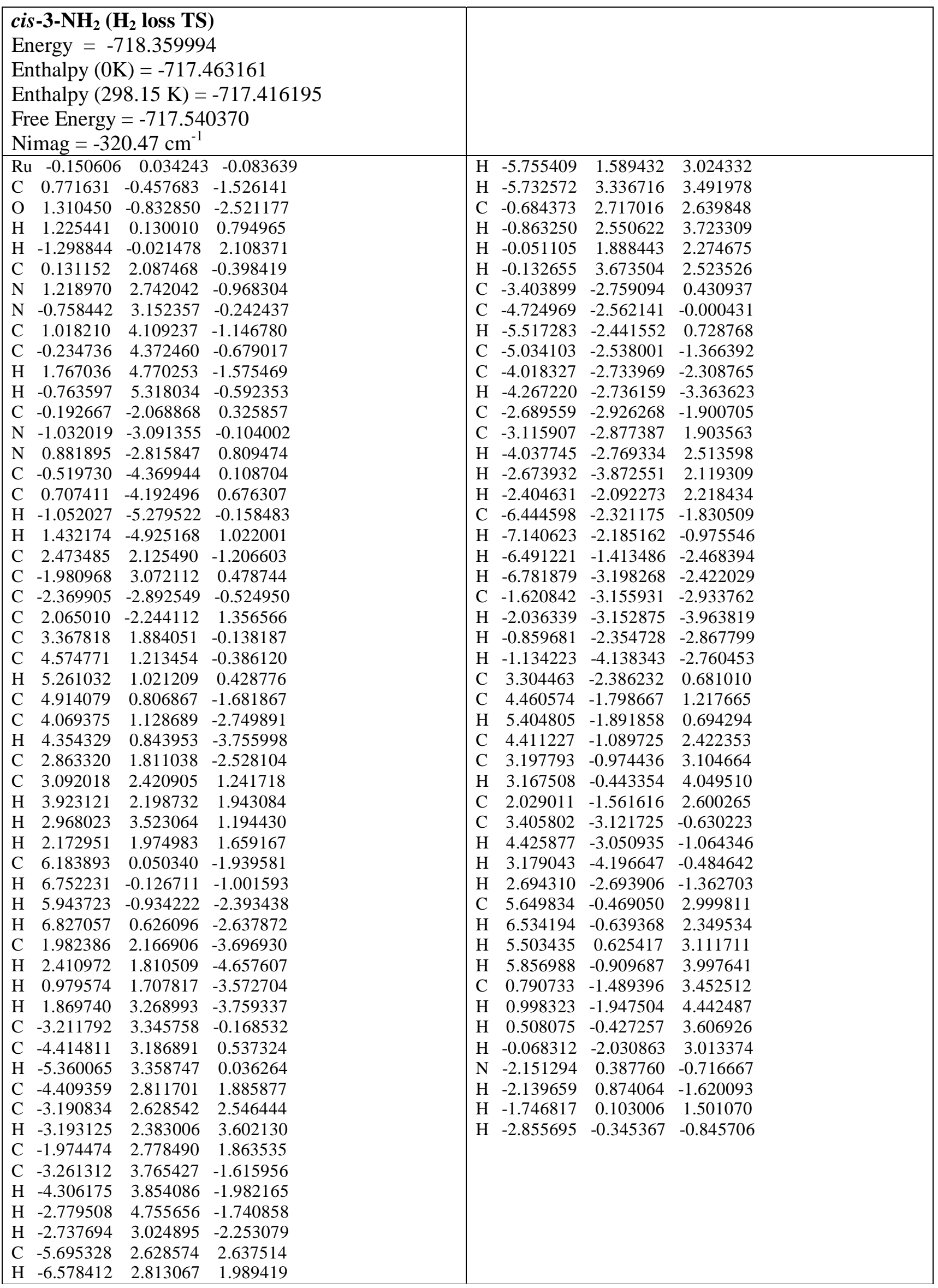




\begin{tabular}{|c|c|c|c|c|c|c|c|}
\hline \multicolumn{4}{|c|}{$\begin{array}{l}\mathbf{4 -} \mathbf{N H}_{\mathbf{2}}\left(\mathbf{T}_{\mathbf{H}}\right) \\
\text { Energy }=-717.224715 \\
\text { Enthalpy }(0 \mathrm{~K})=-716.341874 \\
\text { Enthalpy }(298.15 \mathrm{~K})=-716.295825 \\
\text { Free Energy }=-716.418913\end{array}$} & \multirow{2}{*}{\multicolumn{4}{|c|}{$\begin{array}{llll}\mathrm{H} & 5.924349 & 4.277881 & -2.470996\end{array}$}} \\
\hline \multirow{2}{*}{\multicolumn{4}{|c|}{$\begin{array}{llll}\mathrm{Ru} & 0.166225 & 0.008525 & -0.038165 \\
\mathrm{C} & 0.277354 & -0.956236 & 1.550502\end{array}$}} & & & & \\
\hline & & & & $\mathrm{C}$ & 0.956397 & 4.921143 & -1.127038 \\
\hline & 0.353507 & -1.593822 & 2.550363 & $\mathrm{H}$ & 1.108038 & 5.545221 & -2.033271 \\
\hline & -1.370921 & -0.220978 & 0.167377 & $\mathrm{H}$ & 0.017888 & 4.344151 & -1.262369 \\
\hline $\mathrm{C}$ & 0.077591 & 1.814262 & 0.986497 & $\mathrm{H}$ & 0.843432 & 5.609911 & -0.266414 \\
\hline & -0.868282 & 2.227314 & 1.913808 & $\mathrm{C}$ & 3.231402 & -2.142573 & -0.261088 \\
\hline & 0.946168 & 2.899525 & 0.983902 & $\mathrm{C}$ & 4.252134 & -2.275393 & 0.692232 \\
\hline & -0.618124 & 3.501870 & 2.427523 & $\mathrm{H}$ & 5.132401 & -1.646577 & 0.629776 \\
\hline & 0.532544 & 3.931474 & 1.835396 & $\mathrm{C}$ & 4.151081 & -3.217533 & 1.721193 \\
\hline & -1.257646 & 3.988668 & 3.159408 & $\mathrm{C}$ & 3.027914 & -4.047072 & 1.789650 \\
\hline $\mathrm{H}$ & 1.110091 & 4.837599 & 2.000187 & $\mathrm{H}$ & 2.957112 & -4.773780 & 2.590728 \\
\hline $\mathrm{C}$ & 0.202164 & $-1.721949-$ & -1.166050 & $\mathrm{C}$ & 1.995464 & -3.940718 & 0.844648 \\
\hline $\mathrm{N}$ & 1.038304 & -2.827023 & -1.135526 & $\mathrm{C}$ & 3.413721 & -1.176617 & -1.398942 \\
\hline $\mathrm{N}$ & -0.697790 & -2.075947 & -2.168730 & $\mathrm{H}$ & 4.409560 & -0.686806 & -1.368561 \\
\hline $\mathrm{C}$ & 0.625720 & -3.846978 & -2.002104 & $\mathrm{H}$ & 3.323745 & -1.712227 & -2.367280 \\
\hline C & -0.476943 & -3.375223 & -2.648364 & $\mathrm{H}$ & 2.646822 & -0.384813 & -1.341418 \\
\hline $\mathrm{H}$ & 1.176247 & -4.774712 & -2.136657 & $\mathrm{C}$ & 5.236030 & -3.350492 & 2.749495 \\
\hline $\mathrm{H}$ & -1.088012 & -3.832928 & -3.422482 & $\mathrm{H}$ & 6.061995 & -2.629965 & 2.568964 \\
\hline C & -2.118227 & 1.576203 & 2.075193 & $\mathrm{H}$ & 4.817378 & -3.157799 & 3.759718 \\
\hline $\mathrm{C}$ & 2.075855 & 2.998362 & 0.124470 & $\mathrm{H}$ & 5.654131 & -4.378716 & 2.719873 \\
\hline C & 2.084983 & -2.968588 & -0.183629 & $\mathrm{C}$ & 0.773928 & -4.809763 & 0.998587 \\
\hline C & -1.974897 & -1.463327 & -2.258561 & $\mathrm{H}$ & 0.828502 & -5.437177 & 1.913503 \\
\hline C & -3.086883 & 1.613153 & 1.042170 & & -0.132973 & -4.174626 & 1.073992 \\
\hline C & -4.315892 & 0.961779 & 1.223296 & $\mathrm{H}$ & 0.676526 & -5.494162 & 0.132570 \\
\hline $\mathrm{H}$ & -5.062288 & 0.993690 & 0.439340 & $\mathrm{C}$ & -2.993655 & -1.787944 & -1.326835 \\
\hline C & -4.591431 & 0.270192 & 2.408405 & C & -4.245674 & -1.162452 & -1.423590 \\
\hline C & -3.649252 & 0.268535 & 3.442669 & $\mathrm{H}$ & -5.023188 & -1.400553 & -0.707310 \\
\hline $\mathrm{H}$ & -3.877090 & -0.251259 & 4.366007 & $\mathrm{C}$ & -4.504667 & -0.233033 & -2.437207 \\
\hline C & -2.423059 & 0.934918 & 3.296463 & $\mathrm{C}$ & -3.515078 & 0.049185 & -3.383862 \\
\hline C & -2.840150 & 2.378845 & -0.232064 & $\mathrm{H}$ & -3.727383 & 0.748869 & -4.184399 \\
\hline $\mathrm{H}$ & -3.727863 & $2.364518-$ & -0.898681 & $\mathrm{C}$ & -2.260959 & -0.574071 & -3.320786 \\
\hline $\mathrm{H}$ & -2.605348 & 3.437683 & 0.005085 & $\mathrm{C}$ & -2.769717 & -2.816021 & -0.246960 \\
\hline $\mathrm{H}$ & -1.995297 & 1.931900 & -0.791634 & $\mathrm{H}$ & -3.675284 & -2.956694 & 0.380757 \\
\hline C & -5.890142 & -0.460445 & 2.584479 & $\mathrm{H}$ & -2.516197 & -3.794414 & -0.704933 \\
\hline $\mathrm{H}$ & -6.535488 & -0.370670 & 1.684636 & $\mathrm{H}$ & -1.944411 & -2.502819 & 0.421639 \\
\hline $\mathrm{H}$ & -5.689207 & -1.537578 & 2.764408 & $\mathrm{C}$ & -5.837311 & 0.450694 & -2.529394 \\
\hline $\mathrm{H}$ & -6.438937 & -0.042472 & 3.454505 & $\mathrm{H}$ & -6.520604 & 0.126952 & -1.715554 \\
\hline C & -1.436007 & 0.933353 & 4.433468 & $\mathrm{H}$ & -5.698528 & 1.549614 & -2.453350 \\
\hline $\mathrm{H}$ & -1.809568 & 0.349686 & 5.301490 & $\mathrm{H}$ & -6.312903 & 0.211577 & -3.503763 \\
\hline $\mathrm{H}$ & -0.476539 & 0.486241 & 4.102188 & $\mathrm{C}$ & -1.269785 & -0.281293 & -4.415676 \\
\hline $\mathrm{H}$ & -1.252228 & 1.973229 & 4.773692 & $\mathrm{H}$ & -1.707520 & -0.566302 & -5.395501 \\
\hline C & 3.185632 & 2.137198 & 0.299730 & $\mathrm{H}$ & -1.040618 & 0.805113 & -4.429428 \\
\hline C & 4.286097 & 2.234005 & -0.565413 & $\mathrm{H}$ & -0.319319 & -0.839203 & -4.295712 \\
\hline $\mathrm{H}$ & 5.138423 & - & -0.427912 & $\mathrm{~N}$ & 0.559314 & 1.044944 & -1.719296 \\
\hline C & 4.302471 & $3.175207-$ & -1.599769 & $\mathrm{H}$ & 0.286446 & 2.014222 & -1.898798 \\
\hline C & 3.221710 & $4.047718-$ & -1.755748 & $\mathrm{H}$ & 0.711982 & 0.591431 & -2.622468 \\
\hline $\mathrm{H}$ & 3.246344 & 4.780167 & -2.554348 & & & & \\
\hline C & 2.114282 & - & -0.895853 & & & & \\
\hline C & 3.249792 & 1.179282 & 1.456710 & & & & \\
\hline $\mathrm{H}$ & 4.231428 & 0.663191 & 1.511657 & & & & \\
\hline $\mathrm{H}$ & 3.094980 & 1.726895 & 2.410022 & & & & \\
\hline $\mathrm{H}$ & 2.472380 & 0.406188 & 1.343304 & & & & \\
\hline $\mathrm{C}$ & 5.471528 & 3.266151 & -2.536436 & & & & \\
\hline $\mathrm{H}$ & 6.252354 & 2.515067 & -2.291124 & & & & \\
\hline $\mathrm{H}$ & 5.129184 & 3.088303 & -3.577646 & & & & \\
\hline
\end{tabular}




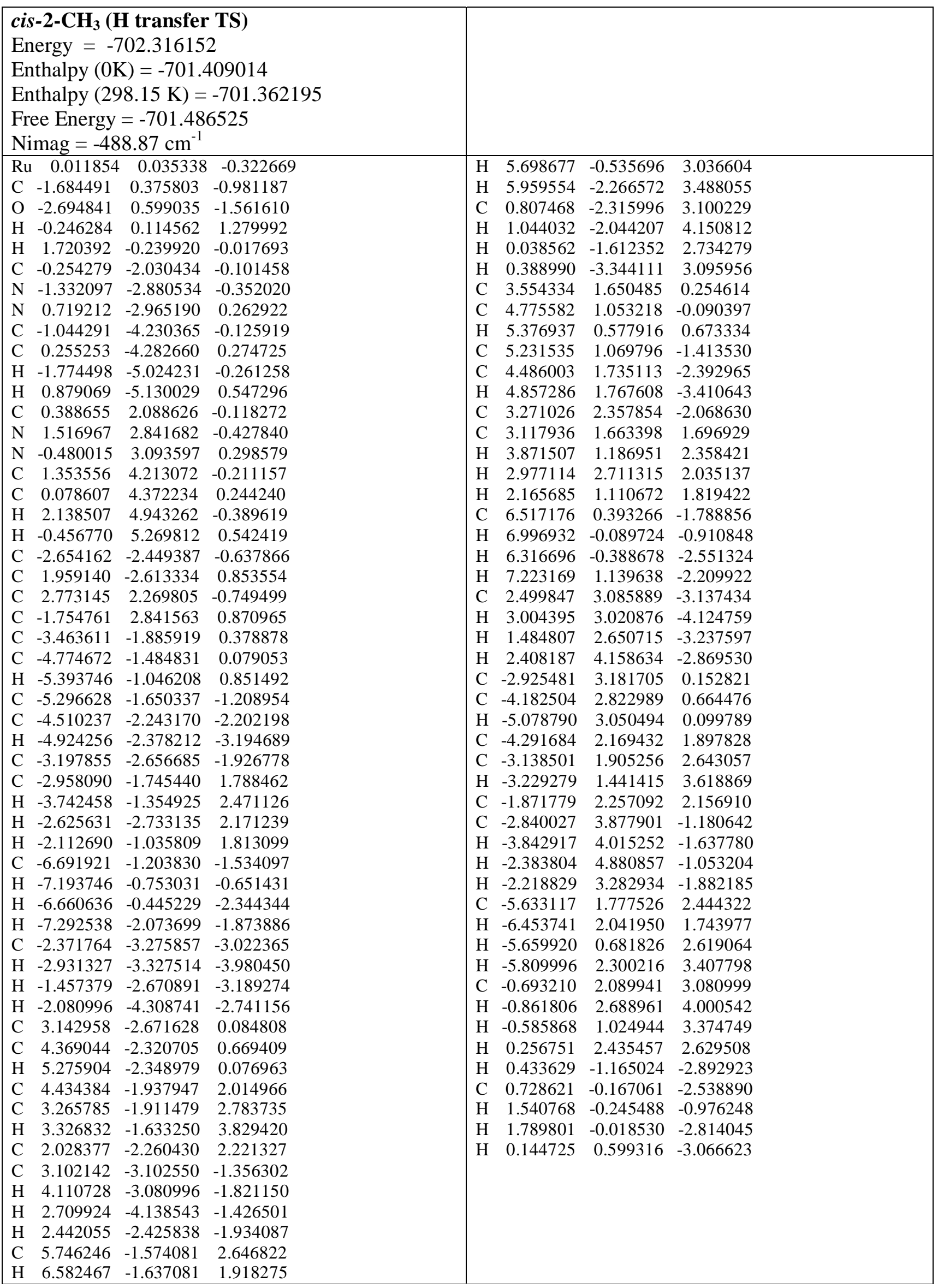




\begin{tabular}{|c|c|c|c|c|c|c|}
\hline \multicolumn{3}{|c|}{$\begin{array}{l}\text { cis-3-CH } \\
\text { Energy }=-702.321064 \\
\text { Enthalpy }(0 \mathrm{~K})=-701.411654 \\
\text { Enthalpy }(298.15 \mathrm{~K})=-701.364823 \\
\text { Free Energy }=-701.488577\end{array}$} & \multirow{2}{*}{\multicolumn{4}{|c|}{$\begin{array}{llll}\mathrm{H} & 6.070980 & -0.018897 & 2.524589\end{array}$}} \\
\hline & 0.00922 & $\begin{array}{lll}7 & 0.014790 & -0.089579\end{array}$ & & & & \\
\hline & -1.376590 & $0.191794-1.313095$ & $\mathrm{H}$ & 6.507179 & -1.691579 & 3.047754 \\
\hline & -2.212409 & $0.372262 \quad-2.127537$ & $\mathrm{C}$ & 1.383739 & -2.248766 & 2.992143 \\
\hline & -1.023710 & $-0.060001 \quad 1.189586$ & $\mathrm{H}$ & 1.669597 & -1.935267 & 4.018583 \\
\hline & 0.989192 & $0.092090 \quad 1.317630$ & $\mathrm{H}$ & 0.520836 & -1.632406 & 2.682746 \\
\hline & 0.061908 & $-2.090685 \quad-0.021964$ & $\mathrm{H}$ & 1.070410 & -3.313172 & 3.031753 \\
\hline & -0.921089 & $-3.008846-0.364929$ & $\mathrm{C}$ & 3.460883 & 2.117858 & 0.421152 \\
\hline & 1.149492 & $-2.942992 \quad 0.171591$ & $\mathrm{C}$ & 4.644790 & 1.652178 & -0.169585 \\
\hline & -0.474259 & $-4.331367-0.385664$ & $\mathrm{H}$ & 5.439109 & 1.260344 & 0.452358 \\
\hline & 0.843381 & $-4.290536-0.038968$ & $\mathrm{C}$ & 4.818419 & 1.704747 & -1.558207 \\
\hline & -1.115535 & $-5.173898 \quad-0.630861$ & $\mathrm{C}$ & 3.822905 & 2.278241 & -2.358315 \\
\hline & 1.562005 & $-5.095120 \quad 0.092652$ & $\mathrm{H}$ & 3.980117 & 2.354203 & -3.427876 \\
\hline & 0.076270 & $2.086198 \quad 0.145176$ & $\mathrm{C}$ & 2.629574 & 2.749253 & -1.788748 \\
\hline & 1.172700 & $2.949517 \quad 0.178651$ & $\mathrm{C}$ & 3.338547 & 2.150895 & 1.922497 \\
\hline & -0.958771 & $2.967025 \quad 0.431795$ & & 4.248108 & 1.750770 & 2.417532 \\
\hline & 0.813701 & $4.283067 \quad 0.413202$ & $\mathrm{H}$ & 3.196421 & 3.199687 & 2.257534 \\
\hline & -0.535654 & $4.288328 \quad 0.598995$ & & 2.472617 & 1.551500 & 2.262158 \\
\hline & 1.537618 & $5.093101 \quad 0.452218$ & $\mathrm{C}$ & 6.068521 & 1.172064 & -2.194254 \\
\hline & -1.212394 & $5.103162 \quad 0.843181$ & $\mathrm{H}$ & 6.765239 & 0.751697 & -1.437834 \\
\hline & -2.284537 & $-2.663502 \quad-0.545318$ & $\mathrm{H}$ & 5.805557 & 0.366853 & -2.912260 \\
\hline $\mathrm{C}$ & 2.407077 & $-2.518200 \quad 0.677144$ & $\mathrm{H}$ & 6.588773 & 1.988678 & -2.737643 \\
\hline & 2.417884 & $2.600042 \quad-0.398926$ & $\mathrm{C}$ & 1.596509 & 3.412211 & -2.663439 \\
\hline & -2.276583 & $2.548476 \quad 0.748286$ & $\mathrm{H}$ & 1.902471 & 3.412277 & -3.731079 \\
\hline $\mathrm{C}$ & -3.100103 & $-2.360412 \quad 0.569701$ & $\mathrm{H}$ & 0.622829 & 2.886906 & -2.582360 \\
\hline & -4.426016 & -1.950204 & & 1.460977 & 4.466749 & -2.345773 \\
\hline $\mathrm{H}$ & -5.050626 & $-1.712565 \quad 1.217103$ & & -3.323979 & 2.796110 & -0.168265 \\
\hline $\mathrm{C}$ & -4.960711 & $-1.874245 \quad-0.926055$ & & -4.623994 & 2.357374 & 0.126272 \\
\hline $\mathrm{C}$ & -4.175007 & $-2.247851-2.021705$ & & -5.423941 & 2.528389 & -0.584248 \\
\hline $\mathrm{H}$ & -4.600985 & $-2.207757-3.017466$ & $\mathrm{C}$ & -4.905610 & 1.725953 & 1.343754 \\
\hline $\mathrm{C}$ & -2.844573 & $-2.657191-1.843271$ & & -3.879166 & 1.530223 & 2.273693 \\
\hline $\mathrm{C}$ & -2.602722 & $-2.569825 \quad 1.975610$ & $\mathrm{H}$ & -4.105869 & 1.067724 & 3.227764 \\
\hline & -3.385508 & -2.336372 & & -2.570235 & 1.952624 & 2.000604 \\
\hline & -2.300226 & $\begin{array}{ll}-3.629706 & 2.108718\end{array}$ & $\mathrm{C}$ & -3.061046 & 3.526333 & -1.459809 \\
\hline $\mathrm{H}$ & -1.734203 & $-1.922644 \quad 2.183757$ & $\mathrm{H}$ & -3.975562 & 3.596563 & -2.086167 \\
\hline $\mathrm{C}$ & -6.372129 & $-1.415399-1.147366$ & $\mathrm{H}$ & -2.714539 & 4.557799 & -1.242860 \\
\hline $\mathrm{H}$ & -6.878537 & $-1.170528 \quad-0.190155$ & $\mathrm{H}$ & -2.278663 & 3.001839 & -2.045409 \\
\hline $\mathrm{H}$ & -6.371814 & $-0.507052-1.786076$ & $\mathrm{C}$ & -6.304511 & 1.300159 & 1.681887 \\
\hline $\mathrm{H}$ & -6.950371 & $-2.216588-1.653853$ & $\mathrm{H}$ & -7.008722 & 1.508328 & 0.848451 \\
\hline $\mathrm{C}$ & -2.014437 & $\begin{array}{ll}-3.027980 & -3.043201\end{array}$ & $\mathrm{H}$ & -6.326363 & 0.212538 & 1.896664 \\
\hline $\mathrm{H}$ & -2.590966 & $-2.931315 \quad-3.987596$ & $\mathrm{H}$ & -6.652461 & 1.849217 & 2.581983 \\
\hline $\mathrm{H}$ & -1.129758 & $-2.361498 \quad-3.109091$ & $\mathrm{C}$ & -1.541926 & 1.813508 & 3.095031 \\
\hline $\mathrm{H}$ & -1.673748 & $-4.080210 \quad-2.955976$ & $\mathrm{H}$ & -1.872302 & 2.389738 & 3.984729 \\
\hline C & 3.561749 & $-2.569261 \quad-0.141190$ & $\mathrm{H}$ & -1.440699 & 0.746328 & 3.384554 \\
\hline $\mathrm{C}$ & 4.778772 & $-2.066889 \quad 0.346225$ & $\mathrm{H}$ & -0.542801 & 2.191244 & 2.801345 \\
\hline $\mathrm{H}$ & 5.657077 & $-2.073202 \quad-0.288373$ & $\mathrm{H}$ & 0.982676 & -1.157869 & -2.449194 \\
\hline C & 4.879278 & $\begin{array}{ll}-1.579640 & 1.654678\end{array}$ & $\mathrm{C}$ & 1.257083 & -0.209490 & -1.958347 \\
\hline $\mathrm{C}$ & 3.758253 & $-1.608054 \quad 2.489618$ & $\mathrm{H}$ & 1.581600 & 0.062061 & 0.616131 \\
\hline $\mathrm{H}$ & 3.853676 & $-1.268214 \quad 3.514346$ & $\mathrm{H}$ & 2.341761 & -0.237076 & -1.755294 \\
\hline $\mathrm{C}$ & 2.527781 & $-2.094934 \quad 2.023208$ & $\mathrm{H}$ & 1.070351 & 0.584890 & -2.697958 \\
\hline C & 3.514361 & $-3.157003-1.527728$ & & & & \\
\hline $\mathrm{H}$ & 4.492833 & $-3.067623 \quad-2.045754$ & & & & \\
\hline $\mathrm{H}$ & 3.260275 & $-4.234844 \quad-1.471410$ & & & & \\
\hline $\mathrm{H}$ & 2.754867 & $-2.640598 \quad-2.143556$ & & & & \\
\hline $\mathrm{C}$ & 6.187126 & $\begin{array}{ll}-1.069227 & 2.185788\end{array}$ & & & & \\
\hline $\mathrm{H}$ & 6.983751 & $-1.102320 \quad 1.412357$ & & & & \\
\hline
\end{tabular}




\begin{tabular}{|c|c|c|c|c|c|c|c|}
\hline \multicolumn{4}{|c|}{$\begin{array}{l}\text { cis-3-CH } \mathbf{C}\left(\mathbf{H}_{\mathbf{2}} \text { loss } \mathbf{T S}\right) \\
\text { Energy }=-702.293277 \\
\text { Enthalpy }(0 \mathrm{~K})=-701.386470 \\
\text { Enthalpy }(298.15 \mathrm{~K})=-701.339589 \\
\text { Free Energy }=-701.463531 \\
\text { Nimag }=-223.37 \mathrm{~cm}^{-1}\end{array}$} & \multirow{2}{*}{\multicolumn{4}{|c|}{$\begin{array}{llll}\mathrm{H} & -6.035748 & 0.351890 & 1.883167\end{array}$}} \\
\hline & -0.02090 & 0.096458 & -0.043810 & & & & \\
\hline $\mathrm{C}$ & 1.092335 & -0.109129 & -1.408094 & & -6.392866 & 1.896124 & 2.751372 \\
\hline & 1.796916 & -0.259844 & -2.356924 & & -1.277131 & 2.241345 & 3.004421 \\
\hline & 1.210324 & -0.048358 & 1.076904 & & -1.607171 & 1.728464 & 3.932737 \\
\hline & -0.439055 & -0.479753 & 2.248319 & & -0.363174 & 1.742184 & 2.638020 \\
\hline & 0.159198 & 2.198938 & 0.073152 & & -1.016282 & 3.289162 & 3.262666 \\
\hline $\mathrm{N}$ & 1.251392 & 3.000250 & -0.228751 & & -3.801638 & -2.149560 & 0.362554 \\
\hline & -0.819260 & 3.176889 & 0.287035 & & -5.002630 & -1.960587 & -0.337797 \\
\hline $\mathrm{C}$ & 0.967315 & 4.364544 & -0.229118 & & -5.900268 & -1.676802 & 0.198457 \\
\hline & -0.347914 & 4.479307 & 0.111451 & & -5.055614 & -2.137764 & -1.724924 \\
\hline & 1.710242 & 5.128461 & -0.443077 & & -3.905197 & -2.532807 & -2.414911 \\
\hline $\mathrm{H}$ & -0.946675 & 5.367609 & 0.292737 & & -3.959871 & -2.693626 & -3.485359 \\
\hline & -0.247750 & -1.978684 & 0.207611 & & -2.691431 & -2.725612 & -1.736968 \\
\hline & -1.418954 & -2.730037 & 0.370973 & & -3.784061 & -1.974103 & 1.855682 \\
\hline $\mathrm{N}$ & 0.704013 & -2.878233 & 0.671837 & & -4.793821 & -1.747398 & 2.257172 \\
\hline & -1.174284 & -4.005615 & 0.896564 & & -3.424491 & -2.902817 & 2.344854 \\
\hline C & 0.170697 & -4.091282 & 1.099008 & & -3.116067 & -1.133503 & 2.118675 \\
\hline $\mathrm{H}$ & -1.961913 & -4.731503 & 1.084908 & & -6.335810 & -1.922913 & -2.477836 \\
\hline $\mathrm{H}$ & 0.776428 & -4.896978 & 1.505903 & & -7.162039 & -1.616187 & -1.801635 \\
\hline C & 2.546535 & 2.491915 & -0.499392 & & -6.191298 & -1.126625 & -3.238105 \\
\hline & -2.142811 & 2.880586 & 0.710306 & & -6.630724 & -2.863567 & -2.988835 \\
\hline C & -2.622073 & -2.482847 & -0.342689 & & -1.487943 & -3.202449 & -2.506473 \\
\hline C & 2.086846 & -2.577188 & 0.778485 & $\mathrm{H}$ & -1.729748 & -3.389509 & -3.574197 \\
\hline $\mathrm{C}$ & 3.368739 & 2.036812 & 0.556581 & $\mathrm{H}$ & -0.690470 & -2.434703 & -2.472039 \\
\hline C & 4.633889 & 1.503353 & 0.266586 & & -1.109241 & -4.149636 & -2.068696 \\
\hline $\mathrm{H}$ & 5.267467 & 1.157889 & 1.073305 & $\mathrm{C}$ & 2.938985 & -2.809517 & -0.325429 \\
\hline C & 5.102166 & 1.448761 & -1.052609 & C & 4.311426 & -2.535783 & -0.215640 \\
\hline C & 4.308801 & 1.951884 & -2.088774 & $\mathrm{H}$ & 4.964480 & -2.707482 & -1.063024 \\
\hline $\mathrm{H}$ & 4.683985 & 1.923429 & -3.105192 & $\mathrm{C}$ & 4.857419 & -2.102961 & 0.998760 \\
\hline C & 3.042497 & 2.493514 & -1.823499 & C & 4.021880 & -1.920304 & 2.104610 \\
\hline C & 2.956297 & 2.221233 & 1.994221 & $\mathrm{H}$ & 4.450014 & -1.603227 & 3.048964 \\
\hline $\mathrm{H}$ & 3.729982 & 1.842520 & 2.695511 & $\mathrm{C}$ & 2.646195 & -2.174478 & 2.016699 \\
\hline $\mathrm{H}$ & 2.802793 & 3.301569 & 2.198881 & $\mathrm{C}$ & 2.408818 & -3.438384 & -1.587050 \\
\hline $\mathrm{H}$ & 2.014616 & 1.684852 & 2.208488 & $\mathrm{H}$ & 3.205683 & -3.567286 & -2.349901 \\
\hline C & 6.456800 & 0.885472 & -1.367108 & $\mathrm{H}$ & 1.987115 & -4.438739 & -1.354697 \\
\hline $\mathrm{H}$ & 6.991750 & 0.566338 & -0.449200 & $\mathrm{H}$ & 1.611224 & -2.811969 & -2.026006 \\
\hline $\mathrm{H}$ & 6.348157 & 0.006646 & -2.037067 & $\mathrm{C}$ & 6.336095 & -1.893796 & 1.146391 \\
\hline $\mathrm{H}$ & 7.072967 & 1.656857 & -1.875239 & $\mathrm{H}$ & 6.870954 & -2.059494 & 0.187078 \\
\hline C & 2.208961 & 3.023433 & -2.960941 & $\mathrm{H}$ & 6.538806 & -0.859628 & 1.491397 \\
\hline $\mathrm{H}$ & 2.720721 & 2.897664 & -3.938644 & $\mathrm{H}$ & 6.737256 & -2.604007 & 1.899627 \\
\hline $\mathrm{H}$ & 1.240614 & 2.482169 & -3.005143 & & 1.822049 & -2.019873 & 3.267120 \\
\hline $\mathrm{H}$ & 2.014692 & 4.105717 & -2.814889 & $\mathrm{H}$ & 2.230269 & -2.678174 & 4.062685 \\
\hline C & -3.255928 & 3.199211 & -0.108822 & $\mathrm{H}$ & 1.867433 & -0.965789 & 3.613802 \\
\hline C & -4.527223 & 2.715341 & 0.240313 & $\mathrm{H}$ & 0.760450 & -2.295444 & 3.120661 \\
\hline $\mathrm{H}$ & -5.375691 & 2.917665 & -0.402502 & $\mathrm{H}$ & -1.726412 & 1.414768 & -1.808854 \\
\hline C & -4.715417 & 1.977129 & 1.415174 & & -1.772338 & 0.420566 & -1.335648 \\
\hline $\mathrm{C}$ & -3.634655 & 1.765287 & 2.277379 & $\mathrm{H}$ & -1.032655 & -0.532460 & 2.731062 \\
\hline $\mathrm{H}$ & -3.800653 & 1.259102 & 3.221257 & $\mathrm{H}$ & -2.740734 & 0.364441 & -0.788207 \\
\hline C & -2.356924 & 2.244507 & 1.953378 & & -1.839783 & -0.281853 & -2.183403 \\
\hline C & -3.106974 & 4.019366 & -1.365946 & & & & \\
\hline $\mathrm{H}$ & -4.061577 & 4.081768 & -1.930316 & & & & \\
\hline $\mathrm{H}$ & -2.808063 & 5.054482 & -1.106320 & & & & \\
\hline $\mathrm{H}$ & -2.342661 & 3.580589 & -2.037271 & & & & \\
\hline C & -6.073608 & 1.455810 & 1.783390 & & & & \\
\hline & -6.832689 & 1.710610 & 1.013556 & & & & \\
\hline
\end{tabular}




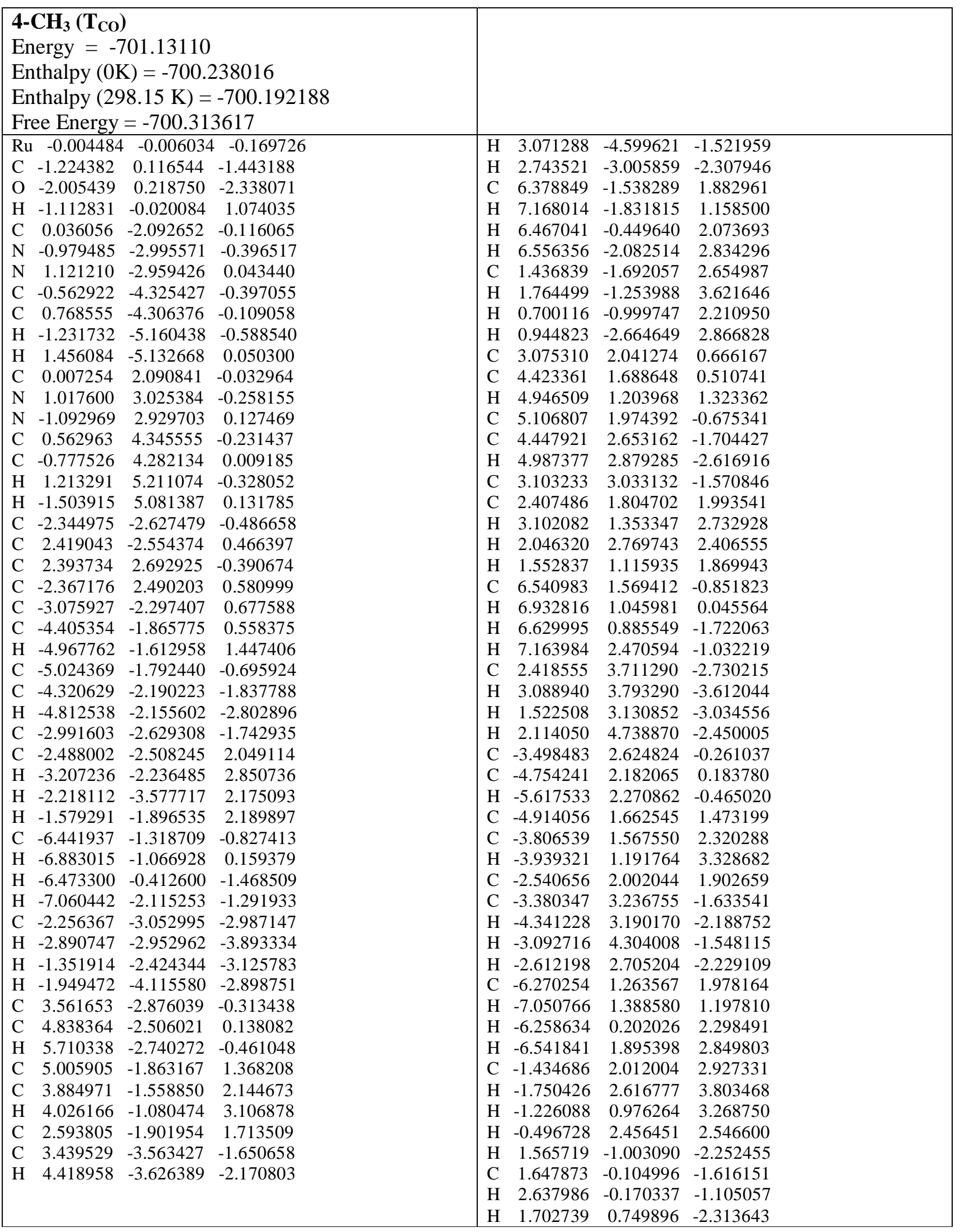




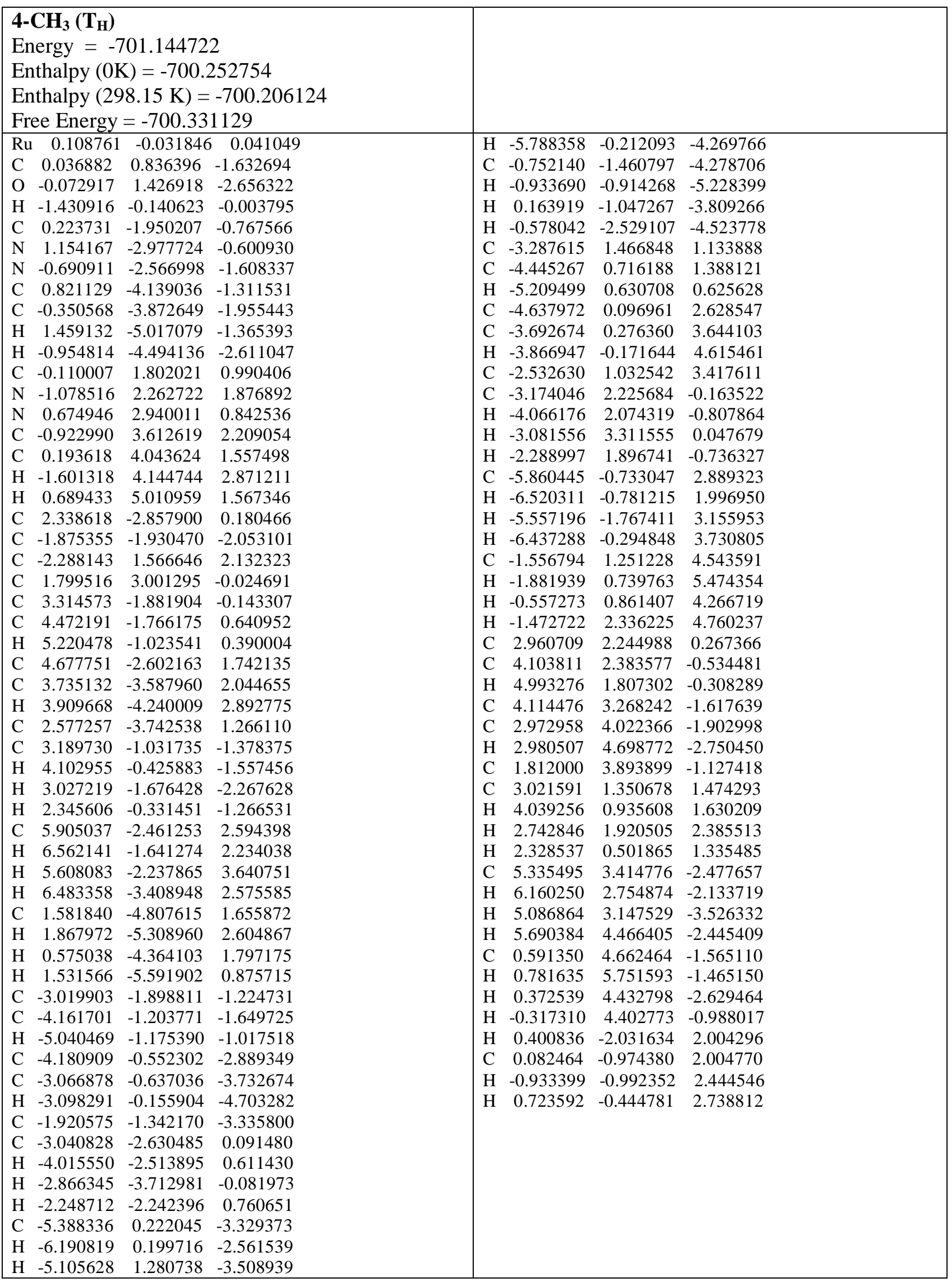




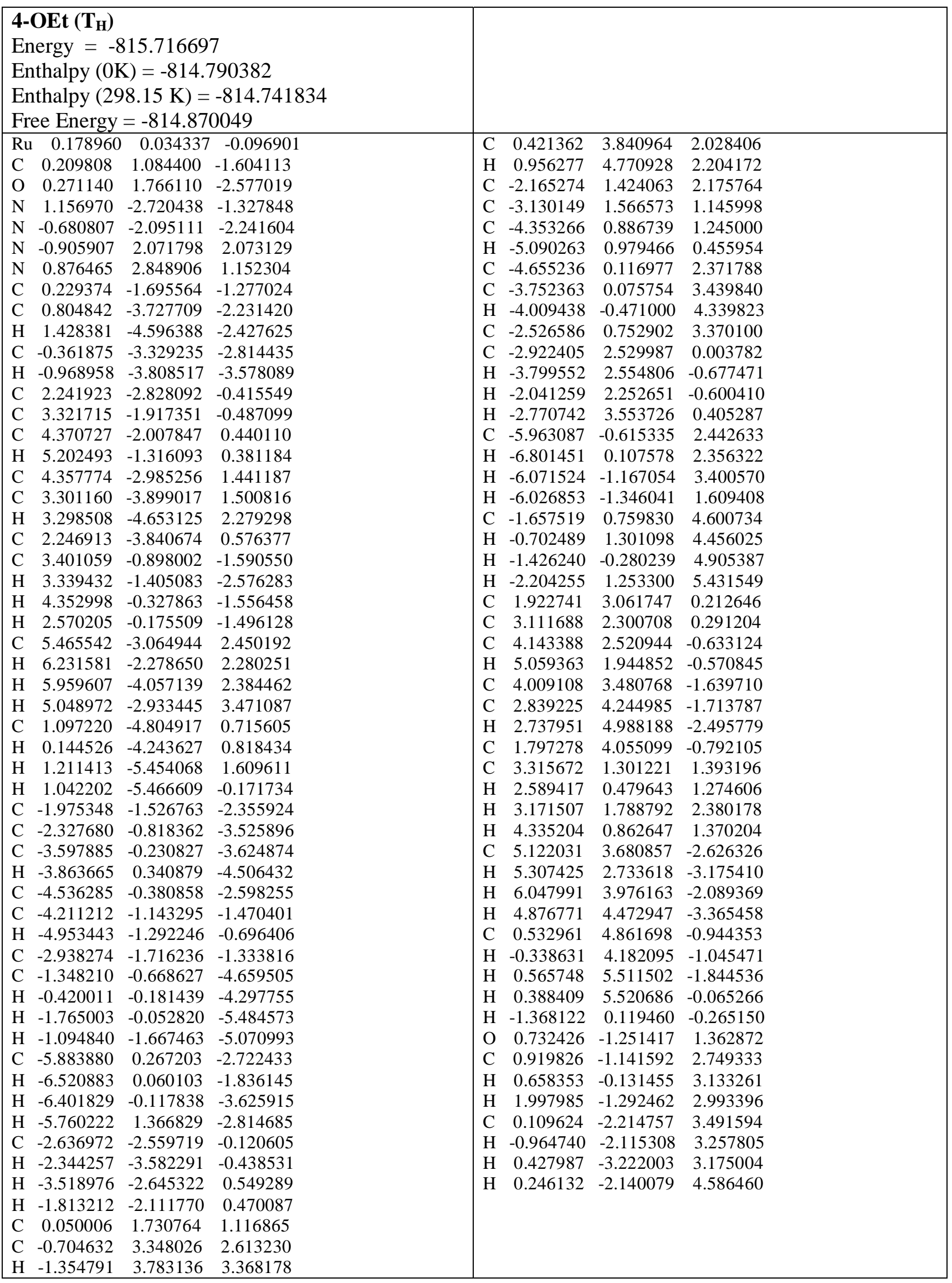




\begin{tabular}{|c|c|c|c|c|c|}
\hline \multicolumn{3}{|c|}{$\begin{array}{l}\mathbf{4 - S i H} \mathbf{H}_{\mathbf{3}}\left(\mathbf{T}_{\mathbf{H}}\right) \\
\text { Energy }=-666.962085 \\
\text { Enthalpy }(0 \mathrm{~K})=-666.080526 \\
\text { Enthalpy }(298.15 \mathrm{~K})=-666.033425 \\
\text { Free Energy }=-666.157440\end{array}$} & \multirow{2}{*}{\multicolumn{3}{|c|}{$\begin{array}{llll}\text { C } & 0.038279 & 4.261075 & -1.027774\end{array}$}} \\
\hline & -0.04759 & $\begin{array}{lll} & 0.031646 & -0.013098\end{array}$ & & & \\
\hline $\mathrm{C}$ & 0.069479 & 0.7115891 .749903 & H -0.471561 & 5.220157 & -0.990358 \\
\hline $\mathrm{O}$ & 0.253679 & $1.156550 \quad 2.830925$ & C 2.507677 & 1.816085 & -1.845902 \\
\hline $\mathrm{N}$ & -1.271985 & $-2.926343 \quad 0.236448$ & 3.456949 & 1.345740 & -0.908375 \\
\hline $\mathrm{N}$ & 0.661771 & $-2.765569 \quad 1.163190$ & 4.501373 & 0.514845 & -1.339387 \\
\hline $\mathrm{N}$ & 1.368181 & $2.541221-1.410224$ & H $\quad 5.218315$ & 0.129573 & -0.623943 \\
\hline $\mathrm{N}$ & -0.502171 & $3.078882-0.512313$ & C 4.648504 & 0.196583 & -2.693235 \\
\hline & -0.287906 & $-1.979765 \quad 0.523196$ & 3.774822 & 0.756536 & -3.633937 \\
\hline $\mathrm{C}$ & -0.948702 & $-4.203893 \quad 0.713562$ & H 3.916860 & 0.544543 & -4.686938 \\
\hline & -1.618402 & $-5.057208 \quad 0.657007$ & C 2.722657 & 1.591583 & -3.226097 \\
\hline $\mathrm{C}$ & 0.265020 & $-4.093884 \quad 1.320557$ & 3.434268 & 1.818677 & 0.522140 \\
\hline $\mathrm{H}$ & 0.873890 & $-4.841171 \quad 1.822565$ & H 4.283977 & 1.405313 & 1.105754 \\
\hline $\mathrm{C}$ & -2.489792 & $-2.632124 \quad-0.444863$ & H 2.500624 & 1.515836 & 1.025247 \\
\hline $\mathrm{C}$ & -3.384315 & $-1.662359 \quad 0.076384$ & H 3.507464 & 2.926161 & 0.546040 \\
\hline $\mathrm{C}$ & -4.577562 & $-1.375659-0.603481$ & 5.761620 & -0.712798 & -3.123797 \\
\hline $\mathrm{H}$ & -5.266056 & $-0.644086 \quad-0.198955$ & H 6.738034 & -0.272986 & -2.830370 \\
\hline $\mathrm{C}$ & -4.898410 & $-2.029999-1.795256$ & H 5.761646 & -0.867294 & -4.223768 \\
\hline $\mathrm{C}$ & -4.039667 & $-3.011169 \quad-2.294647$ & 5.646026 & -1.701071 & -2.630956 \\
\hline $\mathrm{H}$ & -4.304927 & $-3.523151 \quad-3.212525$ & 1.821723 & 2.208504 & -4.265867 \\
\hline $\mathrm{C}$ & -2.850843 & $-3.340334-1.624270$ & H $\quad 0.761395$ & 1.952403 & -4.062166 \\
\hline $\mathrm{C}$ & -3.141512 & $-1.011960 \quad 1.410846$ & H 2.073759 & 1.856037 & -5.288521 \\
\hline $\mathrm{H}$ & -2.954641 & $-1.787797 \quad 2.182766$ & H 1.933378 & 3.311642 & -4.252193 \\
\hline $\mathrm{H}$ & -4.011858 & $-0.406847 \quad 1.741557$ & C -1.685975 & 3.033058 & 0.273663 \\
\hline $\mathrm{H}$ & -2.275044 & $-0.334272 \quad 1.341938$ & C -2.811464 & 2.304615 & -0.180004 \\
\hline $\mathrm{C}$ & -6.161469 & $-1.700720 \quad-2.536019$ & C -3.982737 & 2.282704 & 0.591086 \\
\hline $\mathrm{H}$ & -6.743371 & $-0.906792 \quad-2.021144$ & H -4.853615 & 1.743197 & 0.239290 \\
\hline $\mathrm{H}$ & -6.796651 & $-2.608229-2.612808$ & C -4.046085 & 2.949888 & 1.816874 \\
\hline $\mathrm{H}$ & -5.911679 & $-1.344769-3.557708$ & C -2.937116 & 3.674533 & 2.266952 \\
\hline C & -1.953597 & $-4.389452 \quad-2.232447$ & H -2.990105 & 4.192208 & 3.217389 \\
\hline $\mathrm{H}$ & -0.930802 & $\begin{array}{ll}-3.988125 & -2.370509\end{array}$ & C -1.761559 & 3.737894 & 1.502149 \\
\hline $\mathrm{H}$ & -2.322967 & $-4.719841 \quad-3.226537$ & C -2.802544 & 1.615239 & -1.516581 \\
\hline $\mathrm{H}$ & -1.915063 & $\begin{array}{ll}-5.288170 & -1.587449\end{array}$ & H -2.071796 & 0.787333 & -1.500580 \\
\hline $\mathrm{C}$ & 1.746452 & $-2.206846 \quad 1.884644$ & H -2.530214 & 2.338321 & -2.313859 \\
\hline $\mathrm{C}$ & 1.568817 & $-1.784614 \quad 3.222096$ & H -3.795143 & 1.185391 & -1.767273 \\
\hline $\mathrm{C}$ & 2.631404 & $-1.166050 \quad 3.898270$ & C -5.303544 & 2.881978 & 2.633011 \\
\hline $\mathrm{H}$ & 2.495495 & $-0.813447 \quad 4.914034$ & H $\quad-5.526227$ & 1.823795 & 2.885757 \\
\hline $\mathrm{C}$ & 3.875039 & $-1.002841 \quad 3.277672$ & H -6.150083 & 3.301461 & 2.049638 \\
\hline $\mathrm{C}$ & 4.070411 & $-1.491889 \quad 1.980081$ & H $\quad-5.209566$ & 3.456710 & 3.578888 \\
\hline $\mathrm{H}$ & 5.042589 & $-1.392626 \quad 1.512348$ & C -0.577361 & 4.495108 & 2.047297 \\
\hline $\mathrm{C}$ & 3.019782 & $-2.104799 \quad 1.281232$ & H $\quad 0.309585$ & 3.829491 & 2.095469 \\
\hline $\mathrm{C}$ & 0.259078 & $-2.001623 \quad 3.935020$ & H -0.771926 & 4.880930 & 3.070528 \\
\hline $\mathrm{H}$ & -0.566017 & $-1.502891 \quad 3.387443$ & H -0.349036 & 5.367284 & 1.403283 \\
\hline $\mathrm{H}$ & 0.282047 & $-1.595364 \quad 4.968426$ & H 1.472448 & -0.241245 & -0.028724 \\
\hline $\mathrm{H}$ & 0.043569 & $-3.088393 \quad 3.998039$ & $\mathrm{Si} \quad 0.129336$ & -0.989618 & -2.282678 \\
\hline $\mathrm{C}$ & 4.988537 & $-0.319091 \quad 4.015114$ & H $\quad 0.786575$ & -2.358641 & -2.357948 \\
\hline $\mathrm{H}$ & 5.912911 & $-0.264644 \quad 3.401499$ & H $\quad 0.764557$ & -0.307756 & -3.479621 \\
\hline $\mathrm{H}$ & 5.217271 & $-0.877245 \quad 4.947320$ & H $\quad-1.268904$ & -1.285593 & -2.848068 \\
\hline $\mathrm{H}$ & 4.680817 & $0.715225 \quad 4.276823$ & & & \\
\hline $\mathrm{C}$ & 3.268894 & $\begin{array}{ll}-2.662261 & -0.094229\end{array}$ & & & \\
\hline $\mathrm{H}$ & 3.035695 & $\begin{array}{ll}-3.747510 & -0.106237\end{array}$ & & & \\
\hline $\mathrm{H}$ & 4.327059 & $-2.532022-0.405250$ & & & \\
\hline $\mathrm{H}$ & 2.631362 & $-2.144917 \quad-0.835476$ & & & \\
\hline $\mathrm{C}$ & 0.306886 & $1.964483-0.724791$ & & & \\
\hline $\mathrm{C}$ & 1.219683 & $3.914340-1.611664$ & & & \\
\hline $\mathrm{H}$ & 1.960436 & $4.528024 \quad-2.117220$ & & & \\
\hline
\end{tabular}




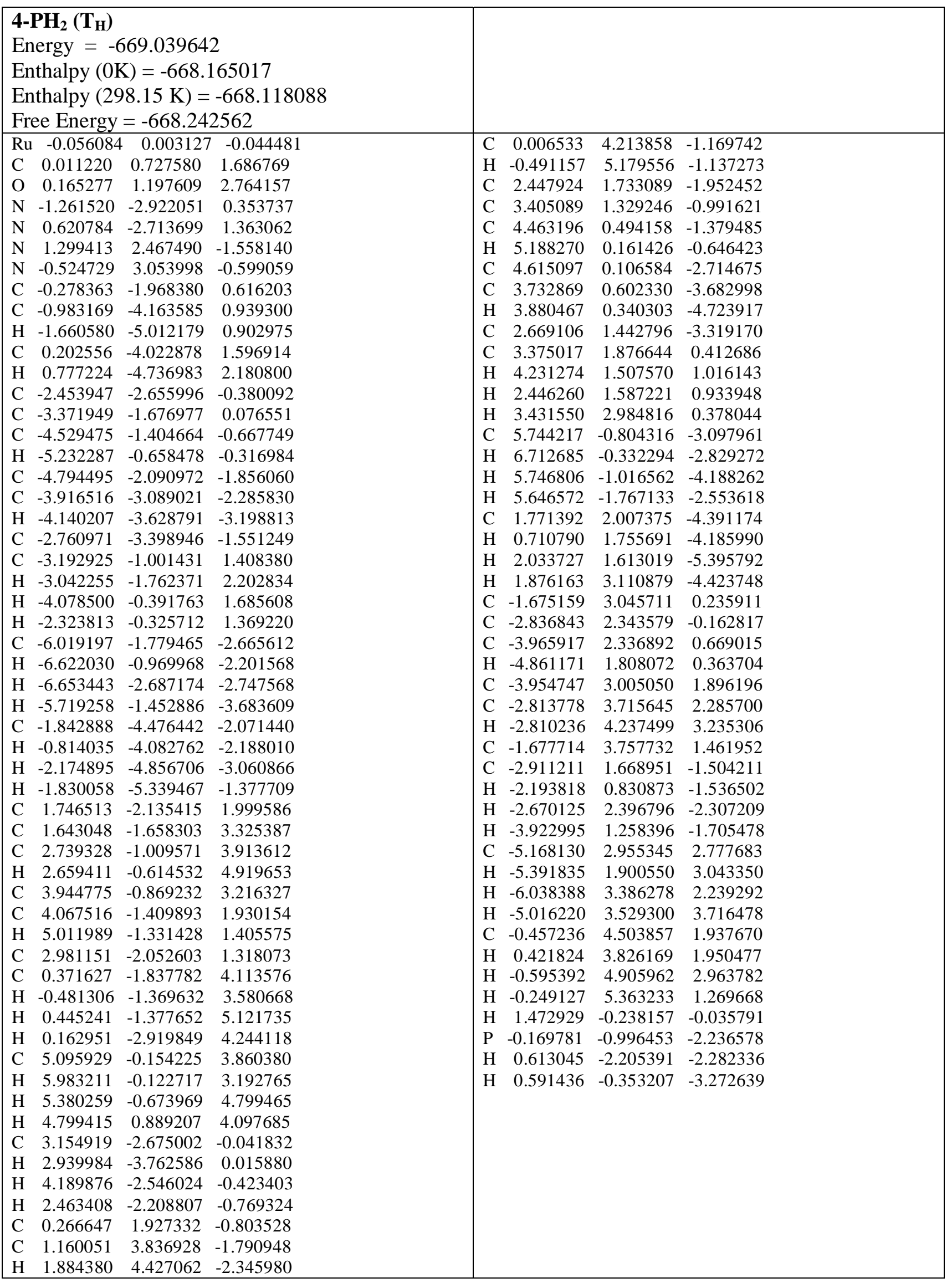




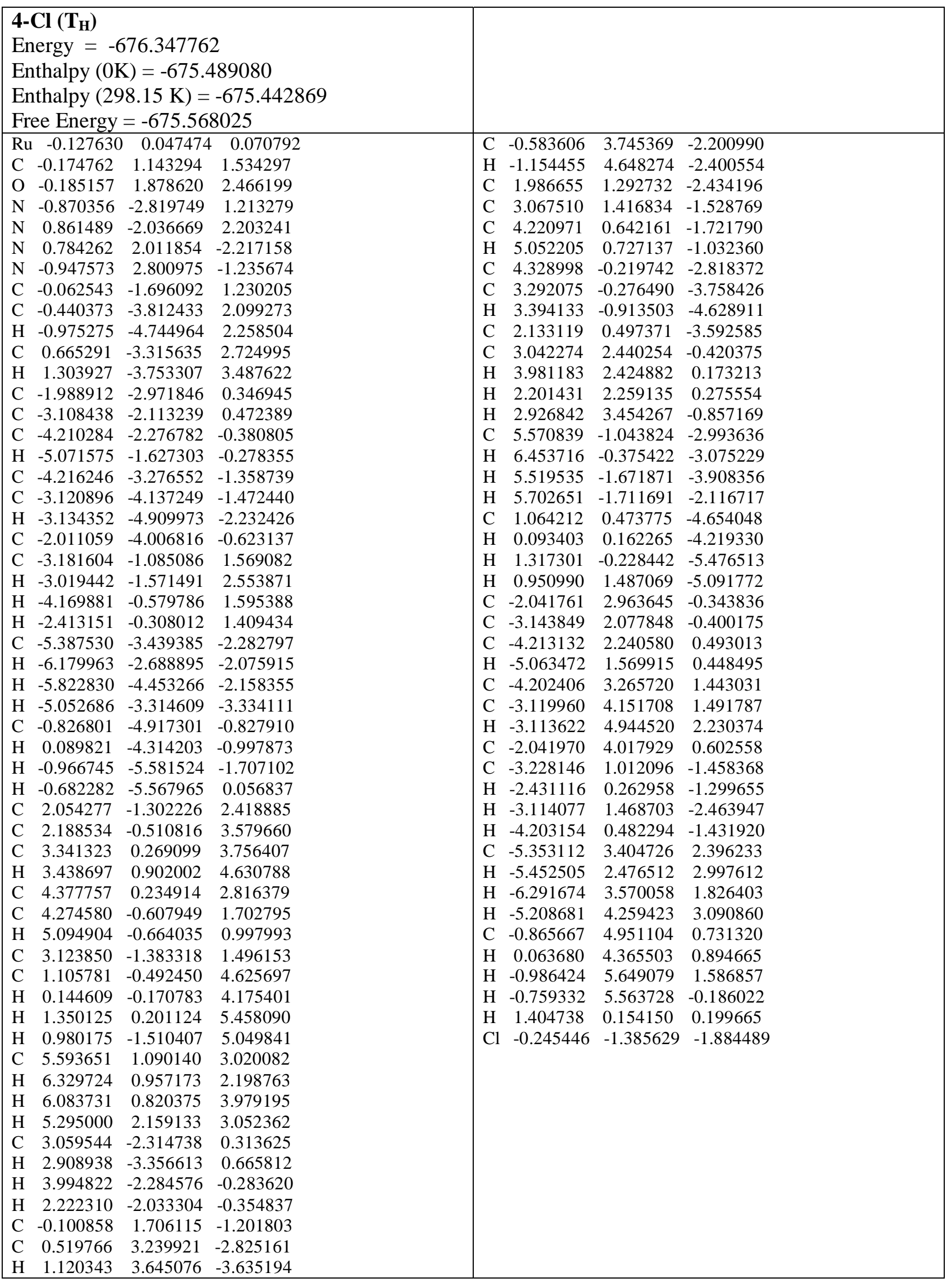




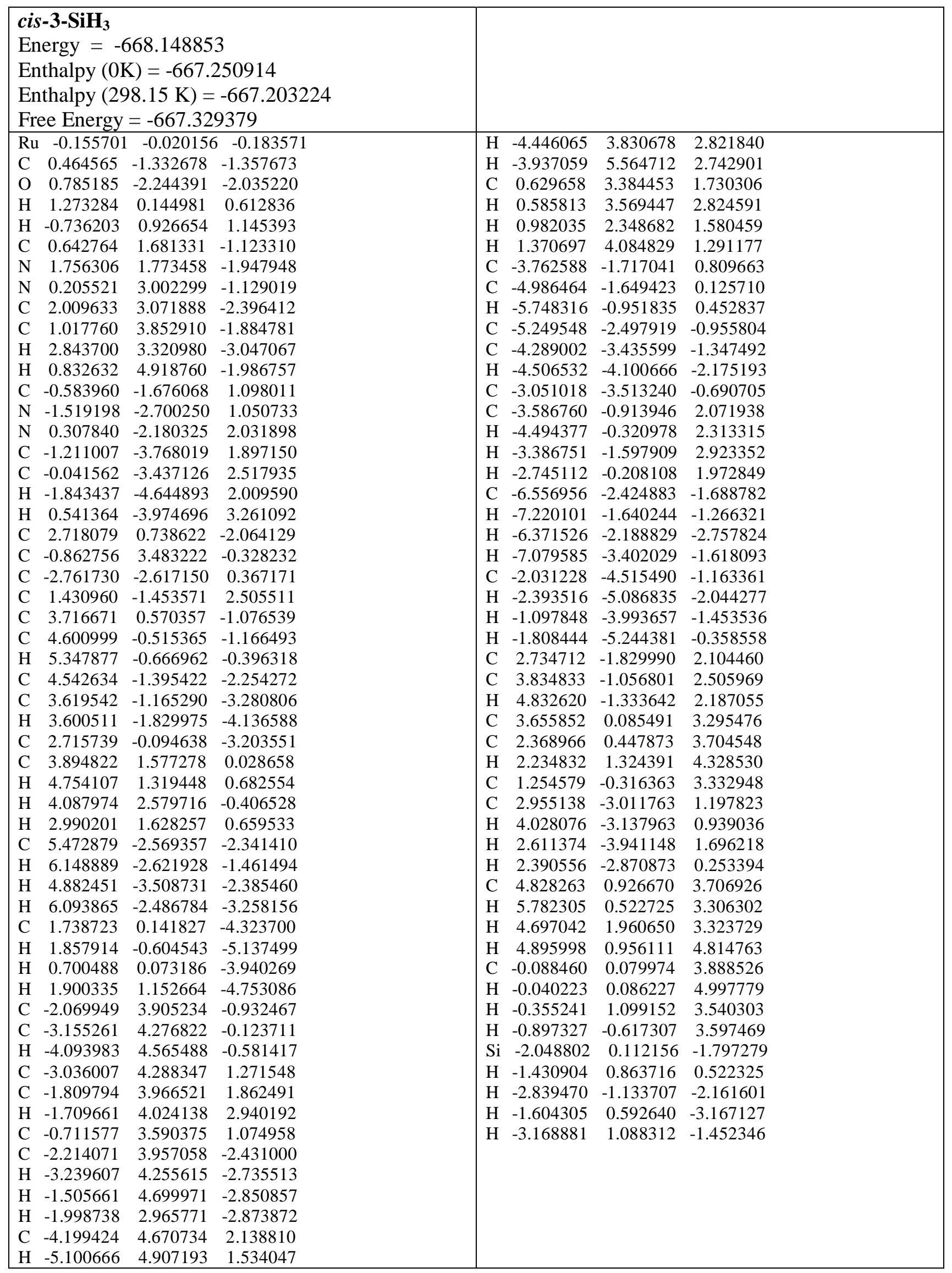




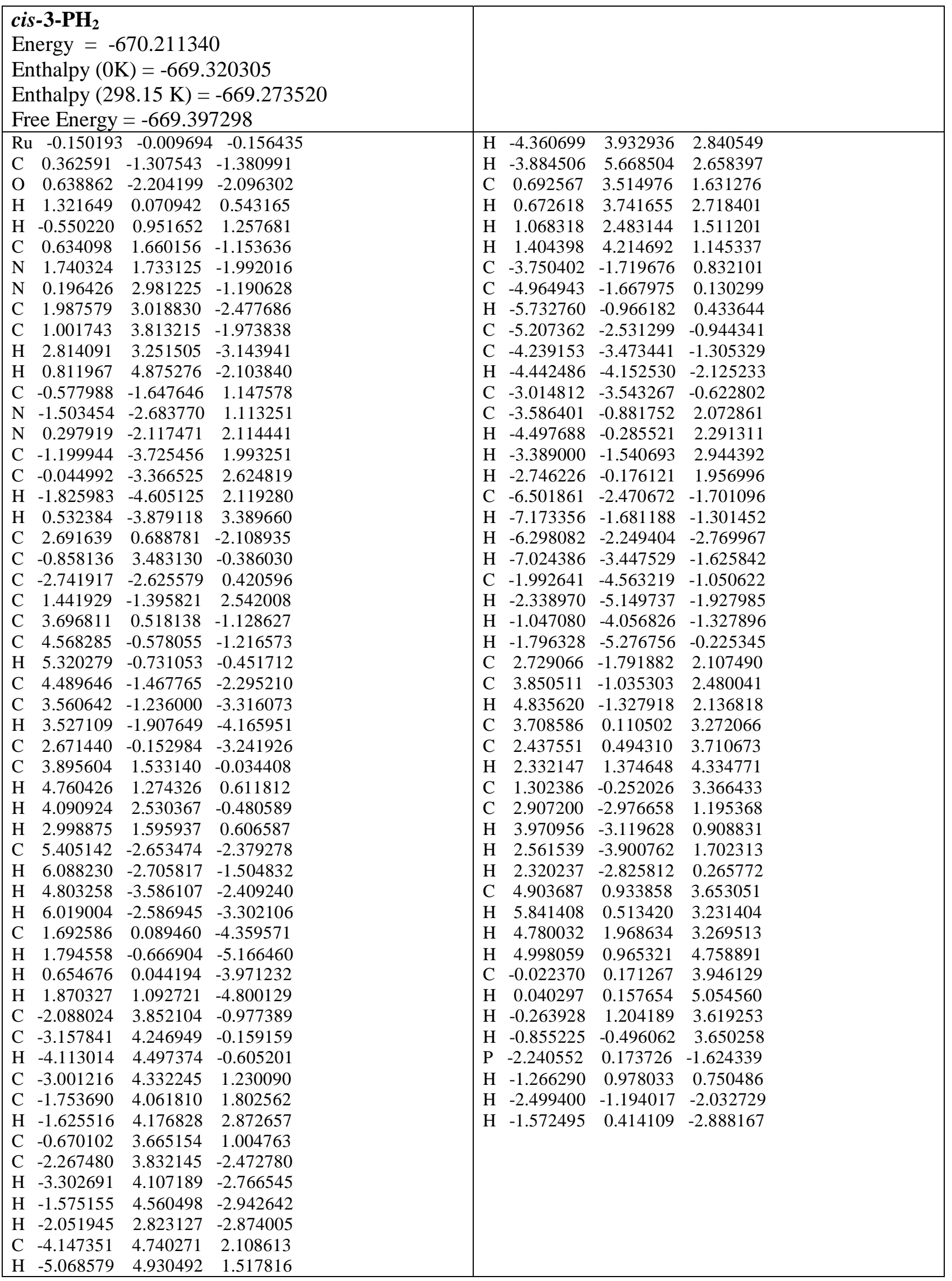




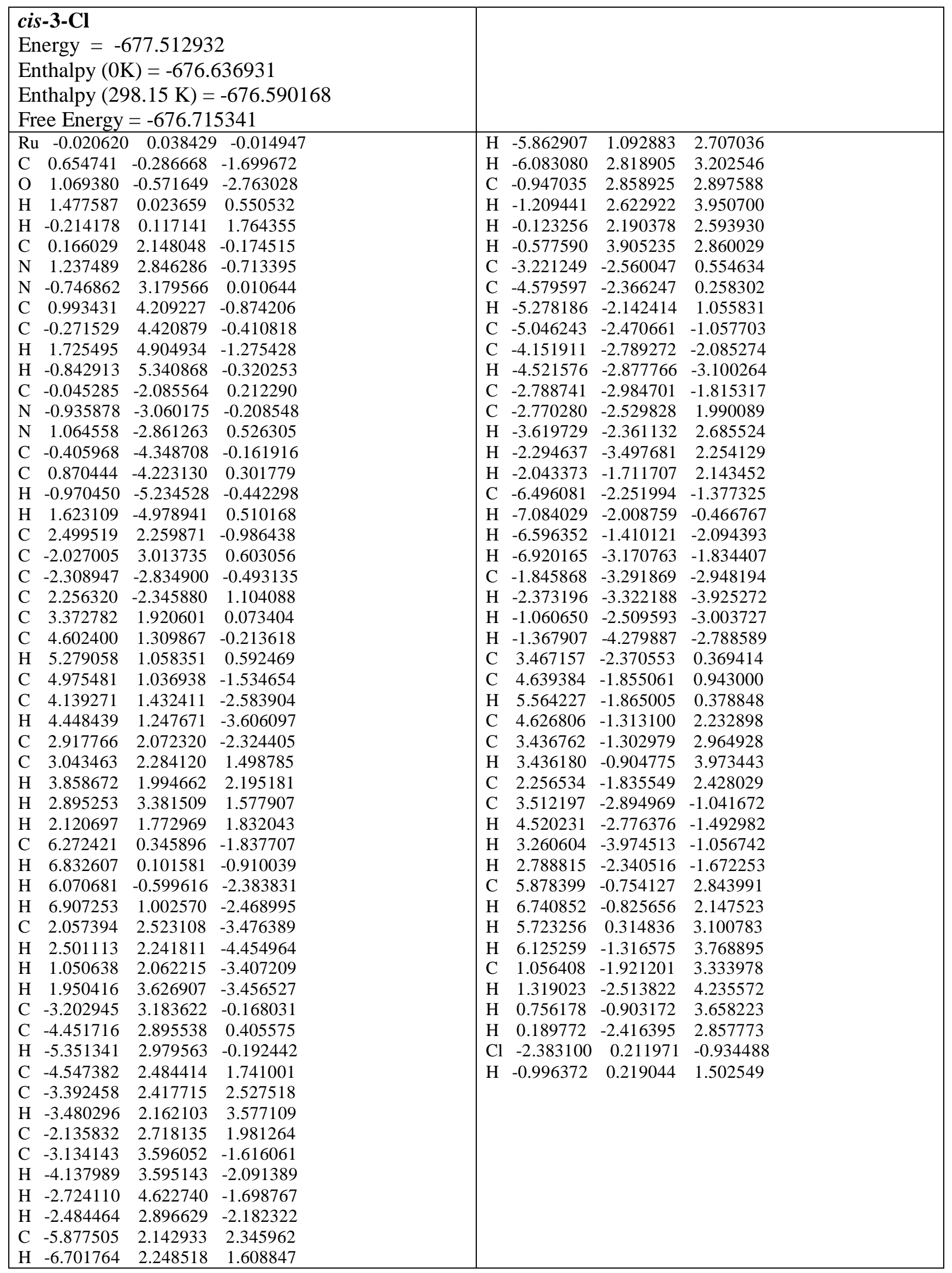




\begin{tabular}{|c|c|}
\hline $\begin{array}{l}\mathbf{H}_{2} \\
\text { Energy }=-1.176465 \\
\text { Enthalpy }(0 \mathrm{~K})=-1.166543 \\
\text { Enthalpy }(298.15 \mathrm{~K})=-1.163238 \\
\text { Free Energy }=-1.178050\end{array}$ & $\begin{array}{l}\mathbf{S H}_{2} \\
\text { Energy }=-11.424456 \\
\text { Enthalpy }(0 \mathrm{~K})=-11.409820 \\
\text { Enthalpy }(298.15 \mathrm{~K})=-11.406026 \\
\text { Free Energy }=-11.429413\end{array}$ \\
\hline $\begin{array}{cccc}\mathrm{H} & 0.000000 & 0.000000 & 0.375288 \\
\mathrm{H} & 0.000000 & 0.000000 & -0.375288\end{array}$ & $\begin{array}{cccc}\mathrm{S} & 0.000000 & 0.000000 & 0.104995 \\
\mathrm{H} & 0.000000 & 0.979285 & -0.839961 \\
\mathrm{H} & 0.000000 & -0.979285 & -0.839961 \\
\end{array}$ \\
\hline
\end{tabular}

\begin{tabular}{|c|c|}
\hline $\begin{array}{l}\mathbf{O H}_{2} \\
\text { Energy }=-76.417914 \\
\text { Enthalpy }(0 \mathrm{~K})=-76.397123 \\
\text { Enthalpy }(298.15 \mathrm{~K})=-76.393343 \\
\text { Free Energy }=-76.414807\end{array}$ & $\begin{array}{l}\text { HF } \\
\text { Energy }=-100.424027 \\
\text { Enthalpy }(0 \mathrm{~K})=-100.414982 \\
\text { Enthalpy }(298.15 \mathrm{~K})=-100.411677 \\
\text { Free Energy }=-100.431410\end{array}$ \\
\hline $\begin{array}{llll}\mathrm{O} & 0.000000 & 0.000000 & 0.121147\end{array}$ & $\begin{array}{llll}\mathrm{H} & 0.000000 & 0.000000 & -0.839743\end{array}$ \\
\hline $\begin{array}{lll}0.000000 & 0.762472 & -0.484587\end{array}$ & $\begin{array}{cccc}\mathrm{F} & 0.000000 & 0.000000 & 0.093305\end{array}$ \\
\hline $\begin{array}{llll}\mathrm{H} & 0.000000 & -0.762472 & -0.484587\end{array}$ & \\
\hline
\end{tabular}

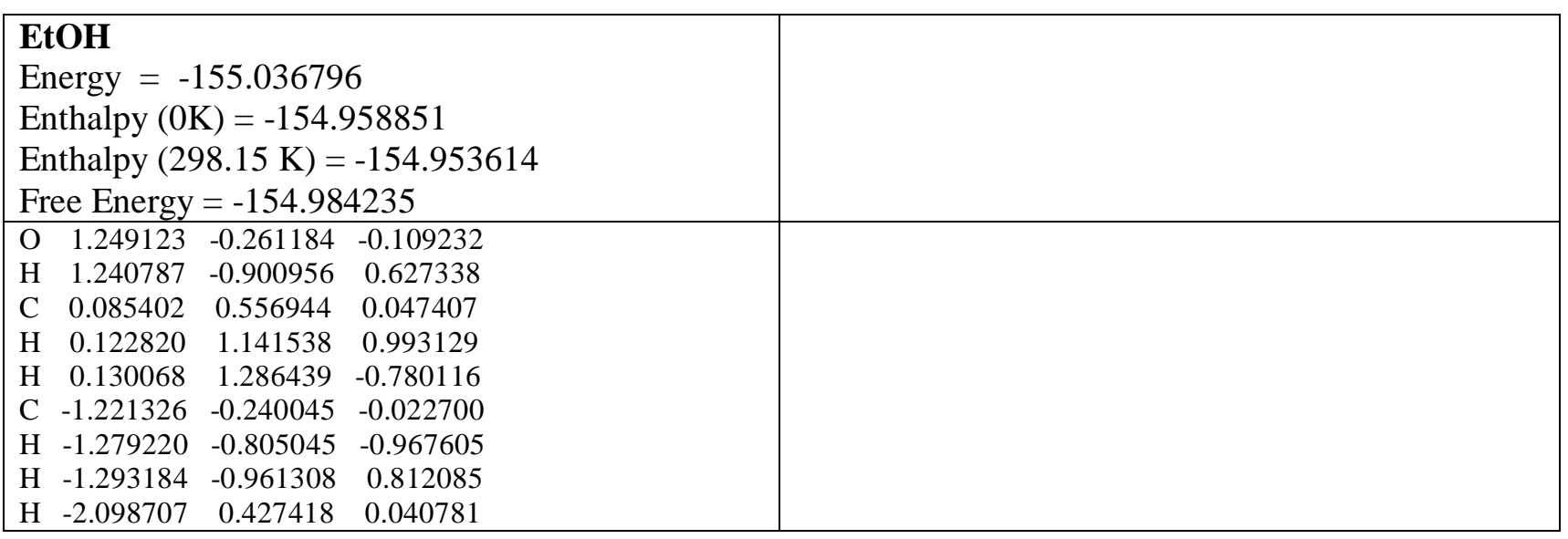

\begin{tabular}{|c|c|}
\hline $\begin{array}{l}\mathbf{C H}_{\mathbf{4}}, \\
\text { Energy = }-40.516189 \\
\text { Enthalpy }(0 \mathrm{~K})=-40.472289 \\
\text { Enthalpy }(298.15 \mathrm{~K})=-40.468471 \\
\text { Free Energy }=-40.491323\end{array}$ & $\begin{array}{l}\mathrm{SiH}_{\mathbf{4}}, \\
\text { Energy }=-6.289515 \\
\text { Enthalpy }(0 \mathrm{~K})=-6.259531 \\
\text { Enthalpy }(298.15 \mathrm{~K})=-6.255482 \\
\text { Free Energy }=-6.280479\end{array}$ \\
\hline $\begin{array}{llll}\mathrm{C} & 0.000000 & 0.000000 & 0.000032\end{array}$ & $\begin{array}{llll}\mathrm{Si} & 0.000000 & 0.000000 & 0.000018\end{array}$ \\
\hline $\begin{array}{llll}\mathrm{H} & 0.898011 & -0.000189 & -0.635046\end{array}$ & $\begin{array}{llll}\mathrm{H} & 0.000000 & 1.224786 & 0.866041\end{array}$ \\
\hline $\begin{array}{llll}\mathrm{H} & 0.000000 & 0.898104 & 0.634952\end{array}$ & $0.000339-0.866171$ \\
\hline $\begin{array}{llll}\mathrm{H} & 0.000000 & -0.898104 & 0.634952\end{array}$ & $\begin{array}{llll}\mathrm{H} & -1.224832 & -0.000339 & -0.866171\end{array}$ \\
\hline $\begin{array}{llll}\mathrm{H} & -0.898011 & 0.000189 & -0.635046\end{array}$ & $\begin{array}{llll}\mathrm{H} & 0.000000 & -1.224786 & 0.866041\end{array}$ \\
\hline
\end{tabular}

\begin{tabular}{|c|c|}
\hline $\begin{array}{l}\mathbf{P H}_{\mathbf{3}}, \\
\text { Energy }=-8.360816 \\
\text { Enthalpy }(0 \mathrm{~K})=-8.337770 \\
\text { Enthalpy }(298.15 \mathrm{~K})=-8.333906 \\
\text { Free Energy }=-8.358858\end{array}$ & $\begin{array}{l}\text { HCl, } \\
\text { Energy }=-15.628710 \\
\text { Enthalpy }(0 \mathrm{~K})=-15.622231 \\
\text { Enthalpy }(298.15 \mathrm{~K})=-15.618926 \\
\text { Free Energy }=-15.640140\end{array}$ \\
\hline $\begin{array}{llll}P & -0.000257 & 0.000119 & -0.133749\end{array}$ & $\begin{array}{llll}\mathrm{H} & 0.000000 & 0.000000 & -1.228630\end{array}$ \\
\hline $\begin{array}{llll}\mathrm{H} & 1.202339 & -0.043103 & 0.666997\end{array}$ & $\begin{array}{llll}\mathrm{Cl} & 0.000000 & 0.000000 & 0.072272\end{array}$ \\
\hline $\begin{array}{llll}\mathrm{H} & -0.636064 & -1.019168 & 0.668980\end{array}$ & \\
\hline $\begin{array}{llll}\text { H } & -0.562413 & 1.060484 & 0.670253\end{array}$ & \\
\hline
\end{tabular}


